\title{
$\left[\left({ }^{\mathrm{i}} \mathrm{Pr}_{3} \mathbf{P}\right)_{6} \mathrm{Rh}_{6} \mathrm{H}_{12}\right]^{2+}$ : A High-Hydride Content Octahedron that Bridges the Gap Between Late and Early-Transition Metal Clusters.
}

\author{
Michael J. Ingleson, Mary F. Mahon, Paul R. Raithby and Andrew S. Weller* \\ Department of Chemistry, University of Bath, Bath, UK, BA2 7AY
}

\section{Supporting Information}

Experimental Details for $\mathbf{1 a}$ and $\mathbf{1 b}$ (including mass spec data) Ball and stick diagram of the gross structural features of $\left.\left[{ }^{\mathrm{i}} \mathrm{Pr}\right)_{3} \mathrm{PH}\right]\left[\mathrm{CB}_{11} \mathrm{Me}_{11} \mathrm{H}\right]$. Experimental details for the in situ observation of dihydrogen species $\mathrm{X}$-ray data collection, refinement details and bond lengths and angles for $\mathbf{1 a}$ and $\mathbf{1 b}$ Details of HYDEX analysis 


\section{Experimental}

General. All manipulations were performed under an inert atmosphere of argon, using standard Schlenk-line and glove box techniques. Glassware was dried in an oven at $130^{\circ} \mathrm{C}$ overnight and flamed with a blowtorch, under vacuum, three times before use. $\mathrm{CH}_{2} \mathrm{Cl}_{2}, \mathrm{C}_{6} \mathrm{H}_{5} \mathrm{~F}$ and pentane were distilled from $\mathrm{CaH}_{2}$ and pentane from sodium. $\mathrm{CD}_{2} \mathrm{Cl}_{2}$ was distilled under vacuum from $\mathrm{CaH}_{2}$. Microanalyses were by Mr. Alan Carver (University of Bath Microanalytical Service). Ag[closo$1-\mathrm{H}-\mathrm{CB}_{11} \mathrm{Me}_{11}{ }^{(1)}$ was prepared using literature procedures. $\left[\left({ }^{\mathrm{i}} \mathrm{Pr}_{3} \mathrm{P}\right)_{2} \mathrm{Rh}(\mathrm{nbd})\right]\left(\mathrm{CB}_{11} \mathrm{Me}_{11} \mathrm{H}\right)$ or $\left[\left({ }^{\mathrm{i}} \mathrm{Pr}_{3} \mathrm{P}\right)_{2} \mathrm{Rh}(\mathrm{nbd})\right]\left[\mathrm{B}\left(\mathrm{Ar}_{\mathrm{F}}\right)_{4}\right]$ were prepared by an adaptation of the published route using $\mathrm{Ag}\left[\right.$ closo-1-H-CB $\left.{ }_{11} \mathrm{Me}_{11}\right], \mathrm{K}\left[\mathrm{B}\left(\mathrm{Ar}_{\mathrm{F}}\right)_{4}\right]{ }^{(2)}$ and ${ }^{\mathrm{i}} \mathrm{Pr}_{3} \mathrm{P} .{ }^{(3)}$

(1) Prepared by an adaptation of the method given in King, B. T.; Janousek, Z.; Gruner, B.; Trammell, M.; Noll, B. C.; Michl, J. J. Am. Chem. Soc. 1996, 118, 3313]; J. Michl, personal communication, 2002

(2) Buschmann, W. E.; Miller, J. S. Inorg. Synth. 2002, 33, 85

(3) Rifat, A.; Laing, V. E.; Kocoik-Köhn, G.; Mahon, M. F.; Ruggiero, G. D. and Weller, A. S. J. Organomet. Chem. 2003, 680, 127

NMR spectroscopy. ${ }^{1} \mathrm{H},{ }^{11} \mathrm{~B}$ and ${ }^{31} \mathrm{P}$ NMR spectra were recorded on a Bruker Advance $300 \mathrm{MHz}$ or Varian $400 \mathrm{MHz}$ (Bath) FT-NMR spectrometers. Residual protio solvent was used as reference for ${ }^{1} \mathrm{H}$ NMR spectra $\left(\mathrm{CD}_{2} \mathrm{Cl}_{2}: \square=5.33\right) .{ }^{11} \mathrm{~B}$ and ${ }^{31} \mathrm{P}$ NMR spectra were referenced against $\mathrm{BF}_{3} . \mathrm{OEt}_{2}$ (external) and $85 \% \mathrm{H}_{3} \mathrm{PO}_{4}$ (external) respectively. Coupling constants are quoted in Hertz.

\section{$\left[\mathrm{Rh}_{6}\left({ }^{\mathrm{i}} \operatorname{Pr}_{3} \mathrm{P}\right)_{6} \mathrm{H}_{12}\right]\left[\mathrm{CB}_{11} \mathrm{Me}_{11} \mathrm{H}\right]_{2} \mathbf{1 a}$}

A Young's tube was charged with $\left(0.030 \mathrm{~g}, 3.7 \times 10^{-5} \mathrm{~mol}\right)\left[\left({ }^{\mathrm{i}} \mathrm{Pr}_{3} \mathrm{P}\right)_{2} \mathrm{Rh}(\mathrm{nbd})\left(\mathrm{CB}_{11} \mathrm{Me}_{11} \mathrm{H}\right)\right]$ and dissolved in $\mathrm{C}_{6} \mathrm{H}_{5} \mathrm{~F}(5 \mathrm{ml})$. This was fully degassed by three freeze-pump-thaw cycles then backfilled immediately with $\mathrm{H}_{2}$ at 4 atmospheres pressure (77K warming to room temperature) resulting in a colour change from orange to yellow. Stirring for four days at $40^{\circ} \mathrm{C}$ yielded a further gradual darkening of the solution to dark brown. NMR analysis of the reaction mixture by ${ }^{31} \mathrm{P}\left\{{ }^{1} \mathrm{H}\right\}$ NMR spectroscopy showed three products formed in an approximate 1:1:1 ratio based on phosphorus. Two are identified as $\mathbf{1 a}$ and $\left[\mathrm{HP}^{\mathrm{i}} \mathrm{Pr}_{3}\right]\left[\mathrm{CB}_{11} \mathrm{Me}_{11} \mathrm{H}\right]$. The third component shows no hydride signal in the ${ }^{1} \mathrm{H}$ NMR spectrum and a single doublet in the ${ }^{31} \mathrm{P}\left\{{ }^{1} \mathrm{H}\right\}$ NMR spectrum $[\square$ $60.4 \mathrm{ppm}, J(\mathrm{RhP}) 110 \mathrm{~Hz}]$ under slight pressure of $\mathrm{H}_{2}$. Attempts to identify this third component have so far failed, but studies are ongoing. The solvent was removed in-vacuo and re-dissolving the residue in $\mathrm{CH}_{2} \mathrm{Cl}_{2}$ and layering with pentane yielded black $\left[\mathrm{Rh}_{6}\left({ }^{i} \mathrm{Pr}_{3} \mathrm{P}\right)_{6} \mathrm{H}_{12}\right]\left[\mathrm{CB}_{11} \mathrm{Me}_{11} \mathrm{H}\right]_{2} \mathbf{1 a}$ and colourless crystals of co-product $\left[\mathrm{HP}^{\mathrm{i}} \mathrm{Pr}_{3}\right]\left[\mathrm{CB}_{11} \mathrm{Me}_{11} \mathrm{H}\right]$. Yield $\left[\mathrm{Rh}_{6}\left({ }^{\mathrm{i}} \mathrm{Pr}_{3} \mathrm{P}\right)_{6} \mathrm{H}_{12}\right]\left[\mathrm{CB}_{11} \mathrm{Me}_{11} \mathrm{H}\right]_{2}$ $(0.016 \mathrm{~g}, 20 \%$ based on $\mathrm{Rh})$. $1 \mathbf{b}$ was prepared in an identical manner starting from $\left[\left({ }^{\mathrm{i}} \mathrm{Pr}_{3} \mathrm{P}\right)_{2} \mathrm{Rh}(\mathrm{nbd})\right]\left[\mathrm{B}\left(\mathrm{Ar}_{\mathrm{F}}\right)_{4}\right]$.

NMR Data (all in $\mathrm{CD}_{2} \mathrm{Cl}_{2}$ at 298K). $\left[\operatorname{Rh}_{6}\left({ }^{\mathrm{i}} \operatorname{Pr}_{3} \mathrm{P}\right)_{6} \mathrm{H}_{12}\right]\left[\mathrm{CB}_{11} \mathrm{Me}_{11} \mathrm{H}\right]_{2}$ 1a: ${ }^{1} \mathrm{H}(400 \mathrm{MHz}, 5$ sec delay between acquisitions, 1000 scans) $2.31(\mathrm{br} \mathrm{m} 18 \mathrm{H}), 1.28(\mathrm{~m}, 108 \mathrm{H}), 1.16\left(\mathrm{~s}, 2 \mathrm{H}\right.$ cage $\left._{\mathrm{C}-\mathrm{H}}\right),-0.17(\mathrm{~s}, 30 \mathrm{H}),-0.43(\mathrm{~s}, 30 \mathrm{H}),-$ $0.54(\mathrm{~s}, 6 \mathrm{H}),-21.44\left(\mathrm{br} \mathrm{s}, 12 \mathrm{H}\right.$, relative to ${ }^{\mathrm{i}} \mathrm{Pr}_{3}$ groups, integration consistent over 4 independently synthesized samples). ${ }^{11} \mathbf{B}$ (96 MHz) 0.17 (s, 1B), -7.89 (s, 5B), -11.14 (s, 5B). ${ }^{31} \mathbf{P}\left\{{ }^{1} \mathbf{H}\right\}$ (121MHz) $110.5(\mathrm{~d}, 140 \mathrm{~Hz})$.

$\mathrm{C}_{78} \mathrm{H}_{206} \mathrm{~B}_{22} \mathrm{P}_{6} \mathrm{Rh}_{6}$ requires $\% \mathrm{C} 42.9 \% \mathrm{H} \mathrm{9.43,} \mathrm{found} \% \mathrm{C} 43.6 \% \mathrm{H} 8.86$ 
$\left[\operatorname{Rh}_{6}\left({ }^{\mathrm{i}} \mathrm{Pr}_{3} \mathrm{P}\right)_{6} \mathrm{H}_{12}\right]\left[\mathrm{BAr}_{\mathrm{F}}\right]_{2} \mathrm{lb}:{ }^{1} \mathrm{H}(400 \mathrm{MHz}, 400 \mathrm{MHz}, 5$ sec delay between acquisitions, 1000 scans) $7.63\left(\mathrm{~s}, 16 \mathrm{H}\right.$, ortho arene) $7.47\left(\mathrm{~s}, 8 \mathrm{H}\right.$, para arene), $2.35\left(\mathrm{~m}, 18 \mathrm{H},{ }^{\mathrm{I}} \mathrm{Pr}\right.$ bridgehead) $1.26(\mathrm{~m}$, $\left.108 \mathrm{H}, \mathrm{CH}_{3}\right),-21.40\left(\mathrm{br}, \mathrm{s}, 12 \mathrm{H}\right.$, relative to ${ }^{\mathrm{i}} \mathrm{Pr}_{3}$ groups $) .{ }^{11} \mathbf{B}(128 \mathrm{MHz})-5.9(\mathrm{~s}){ }^{31} \mathbf{P}\left\{{ }^{1} \mathbf{H}\right\}$ : $(121 \mathrm{MHz}) 110.5(\mathrm{~d}, 140 \mathrm{~Hz})$.

$\mathrm{C}_{82} \mathrm{H}_{78} \mathrm{~B}_{2} \mathrm{~F}_{48} \mathrm{P}_{6} \mathrm{Rh}_{6}$ requires \%C 35.2\% \%H 2.81\%, found \%C $34.9 \% \mathrm{H} 3.29$

Mass Spec (FAB+, NOBA matrix): $1590.3\left[\mathrm{Rh}_{6}\left({ }^{i} \mathrm{Pr}_{3} \mathrm{P}\right)_{6} \mathrm{H}_{12}\right]^{+}$. showing the correct isotope pattern for $\mathrm{Rh}_{6} \mathrm{C}_{54} \mathrm{H}_{138} \mathrm{P}_{6}$ (see below)


Observed for $1 \mathbf{a}$ 
NMR data for the $\left[{ }^{\mathrm{i}} \mathrm{Pr}_{3} \mathrm{PH}\right]^{+}$cation are consistent with that reported by Torres et al. (J. Am. Chem. Soc. 1999, 121, 10632-10633) for $\left[{ }^{\mathrm{i}} \mathrm{Pr}_{3} \mathrm{PH}\right] \mathrm{BF}_{4}$. The gross structure was confirmed by a single crystal X-ray diffraction study, but due the poor quality of crystals the data refinement was poor $\left(w \mathrm{R}_{1}=10.6 \%\right)$ and thus not reported in full here. Figure $\mathrm{S} 1$ shows a ball and stick plot for $\left[\mathrm{HP}^{\mathrm{i}} \mathrm{Pr}_{3}\right]\left[\mathrm{CB}_{11} \mathrm{Me}_{11} \mathrm{H}\right]$.
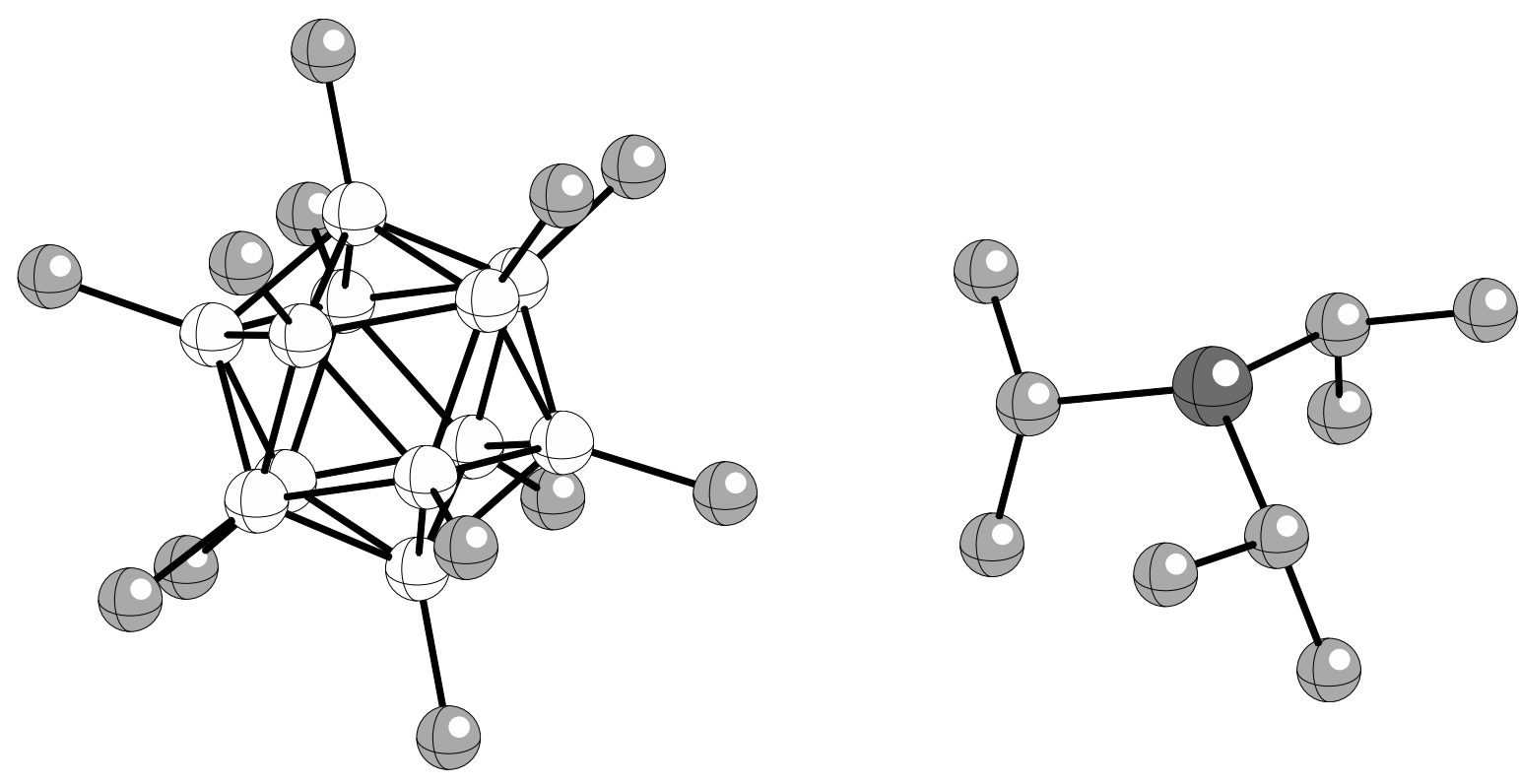

Figure S1: Ball and stick diagram of the solid-state structure of $\left[\left({ }^{\mathrm{i}} \mathrm{Pr}\right)_{3} \mathrm{PH}\right]\left[\mathrm{CB}_{11} \mathrm{Me}{ }_{11} \mathrm{H}\right]$. The hydrogen atoms are not shown. The cage anion is disordered and the cage carbon atom was not located. 
$\left.\left[{ }^{i} \operatorname{Pr}_{3} P\right)_{2} \mathbf{R h}\left(\mathrm{H}_{2}\right)_{\mathrm{X}} \mathrm{H}_{2}\right][Y]:\left(\mathrm{x}=1\right.$ or 2 and $\mathrm{Y}=1-\mathrm{H}-\mathrm{CB}_{11}\left(\mathrm{CH}_{3}\right)_{11}$ or $\left.\mathrm{B}\left(\mathrm{Ar}_{\mathrm{F}}\right)_{4}\right)$

A typical experiment consisted of charging a Young's NMR tube with $0.015 \mathrm{~g}$ of $\left[\left({ }^{\mathrm{i}} \mathrm{Pr}_{3} \mathrm{P}\right)_{2} \mathrm{RhNBD}\right][\mathrm{Y}]$, addition of $0.3 \mathrm{ml}$ of $\mathrm{CD}_{2} \mathrm{Cl}_{2}$ generated an orange solution. The sample was freeze/pumped/thawed three times before being backfilled with $\mathrm{H}_{2}$ at $77 \mathrm{~K}\left(\sim 4 \mathrm{~atm} \mathrm{H}_{2}\right.$ at $\left.298 \mathrm{~K}\right)$. On thawing, the solution rapidly changes colour from orange to pale yellow. Attempts to isolate solid material repeatedly failed. ${ }^{1} \mathrm{H} \mathrm{NMR}\left(400 \mathrm{MHz}, \mathrm{CD}_{2} \mathrm{Cl}_{2}, 298 \mathrm{~K}\right.$ for $\left.\mathrm{Y}=\mathrm{B}\left(\mathrm{Ar}_{\mathrm{F}}\right)_{4}\right) \square 7.73$ (s, $8 \mathrm{H}$, ortho arene), 7.57 (s, $4 \mathrm{H}$, para arene), $2.31\left(\mathrm{~m}, 6 \mathrm{H},{ }^{\mathrm{i}} \mathrm{Pr}\right.$ bridgehead), 1.25 [dd $36 \mathrm{H}, \mathrm{CH}_{3}, 7$, $15 \mathrm{~Hz}],-8.62$ (v. br $3.6 \mathrm{H}$ relative to ${ }^{\mathrm{i}} \operatorname{Pr}$ protons, $\left.\left(\mathrm{H}_{2}\right) / \mathrm{H}, \mathrm{T}_{1} 106 \mathrm{~ms}\right) .{ }^{31} \mathrm{P}\left\{{ }^{1} \mathrm{H}\right\}$ NMR $(162 \mathrm{MHz}$, $\left.\mathrm{CD}_{2} \mathrm{Cl}_{2}, 298 \mathrm{~K}\right) \square 60.4$ [d, $\left.J(\mathrm{RhP}) 107 \mathrm{~Hz}\right] .{ }^{11} \mathrm{~B}$ NMR $\left(128 \mathrm{MHz}, \mathrm{CD}_{2} \mathrm{Cl}_{2}, 298 \mathrm{~K}\right) \square-5.9(\mathrm{~s})$.

\section{$\left[\left({ }^{\mathrm{i}} \mathbf{P r}_{3} \mathbf{P}\right)_{2} \mathbf{R h}\left(\mathbf{H}_{2}\right) \mathbf{H}_{2}\right]\left[\mathrm{BAr}_{\mathrm{F}}\right]$ :}

${ }^{1} \mathrm{H}$ NMR (400MHz, $\left.\mathrm{CD}_{2} \mathrm{Cl}_{2}, \mathbf{1 9 0 K}\right) \square 7.61$ (s, 8H, ortho arene), 7.46 (s, $4 \mathrm{H}$, para arene), 2.08, (m, $6 \mathrm{H},{ }^{\mathrm{I}} \mathrm{Pr}$ bridgehead), $1.02\left(\mathrm{~m}, 36 \mathrm{H}, \mathrm{CH}_{3}\right),-0.28$ (br $\left.2 \mathrm{H}\left(\square^{2}-\mathrm{H}_{2}\right), \mathrm{T}_{1} 94 \mathrm{~ms}\right),-12.66$ (s br, $1 \mathrm{H}, \mathrm{T}_{1}$ $238 \mathrm{~ms}),-22.42$ (s br, $\left.1 \mathrm{H}, \mathrm{T}_{1} 234\right) .{ }^{31} \mathrm{P}\left\{{ }^{1} \mathrm{H}\right\}\left(162 \mathrm{MHz}, \mathrm{CD}_{2} \mathrm{Cl}_{2}, 190 \mathrm{~K}\right) 62.1[\mathrm{~d}, J(\mathrm{RhP}) 100 \mathrm{~Hz}]$

\section{$\left[\left({ }^{\mathrm{i}} \mathrm{Pr}_{3} \mathbf{P}\right)_{2} \mathbf{R h}\left(\mathbf{H}_{2}\right)_{2} \mathbf{H}_{2}\right]\left[\mathbf{B}\left(\mathrm{Ar}_{\mathrm{F}}\right)_{4}\right]$ :}

${ }^{1} \mathrm{H}$ NMR (400MHz, $\left.\mathrm{CD}_{2} \mathrm{Cl}_{2}, 190 \mathrm{~K}\right) \square 7.61$ (s, $8 \mathrm{H}$, ortho arene), 7.46 (s, 4H, para arene), 2.08, (m, $6 \mathrm{H},{ }^{\mathrm{I}} \mathrm{Pr}$ bridgehead), $1.02\left(\mathrm{~m}, 36 \mathrm{H}, \mathrm{CH}_{3}\right),-2.06$ (v. br $4 \mathrm{H}\left(\square^{2}-\mathrm{H}_{2}\right), \mathrm{T}_{1} 43 \mathrm{~ms}$ ), -14.23 (s br, $2 \mathrm{H}, \mathrm{T}_{1}$ $360 \mathrm{~ms}),{ }^{31} \mathrm{P}\left\{{ }^{1} \mathrm{H}\right\}\left(162 \mathrm{MHz}, \mathrm{CD}_{2} \mathrm{Cl}_{2}, 190 \mathrm{~K}\right) \square 68.4[\mathrm{~d}, J(\mathrm{RhP}) 92 \mathrm{~Hz}]$.

At $190 \mathrm{~K}$ the ratio $\left.\left[\left({ }^{\mathrm{i}} \mathrm{Pr}_{3} \mathrm{P}\right)_{2} \mathrm{Rh}\left(\mathrm{H}_{2}\right) \mathrm{H}_{2}\right]\left[\mathrm{BAr} \mathrm{F}_{\mathrm{F}}\right]:\left[{ }^{\mathrm{i}} \mathrm{Pr}_{3} \mathrm{P}\right)_{2} \mathrm{Rh}\left(\mathrm{H}_{2}\right)_{2} \mathrm{H}_{2}\right]\left[\mathrm{BAr} \mathrm{F}_{\mathrm{F}}\right]$ is $4: 1$. Assignment of the hydrides in each complex comes from $\mathrm{T}_{1}$ measurements* and comparison of chemical shifts with the previously reported complexes $\left[\left(\mathrm{R}_{3} \mathrm{P}\right)_{2} \operatorname{Ir}\left(\mathrm{H}_{2}\right)_{\mathrm{x}} \mathrm{H}_{2}\right]\left[\mathrm{B}\left(\mathrm{Ar}_{\mathrm{F}}\right)_{4}\right](\mathrm{x}=1,2){ }^{(4)}$

* $\mathrm{T}_{1}$ measurements were made at $190 \mathrm{~K}$ using the software provided with a Bruker Advance 400 spectrometer using the standard inversion-recovery-delay method $\left(180^{\circ} \square \square 90^{\circ}\right)$ method. The calculation of relaxation times was made using a nonlinear three-parameter fitting routine. In each experiment a waiting period of 5 times longer than the expected relaxation time and 11 variable delays were used.

(4) (a) Cooper, A. C.; Caulton, K. G. Inorg Chem. 1998, 37, 5938. (b) Cooper, A. C.; Eisenstein, O.; Caulton, K. G. New J. Chem. 1998, 307. (c) Crabtree, R. H.; Lavin, M. Chem. Commun. 1985, 1661 


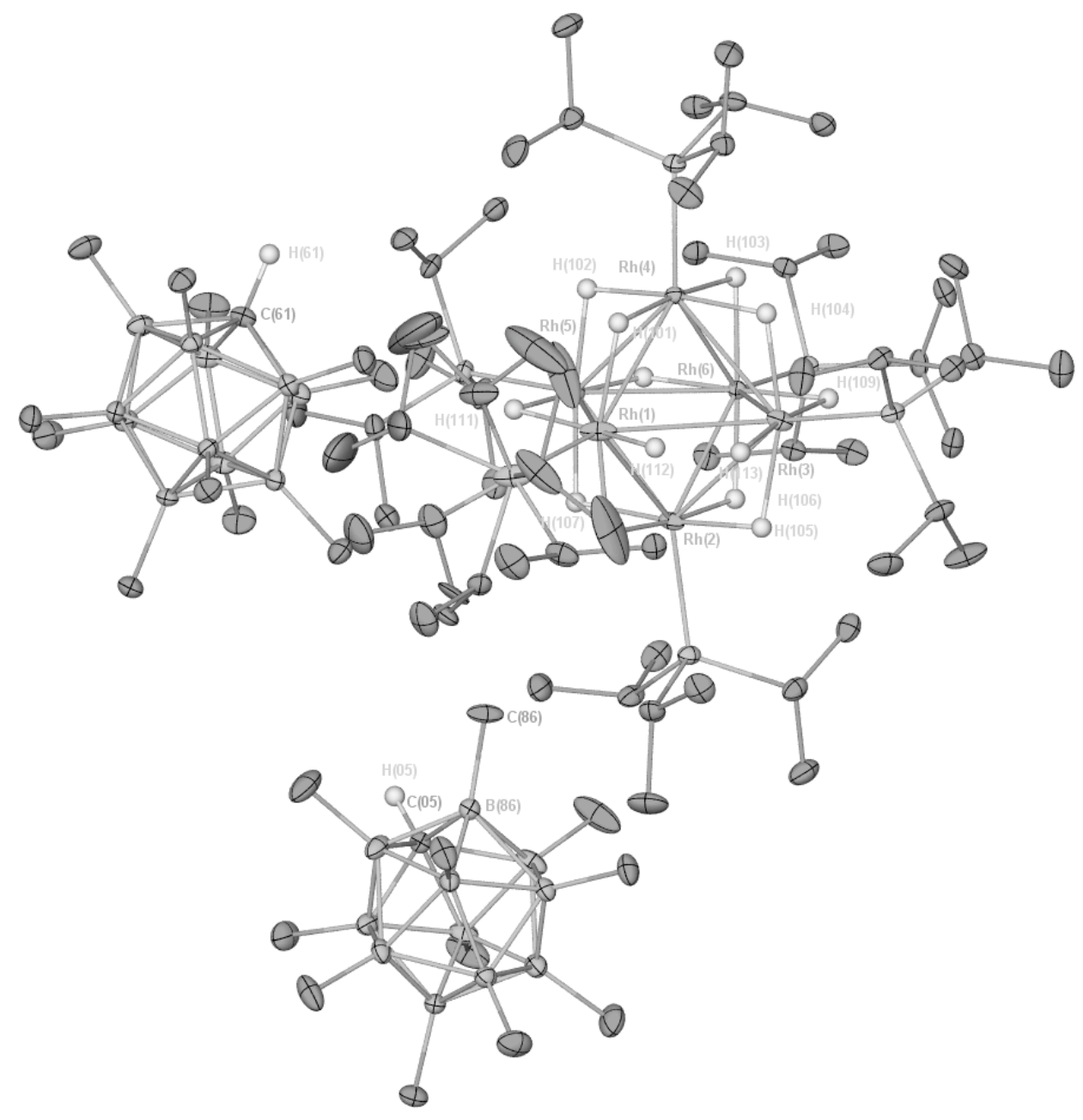

Figure S2: $\left[\mathrm{Rh}_{6}\left({ }^{\mathrm{i}} \mathrm{Pr}_{3} \mathrm{P}\right)_{6} \mathrm{H}_{12}\right]\left[\mathrm{CB}_{11} \mathrm{Me}_{11} \mathrm{H}\right]_{2}$, 1a, showing atom labelling scheme and the disorder associated with $\mathrm{P} 1$ and $\mathrm{H} 12 / \mathrm{H} 13$ for $1 \mathrm{a}$. Thermal ellipsoids are shown at the $50 \%$ probability level. The asymmetric unit in this structure consists of 1 cationic cluster, 2 anionic carboranes, and 3 area of diffuse solvent. Equal disorder of the isopropyl groups associated with the phosphine P1 in the cluster was readily modelled between the atoms with labels without suffixes and those labelled with suffix 'A'. The $\mathrm{C} \square \mathrm{H}$ moiety in one of the anions was over 2 sites. In particular C05, B86 and C86 (and pendant hydrogens) share occupancy with C06, B85 and C85 respectively. The solvent regions were diffuse and ultimately 'mopped】up' using partial carbons with site occupancy (C901】909). At this stage of the convergence the bridging hydride atoms at the cluster core were located, without prejudice, in a difference Fourier electron density map using data up to a $2 \square$ maximum of $60^{\circ}$. All hydrides were treated as having full occupancy, with the exceptions of $\mathrm{H} 112$ and H113. Bridging distances from each hydride to the core pair of bonded rhodium atoms were constrained to be similar during the final least squares refinement. The metal $\square$ hydride distances involving H112 and H113 were also constrained to be similar to each other. This course of action was chosen largely to aid the refinement giving the shortcomings of $\mathrm{X} \square$ ray data in the determination of these small species. Assignment of the site occupancy concerning H112 and H113 was made on chemical grounds as opposed to anything structural. That is to say, making H112 and H113 both full occupancy would give the cluster 13 hydrides and thus be paramagnetic, contrary to the diamagnetism observe in the NMR spectra. Treatment of these hydrides as having full occupancies did not have a decisive effect of the refinement. 


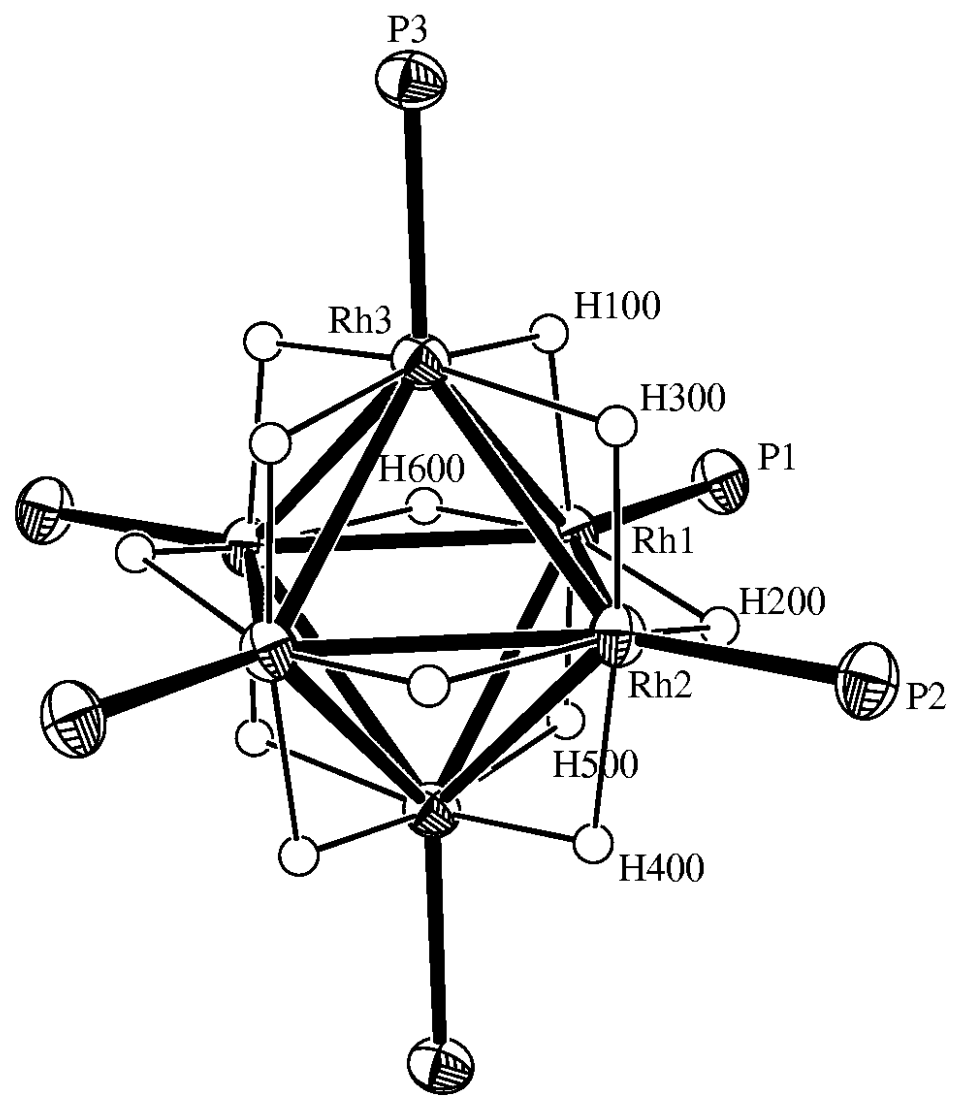

Figure S3: $\left[\mathrm{Rh}_{6}\left({ }^{\mathrm{i}} \mathrm{Pr}_{3} \mathrm{P}\right)_{6} \mathrm{H}_{12}\right]\left[\mathrm{B}\left(\mathrm{Ar}_{\mathrm{F}}\right)_{4}\right]_{2}, \mathbf{1}$ b, showing atom labelling scheme. Only dicationic portion shown, with $\left[\mathrm{B}\left(\mathrm{Ar}_{\mathrm{F}}\right)_{4}\right]$ anions and one molecule of solvent $\left(\mathrm{C}_{6} \mathrm{H}_{5} \mathrm{~F}\right)$ not shown. ${ }^{\mathrm{I}} \mathrm{Pr}$ groups on the cluster phosphine ligands have also been omitted for clarity. The molecule is centrosymetric, with half of the octahedron (and one anion) generated crystallographically. Thermal ellipsoids are shown at the $50 \%$ probability level. The $\left[\mathrm{Rh}_{6}\right]^{2+}$ dication sits on a center of inversion. All six bridging hydrogens in the asymmetric unit could be located in the Fourier map due to the good quality of the high angle $\left(60^{\circ}\right)$ data. They have a site occupation factor of 1 and could be refined isotropically. In the unique anion four of the eight attached $\mathrm{CF}_{3}$ groups show rotational disorder. The complex crystallizes with a half solvent molecule of fluorobenzene in the asymmetrical unit. The Fluorine in this solvent molecule is disordered in the ratio of 1:1 and due to the molecule sitting on a center of inversion it occupies four different positions. 
Table S1. Crystal data and structure refinement for $\mathbf{1 a .}$

\begin{tabular}{|c|c|}
\hline Identification code & Compound 1 \\
\hline Empirical formula & C82.50 H206 B22 P6 Rh6 \\
\hline Formula weight & 2239.57 \\
\hline Temperature & $150(2) \mathrm{K}$ \\
\hline Wavelength & $0.71073 \AA$ \\
\hline Crystal system & Monoclinic \\
\hline Space group & $\mathrm{P} 2_{1} / \mathrm{n}$ \\
\hline \multirow[t]{3}{*}{ Unit cell dimensions } & $a=21.50300(10) \AA \square=90^{\circ}$ \\
\hline & $\mathrm{b}=25.48900(10) \AA \mathrm{C}=103.30^{\circ}$ \\
\hline & $c=21.82500(10) \AA \square=90^{\circ}$ \\
\hline Volume & $11641.32(9) \AA^{3}$ \\
\hline Z & 4 \\
\hline Density (calculated) & $1.278 \mathrm{Mg} / \mathrm{m}^{3}$ \\
\hline Absorption coefficient & $0.949 \mathrm{~mm}^{-1}$ \\
\hline $\mathrm{F}(000)$ & 4684 \\
\hline Crystal size & $0.43 \times 0.15 \times 0.13 \mathrm{~mm}$ \\
\hline Theta range for data collection & 3.60 to $30.08^{\circ}$ \\
\hline Index ranges & $-30<=\mathrm{h}<=29 ;-35<=\mathrm{k}<=35 ;-30<=\mathrm{l}<=30$ \\
\hline Reflections collected & 205546 \\
\hline Independent reflections & $33956[R($ int $)=0.0662]$ \\
\hline Reflections observed (>2 $\square$ ) & 23910 \\
\hline Data Completeness & 0.993 \\
\hline Absorption correction & Semi-empirical from equivalents \\
\hline Max. and min. transmission & 0.92 and 0.86 \\
\hline Refinement method & Full-matrix least-squares on $\mathrm{F}^{2}$ \\
\hline Data / restraints / parameters & $33956 / 12$ / 1251 \\
\hline Goodness-of-fit on $\mathrm{F}^{2}$ & 1.034 \\
\hline Final $R$ indices $[I>2 \square(I)]$ & $R_{1}=0.0480 \quad w R_{2}=0.1007$ \\
\hline $\mathrm{R}$ indices (all data) & $R_{1}=0.0832 w_{2}=0.1163$ \\
\hline Largest diff. peak and hole & 1.629 and $-2.201 \mathrm{e}^{-3}$ \\
\hline
\end{tabular}


Table S2. Atomic coordinates $\left(\times 10^{4}\right)$ and equivalent isotropic displacement parameters $\left(\AA^{2} \times 10^{3}\right)$ for $1 . U(e q)$ is defined as one third of the trace of the orthogonalized Uij tensor.

\begin{tabular}{|c|c|c|c|c|}
\hline Atom & $x$ & $y$ & $z$ & $\mathrm{U}(\mathrm{eq})$ \\
\hline $\mathrm{Rh}(1)$ & $5405(1)$ & $3006(1)$ & $3530(1)$ & $40(1)$ \\
\hline $\operatorname{Rh}(2)$ & $6631(1)$ & $3272(1)$ & $3420(1)$ & $28(1)$ \\
\hline $\operatorname{Rh}(3)$ & $6173(1)$ & $2298(1)$ & $2844(1)$ & 29(1) \\
\hline $\mathrm{Rh}(4)$ & $5966(1)$ & $2030(1)$ & 4020(1) & $22(1)$ \\
\hline $\mathrm{Rh}(5)$ & $6435(1)$ & 2952(1) & $4563(1)$ & $24(1)$ \\
\hline $\mathrm{Rh}(6)$ & $7144(1)$ & $2360(1)$ & $3932(1)$ & 21(1) \\
\hline $\mathrm{P}(1)$ & $4534(1)$ & $3514(1)$ & $3322(1)$ & 51(1) \\
\hline $\mathrm{P}(2)$ & 6998(1) & $3932(1)$ & 2911(1) & $28(1)$ \\
\hline$P(3)$ & 5961(1) & 1810(1) & 1962(1) & $26(1)$ \\
\hline$P(4)$ & $5513(1)$ & 1313(1) & $4345(1)$ & $26(1)$ \\
\hline$P(5)$ & $6606(1)$ & $3286(1)$ & $5541(1)$ & $28(1)$ \\
\hline$P(6)$ & $8130(1)$ & 2022(1) & 4205(1) & $23(1)$ \\
\hline $\mathrm{C}(1)$ & $4282(4)$ & $3720(3)$ & $2447(4)$ & $47(2)$ \\
\hline $\mathrm{C}(2)$ & $4413(4)$ & $3335(4)$ & $1940(4)$ & $36(2)$ \\
\hline $\mathrm{C}(3)$ & $3570(5)$ & $3908(4)$ & $2281(5)$ & $63(3)$ \\
\hline $\mathrm{C}(4)$ & $3872(4)$ & $3172(4)$ & $3502(6)$ & $68(3)$ \\
\hline $\mathrm{C}(05)$ & $3638(4)$ & $5735(3)$ & 1634(4) & $27(2)$ \\
\hline $\mathrm{C}(5)$ & $3638(12)$ & 2694(11) & $3131(16)$ & 115(11) \\
\hline $\mathrm{C}(06)$ & $3248(5)$ & $5329(4)$ & 1053(5) & $31(3)$ \\
\hline $\mathrm{C}(6)$ & $3948(6)$ & $3125(7)$ & $4260(8)$ & $70(4)$ \\
\hline $\mathrm{C}(7)$ & $4700(4)$ & 4071(4) & $3774(5)$ & $54(2)$ \\
\hline $\mathrm{C}(8)$ & $5184(14)$ & $4401(6)$ & $3616(15)$ & $48(5)$ \\
\hline $\mathrm{C}(9)$ & 4096(6) & $4385(5)$ & $3820(6)$ & $79(4)$ \\
\hline $\mathrm{C}(10)$ & $6323(2)$ & $4327(2)$ & $2451(2)$ & $38(1)$ \\
\hline$C(11)$ & $6506(3)$ & $4839(2)$ & $2168(3)$ & $67(2)$ \\
\hline $\mathrm{C}(12)$ & $5891(2)$ & $3997(2)$ & 1944(2) & $48(1)$ \\
\hline$C(13)$ & $7474(2)$ & $3734(2)$ & $2339(2)$ & $35(1)$ \\
\hline $\mathrm{C}(14)$ & $7855(2)$ & $3228(2)$ & $2514(2)$ & $40(1)$ \\
\hline$C(15)$ & $7915(2)$ & $4163(2)$ & $2179(2)$ & $51(1)$ \\
\hline$C(16)$ & $7503(2)$ & $4403(2)$ & $3455(2)$ & $38(1)$ \\
\hline $\mathrm{C}(17)$ & $8089(2)$ & $4132(2)$ & $3861(2)$ & $48(1)$ \\
\hline $\mathrm{C}(18)$ & $7115(2)$ & $4684(2)$ & $3864(2)$ & 49(1) \\
\hline$C(19)$ & $6071(2)$ & $2153(2)$ & 1252(2) & $38(1)$ \\
\hline $\mathrm{C}(20)$ & $6766(2)$ & $2330(2)$ & $1325(2)$ & $66(2)$ \\
\hline $\mathrm{C}(21)$ & $5618(2)$ & $2615(2)$ & 1070(2) & $54(1)$ \\
\hline $\mathrm{C}(22)$ & $5145(2)$ & 1523(2) & $1760(2)$ & $32(1)$ \\
\hline$C(23)$ & $4964(2)$ & $1290(2)$ & 1095(2) & $36(1)$ \\
\hline$C(24)$ & $4616(2)$ & 1889(2) & 1861(2) & $53(1)$ \\
\hline$C(25)$ & $6521(2)$ & $1243(2)$ & $2050(2)$ & $36(1)$ \\
\hline $\mathrm{C}(26)$ & $6355(2)$ & $833(2)$ & $2496(2)$ & $47(1)$ \\
\hline $\mathrm{C}(27)$ & $6613(2)$ & 973(2) & 1444(2) & $56(1)$ \\
\hline $\mathrm{C}(28)$ & $4917(2)$ & $990(1)$ & $3706(2)$ & $34(1)$ \\
\hline$C(29)$ & $4640(2)$ & $477(2)$ & $3898(2)$ & $48(1)$ \\
\hline$C(30)$ & $4388(2)$ & $1357(2)$ & $3373(2)$ & $52(1)$ \\
\hline$C(31)$ & $5171(2)$ & 1448(2) & $5035(2)$ & $35(1)$ \\
\hline$C(32)$ & $5033(2)$ & $970(2)$ & $5412(2)$ & $50(1)$ \\
\hline
\end{tabular}




\begin{tabular}{|c|c|c|c|c|}
\hline$C(33)$ & $4584(2)$ & 1804(2) & $4875(2)$ & $55(1)$ \\
\hline$C(34)$ & $6113(2)$ & 797(1) & $4635(2)$ & $35(1)$ \\
\hline$C(35)$ & $6624(2)$ & $994(2)$ & $5199(2)$ & $44(1)$ \\
\hline$C(36)$ & $6424(2)$ & $593(2)$ & 4117(2) & $43(1)$ \\
\hline $\mathrm{C}(37)$ & $7468(2)$ & $3384(2)$ & $5888(2)$ & $38(1)$ \\
\hline$C(38)$ & $7655(2)$ & $3451(2)$ & $6610(2)$ & $62(1)$ \\
\hline$C(39)$ & $7755(2)$ & $3823(2)$ & $5567(2)$ & $46(1)$ \\
\hline$C(40)$ & $6145(2)$ & $3893(2)$ & $5587(2)$ & $39(1)$ \\
\hline$C(41)$ & $6246(2)$ & $4326(2)$ & $5143(2)$ & $47(1)$ \\
\hline$C(42)$ & $6212(3)$ & $4116(2)$ & 6252(2) & $69(2)$ \\
\hline$C(43)$ & 6339(2) & $2839(2)$ & 6094(2) & $34(1)$ \\
\hline$C(44)$ & $6694(2)$ & $2314(2)$ & $6153(2)$ & 41(1) \\
\hline$C(45)$ & $5614(2)$ & $2756(2)$ & $5918(2)$ & $43(1)$ \\
\hline$C(46)$ & $8239(2)$ & 1597(1) & 4911(2) & $28(1)$ \\
\hline $\mathrm{C}(47)$ & $8897(2)$ & $1338(2)$ & $5120(2)$ & $43(1)$ \\
\hline$C(48)$ & $8044(2)$ & 1854(2) & $5469(2)$ & $37(1)$ \\
\hline$C(49)$ & $8727(2)$ & $2558(1)$ & $4318(2)$ & $30(1)$ \\
\hline$C(50)$ & 9423(2) & $2383(2)$ & $4366(2)$ & $44(1)$ \\
\hline$C(51)$ & $8688(2)$ & $2923(2)$ & $4859(2)$ & 40(1) \\
\hline$C(52)$ & $8355(2)$ & 1593(2) & $3611(2)$ & $32(1)$ \\
\hline $\mathrm{C}(53)$ & $8340(2)$ & 1883(2) & $2995(2)$ & $42(1)$ \\
\hline$C(54)$ & $7946(2)$ & 1096(2) & $3496(2)$ & 41(1) \\
\hline$C(61)$ & $983(2)$ & $3495(1)$ & $3547(2)$ & $32(1)$ \\
\hline$C(62)$ & $2102(2)$ & $3338(2)$ & 3061(3) & $57(1)$ \\
\hline$C(63)$ & $571(2)$ & $3231(2)$ & $2234(2)$ & $43(1)$ \\
\hline$C(64)$ & $-383(2)$ & $3452(2)$ & $3218(2)$ & $40(1)$ \\
\hline$C(65)$ & $543(2)$ & $3712(2)$ & $4664(2)$ & $50(1)$ \\
\hline$C(66)$ & $2090(2)$ & $3643(2)$ & 4572(2) & $60(1)$ \\
\hline $\mathrm{C}(67)$ & 1379(3) & 4888(2) & 4597(2) & $55(1)$ \\
\hline$C(68)$ & $-200(2)$ & $4769(2)$ & $3730(2)$ & $45(1)$ \\
\hline$C(69)$ & $-179(2)$ & $4463(2)$ & 2191(2) & $40(1)$ \\
\hline$C(70)$ & $1430(2)$ & $4378(2)$ & $2078(2)$ & $41(1)$ \\
\hline$C(71)$ & $958(2)$ & $5338(1)$ & $3031(2)$ & $40(1)$ \\
\hline $\mathrm{C}(81)$ & $4483(2)$ & $4966(2)$ & 1114(4) & $86(2)$ \\
\hline$C(82)$ & $3214(2)$ & $5051(2)$ & $-203(2)$ & $45(1)$ \\
\hline$C(83)$ & $4437(2)$ & $5983(2)$ & 13(3) & $61(1)$ \\
\hline$C(84)$ & 4915(2) & $6209(2)$ & 1645(3) & $68(2)$ \\
\hline$C(85)$ & $4075(6)$ & $5352(4)$ & $2287(5)$ & $72(3)$ \\
\hline$C(86)$ & $2948(4)$ & $4767(3)$ & $1333(4)$ & $42(2)$ \\
\hline$C(87)$ & 1942(2) & $5593(2)$ & $293(2)$ & $48(1)$ \\
\hline$C(88)$ & $2850(3)$ & $6374(2)$ & $-485(2)$ & $56(1)$ \\
\hline$C(89)$ & $3902(2)$ & $7106(2)$ & $663(2)$ & $42(1)$ \\
\hline$C(90)$ & $3652(3)$ & $6746(2)$ & $2134(2)$ & $55(1)$ \\
\hline$C(91)$ & $2453(3)$ & $5807(2)$ & 1897(2) & $68(2)$ \\
\hline$C(92)$ & $2364(2)$ & $6862(2)$ & $829(3)$ & $57(1)$ \\
\hline $\mathrm{C}(107)$ & $2377(2)$ & $4662(2)$ & $3555(2)$ & $50(1)$ \\
\hline $\mathrm{C}(901)$ & $-1735(12)$ & 1819(10) & 1010(11) & 163(8) \\
\hline $\mathrm{C}(902)$ & $-1733(7)$ & $2224(6)$ & $551(7)$ & 93(4) \\
\hline $\mathrm{C}(903)$ & $-1491(7)$ & $2563(6)$ & $1026(7)$ & $95(4)$ \\
\hline $\mathrm{C}(904)$ & $-975(5)$ & $2723(5)$ & 1414(5) & $58(2)$ \\
\hline $\mathrm{C}(905)$ & $-949(7)$ & $2444(6)$ & 1589(7) & 91(4) \\
\hline $\mathrm{C}(906)$ & $6038(6)$ & $5778(5)$ & $3138(6)$ & $71(3)$ \\
\hline $\mathrm{C}(907)$ & 6186(9) & $5947(8)$ & 3442(9) & 115(6) \\
\hline $\mathrm{C}(908)$ & $4365(5)$ & $4163(4)$ & $5577(5)$ & $60(3)$ \\
\hline
\end{tabular}




\begin{tabular}{|l|l|l|l|l|}
\hline$C(909)$ & $4512(10)$ & $4079(8)$ & $6084(10)$ & $135(6)$ \\
\hline$B(62)$ & $1573(2)$ & $3724(2)$ & $3206(2)$ & $34(1)$ \\
\hline$B(63)$ & $763(2)$ & $3664(2)$ & $2767(2)$ & $29(1)$ \\
\hline$B(64)$ & $258(2)$ & $3784(2)$ & $3292(2)$ & $27(1)$ \\
\hline$B(65)$ & $745(2)$ & $3921(2)$ & $4050(2)$ & $34(1)$ \\
\hline$B(66)$ & $1563(2)$ & $3882(2)$ & $4002(2)$ & $38(1)$ \\
\hline$B(67)$ & $1185(2)$ & $4502(2)$ & $4002(2)$ & $33(1)$ \\
\hline$B(68)$ & $372(2)$ & $4442(2)$ & $3562(2)$ & $29(1)$ \\
\hline$B(69)$ & $388(2)$ & $4281(2)$ & $2768(2)$ & $26(1)$ \\
\hline$B(70)$ & $1207(2)$ & $4241(2)$ & $2712(2)$ & $28(1)$ \\
\hline$B(71)$ & $1698(2)$ & $4380(2)$ & $3476(2)$ & $34(1)$ \\
\hline$B(72)$ & $964(2)$ & $4728(1)$ & $3203(2)$ & $27(1)$ \\
\hline$B(81)$ & $3976(2)$ & $5433(2)$ & $1004(2)$ & $39(1)$ \\
\hline$B(82)$ & $3323(2)$ & $5480(2)$ & $344(2)$ & $29(1)$ \\
\hline$B(83)$ & $3946(2)$ & $5957(2)$ & $465(2)$ & $33(1)$ \\
\hline$B(84)$ & $4197(2)$ & $6080(2)$ & $1282(2)$ & $34(1)$ \\
\hline$B(85)$ & $3786(5)$ & $5650(4)$ & $1624(5)$ & $32(2)$ \\
\hline$B(86)$ & $3141(6)$ & $5339(4)$ & $1120(5)$ & $27(3)$ \\
\hline$B(87)$ & $2662(2)$ & $5758(2)$ & $602(2)$ & $29(1)$ \\
\hline$B(88)$ & $3134(2)$ & $6156(2)$ & $215(2)$ & $29(1)$ \\
\hline$B(89)$ & $3673(2)$ & $6526(2)$ & $796(2)$ & $28(1)$ \\
\hline$B(90)$ & $3539(2)$ & $6357(2)$ & $1541(2)$ & $35(1)$ \\
\hline$B(91)$ & $2911(2)$ & $5880(2)$ & $1421(2)$ & $39(1)$ \\
\hline$B(92)$ & $2879(2)$ & $6405(2)$ & $881(2)$ & $31(1)$ \\
\hline$C(1 A)$ & $4675(4)$ & $4222(3)$ & $3137(3)$ & $35(2)$ \\
\hline$C(2 A)$ & $5207(12)$ & $4545(6)$ & $3578(12)$ & $37(3)$ \\
\hline$C(3 A)$ & $4083(5)$ & $4580(4)$ & $2959(5)$ & $58(3)$ \\
\hline$C(4 A)$ & $3875(5)$ & $3354(4)$ & $2724(8)$ & $88(5)$ \\
\hline$C(5 A)$ & $4090(9)$ & $3398(7)$ & $2107(8)$ & $139(10)$ \\
\hline$C(6 A)$ & $3655(10)$ & $2762(10)$ & $2817(15)$ & $121(13)$ \\
\hline$C(7 A)$ & $4120(7)$ & $3661(4)$ & $4068(7)$ & $85(4)$ \\
\hline$C(8 A)$ & $4324(13)$ & $4044(7)$ & $4482(8)$ & $163(10)$ \\
\hline$C(9 A)$ & $4026(18)$ & $2999(15)$ & $4190(30)$ & $380(40)$ \\
\hline & & & & \\
\hline & $4 A)$ & & & \\
\hline
\end{tabular}


Table S3. Bond lengths $[\AA]$ and angles $\left[^{\circ}\right]$ for 1 .

\begin{tabular}{|c|c|c|c|}
\hline $\mathrm{Rh}(1)-\mathrm{H}(108)$ & $1.79(4)$ & $\mathrm{Rh}(1)-\mathrm{H}(101)$ & $1.84(4)$ \\
\hline $\mathrm{Rh}(1)-\mathrm{H}(111)$ & $1.85(4)$ & $\operatorname{Rh}(1)-P(1)$ & $2.2359(11)$ \\
\hline $\mathrm{Rh}(1)-\mathrm{Rh}(5)$ & $2.7762(4)$ & $\mathrm{Rh}(1)-\mathrm{Rh}(2)$ & $2.7868(4)$ \\
\hline $\mathrm{Rh}(1)-\mathrm{Rh}(4)$ & $2.8606(4)$ & $\mathrm{Rh}(1)-\mathrm{Rh}(3)$ & $3.0597(5)$ \\
\hline $\mathrm{Rh}(2)-\mathrm{H}(108)$ & $1.79(4)$ & $\mathrm{Rh}(2)-\mathrm{H}(106)$ & $1.81(3)$ \\
\hline $\mathrm{Rh}(2)-\mathrm{H}(105)$ & $1.83(4)$ & $\mathrm{Rh}(2)-\mathrm{H}(107)$ & $1.86(4)$ \\
\hline $\mathrm{Rh}(2)-\mathrm{P}(2)$ & $2.2556(9)$ & $\operatorname{Rh}(2)-\operatorname{Rh}(6)$ & $2.7024(3)$ \\
\hline $\operatorname{Rh}(2)-\operatorname{Rh}(5)$ & $2.7479(4)$ & $\operatorname{Rh}(2)-\operatorname{Rh}(3)$ & $2.8547(4)$ \\
\hline $\mathrm{Rh}(3)-\mathrm{H}(104)$ & $1.80(4)$ & $\mathrm{Rh}(3)-\mathrm{H}(105)$ & $1.84(4)$ \\
\hline $\mathrm{Rh}(3)-\mathrm{H}(109)$ & $1.85(4)$ & $\operatorname{Rh}(3)-P(3)$ & $2.2484(9)$ \\
\hline $\operatorname{Rh}(3)-\operatorname{Rh}(6)$ & $2.7826(3)$ & $\operatorname{Rh}(3)-\operatorname{Rh}(4)$ & $2.7878(4)$ \\
\hline $\mathrm{Rh}(4)-\mathrm{H}(102)$ & $1.77(3)$ & $\mathrm{Rh}(4)-\mathrm{H}(104)$ & $1.80(4)$ \\
\hline $\mathrm{Rh}(4)-\mathrm{H}(101)$ & $1.82(4)$ & $\mathrm{Rh}(4)-\mathrm{H}(103)$ & $1.91(5)$ \\
\hline $\mathrm{Rh}(4)-\mathrm{P}(4)$ & $2.2612(9)$ & $\operatorname{Rh}(4)-\operatorname{Rh}(6)$ & $2.7181(3)$ \\
\hline $\operatorname{Rh}(4)-\operatorname{Rh}(5)$ & $2.7193(3)$ & $\mathrm{Rh}(5)-\mathrm{H}(110)$ & $1.76(3)$ \\
\hline $\mathrm{Rh}(5)-\mathrm{H}(102)$ & $1.78(3)$ & $\mathrm{Rh}(5)-\mathrm{H}(111)$ & $1.84(4)$ \\
\hline $\mathrm{Rh}(5)-\mathrm{H}(107)$ & $1.84(4)$ & $\operatorname{Rh}(5)-P(5)$ & $2.2475(9)$ \\
\hline $\operatorname{Rh}(5)-\operatorname{Rh}(6)$ & $2.7329(3)$ & $\mathrm{Rh}(6)-\mathrm{H}(110)$ & $1.76(3)$ \\
\hline $\mathrm{Rh}(6)-\mathrm{H}(106)$ & $1.83(3)$ & $\mathrm{Rh}(6)-\mathrm{H}(109)$ & $1.83(4)$ \\
\hline $\mathrm{Rh}(6)-\mathrm{H}(103)$ & $1.90(5)$ & $\operatorname{Rh}(6)-P(6)$ & $2.2374(8)$ \\
\hline$P(1)-C(7)$ & $1.719(10)$ & $P(1)-C(4 A)$ & $1.740(10)$ \\
\hline$P(1)-C(4)$ & $1.790(9)$ & $P(1)-C(1 A)$ & $1.889(8)$ \\
\hline$P(1)-C(1)$ & $1.935(9)$ & $P(1)-C(7 A)$ & $2.062(10)$ \\
\hline$P(2)-C(16)$ & $1.853(4)$ & $P(2)-C(13)$ & $1.858(4)$ \\
\hline$P(2)-C(10)$ & $1.860(4)$ & $P(3)-C(19)$ & $1.841(4)$ \\
\hline$P(3)-C(22)$ & $1.858(3)$ & $P(3)-C(25)$ & $1.862(4)$ \\
\hline$P(4)-C(34)$ & $1.849(4)$ & $\mathrm{P}(4)-\mathrm{C}(28)$ & $1.855(4)$ \\
\hline$P(4)-C(31)$ & $1.856(4)$ & $P(5)-C(43)$ & $1.844(4)$ \\
\hline$P(5)-C(37)$ & $1.849(4)$ & $P(5)-C(40)$ & $1.853(4)$ \\
\hline$P(6)-C(52)$ & $1.843(4)$ & $\mathrm{P}(6)-\mathrm{C}(49)$ & $1.852(3)$ \\
\hline$P(6)-C(46)$ & $1.854(3)$ & $C(1)-C(2)$ & $1.553(13)$ \\
\hline$C(1)-C(3)$ & $1.563(12)$ & $C(4)-C(5)$ & $1.49(3)$ \\
\hline$C(4)-C(6)$ & $1.63(2)$ & $\mathrm{C}(05)-\mathrm{B}(91)$ & $1.569(10)$ \\
\hline $\mathrm{C}(05)-\mathrm{B}(90)$ & $1.605(10)$ & $\mathrm{C}(05)-\mathrm{B}(86)$ & $1.693(14)$ \\
\hline $\mathrm{C}(05)-\mathrm{B}(84)$ & $1.796(9)$ & $\mathrm{C}(05)-\mathrm{B}(81)$ & $1.864(10)$ \\
\hline $\mathrm{C}(06)-\mathrm{B}(81)$ & $1.616(11)$ & $\mathrm{C}(06)-\mathrm{B}(82)$ & $1.639(11)$ \\
\hline $\mathrm{C}(06)-\mathrm{B}(85)$ & $1.704(14)$ & $C(06)-B(87)$ & $1.784(11)$ \\
\hline $\mathrm{C}(06)-\mathrm{B}(91)$ & $1.846(11)$ & $C(7)-C(8)$ & $1.44(2)$ \\
\hline$C(7)-C(9)$ & $1.547(13)$ & $C(10)-C(12)$ & $1.523(6)$ \\
\hline $\mathrm{C}(10)-\mathrm{C}(11)$ & $1.535(6)$ & $C(13)-C(14)$ & $1.527(6)$ \\
\hline$C(13)-C(15)$ & $1.540(5)$ & $C(16)-C(17)$ & $1.531(6)$ \\
\hline $\mathrm{C}(16)-\mathrm{C}(18)$ & $1.533(6)$ & $C(19)-C(21)$ & $1.522(6)$ \\
\hline$C(19)-C(20)$ & $1.535(6)$ & $C(22)-C(24)$ & $1.529(6)$ \\
\hline$C(22)-C(23)$ & $1.532(5)$ & $C(25)-C(26)$ & $1.527(6)$ \\
\hline $\mathrm{C}(25)-\mathrm{C}(27)$ & $1.544(6)$ & $C(28)-C(30)$ & $1.521(6)$ \\
\hline $\mathrm{C}(28)-\mathrm{C}(29)$ & $1.534(5)$ & $C(31)-C(33)$ & $1.527(6)$ \\
\hline$C(31)-C(32)$ & $1.538(5)$ & $C(34)-C(36)$ & $1.530(5)$ \\
\hline$C(34)-C(35)$ & $1.534(6)$ & $C(37)-C(39)$ & $1.523(6)$ \\
\hline $\mathrm{C}(37)-\mathrm{C}(38)$ & $1.545(5)$ & $\mathrm{C}(40)-\mathrm{C}(41)$ & $1.517(6)$ \\
\hline$C(40)-C(42)$ & $1.533(6)$ & $C(43)-C(45)$ & $1.532(5)$ \\
\hline $\mathrm{C}(43)-\mathrm{C}(44)$ & $1.532(5)$ & $\mathrm{C}(46)-\mathrm{C}(48)$ & $1.522(5)$ \\
\hline $\mathrm{C}(46)-\mathrm{C}(47)$ & $1.533(5)$ & $\mathrm{C}(49)-\mathrm{C}(51)$ & $1.520(5)$ \\
\hline
\end{tabular}




\begin{tabular}{|c|c|c|c|}
\hline$C(49)-C(50)$ & $1.542(5)$ & $C(52)-C(53)$ & $1.528(5)$ \\
\hline$C(52)-C(54)$ & $1.530(5)$ & $\mathrm{C}(61)-\mathrm{B}(64)$ & $1.698(5)$ \\
\hline$C(61)-B(65)$ & $1.703(6)$ & $C(61)-B(63)$ & $1.712(5)$ \\
\hline$C(61)-B(62)$ & $1.713(6)$ & $\mathrm{C}(61)-\mathrm{B}(66)$ & $1.715(5)$ \\
\hline$C(62)-B(62)$ & $1.590(6)$ & $\mathrm{C}(63)-\mathrm{B}(63)$ & $1.590(5)$ \\
\hline$C(64)-B(64)$ & $1.592(5)$ & $C(65)-B(65)$ & $1.592(6)$ \\
\hline$C(66)-B(66)$ & $1.598(6)$ & $\mathrm{C}(67)-\mathrm{B}(67)$ & $1.605(6)$ \\
\hline $\mathrm{C}(68)-\mathrm{B}(68)$ & $1.596(5)$ & $\mathrm{C}(69)-\mathrm{B}(69)$ & $1.607(5)$ \\
\hline$C(70)-B(70)$ & $1.603(5)$ & $\mathrm{C}(71)-\mathrm{B}(72)$ & $1.601(5)$ \\
\hline$C(81)-B(81)$ & $1.595(6)$ & $\mathrm{C}(82)-\mathrm{B}(82)$ & $1.596(5)$ \\
\hline$C(83)-B(83)$ & $1.603(6)$ & $\mathrm{C}(84)-\mathrm{B}(84)$ & $1.599(6)$ \\
\hline $\mathrm{C}(85)-\mathrm{B}(85)$ & $1.625(14)$ & $\mathrm{C}(86)-\mathrm{B}(86)$ & $1.614(13)$ \\
\hline $\mathrm{C}(87)-\mathrm{B}(87)$ & $1.595(5)$ & $\mathrm{C}(88)-\mathrm{B}(88)$ & $1.607(6)$ \\
\hline $\mathrm{C}(89)-\mathrm{B}(89)$ & $1.605(5)$ & $\mathrm{C}(90)-\mathrm{B}(90)$ & $1.604(6)$ \\
\hline $\mathrm{C}(91)-\mathrm{B}(91)$ & $1.598(6)$ & $\mathrm{C}(92)-\mathrm{B}(92)$ & $1.594(6)$ \\
\hline $\mathrm{C}(107)-\mathrm{B}(71)$ & $1.600(5)$ & $\mathrm{C}(901)-\mathrm{C}(902)$ & 1.44(3) \\
\hline $\mathrm{C}(901)-\mathrm{C}(903)$ & $1.97(3)$ & $\mathrm{C}(902)-\mathrm{C}(903)$ & 1.358(19) \\
\hline$C(903)-C(904)$ & $1.297(18)$ & $\mathrm{C}(903)-\mathrm{C}(905)$ & 1.52(2) \\
\hline$C(904)-C(905)$ & $0.802(16)$ & $\mathrm{C}(906)-\mathrm{C}(907)$ & $0.793(19)$ \\
\hline C(907)-C(909)\#1 & $2.01(3)$ & $\mathrm{C}(908)-\mathrm{C}(909)$ & $1.10(2)$ \\
\hline C(909)-C(907)\#1 & $2.01(3)$ & $\mathrm{B}(62)-\mathrm{B}(70)$ & $1.767(6)$ \\
\hline $\mathrm{B}(62)-\mathrm{B}(71)$ & $1.771(6)$ & $\mathrm{B}(62)-\mathrm{B}(66)$ & $1.788(7)$ \\
\hline$B(62)-B(63)$ & $1.790(6)$ & $\mathrm{B}(63)-\mathrm{B}(69)$ & $1.768(6)$ \\
\hline $\mathrm{B}(63)-\mathrm{B}(70)$ & $1.771(6)$ & $\mathrm{B}(63)-\mathrm{B}(64)$ & $1.777(6)$ \\
\hline$B(64)-B(69)$ & $1.773(5)$ & $\mathrm{B}(64)-\mathrm{B}(65)$ & $1.777(6)$ \\
\hline$B(64)-B(68)$ & $1.778(6)$ & $\mathrm{B}(65)-\mathrm{B}(68)$ & $1.774(6)$ \\
\hline$B(65)-B(67)$ & $1.775(6)$ & $\mathrm{B}(65)-\mathrm{B}(66)$ & $1.788(6)$ \\
\hline$B(66)-B(67)$ & $1.778(6)$ & $\mathrm{B}(66)-\mathrm{B}(71)$ & $1.780(6)$ \\
\hline$B(67)-B(72)$ & 1.793(6) & $\mathrm{B}(67)-\mathrm{B}(71)$ & $1.794(6)$ \\
\hline$B(67)-B(68)$ & $1.797(6)$ & $\mathrm{B}(68)-\mathrm{B}(69)$ & $1.789(5)$ \\
\hline$B(68)-B(72)$ & $1.794(5)$ & $\mathrm{B}(69)-\mathrm{B}(72)$ & $1.785(5)$ \\
\hline$B(69)-B(70)$ & $1.796(5)$ & $\mathrm{B}(70)-\mathrm{B}(71)$ & $1.792(6)$ \\
\hline$B(70)-B(72)$ & $1.796(5)$ & $\mathrm{B}(71)-\mathrm{B}(72)$ & $1.790(6)$ \\
\hline$B(81)-B(85)$ & $1.600(12)$ & $\mathrm{B}(81)-\mathrm{B}(82)$ & $1.768(6)$ \\
\hline$B(81)-B(83)$ & $1.772(6)$ & $\mathrm{B}(81)-\mathrm{B}(84)$ & $1.785(6)$ \\
\hline$B(81)-B(86)$ & $1.884(13)$ & $\mathrm{B}(82)-\mathrm{B}(88)$ & $1.777(6)$ \\
\hline $\mathrm{B}(82)-\mathrm{B}(83)$ & $1.783(6)$ & $\mathrm{B}(82)-\mathrm{B}(87)$ & $1.791(5)$ \\
\hline$B(82)-B(86)$ & $1.860(13)$ & $\mathrm{B}(83)-\mathrm{B}(84)$ & $1.770(6)$ \\
\hline$B(83)-B(89)$ & $1.780(6)$ & $\mathrm{B}(83)-\mathrm{B}(88)$ & $1.781(6)$ \\
\hline$B(84)-B(85)$ & $1.685(11)$ & $\mathrm{B}(84)-\mathrm{B}(89)$ & $1.771(6)$ \\
\hline$B(84)-B(90)$ & $1.785(7)$ & $B(85)-B(90)$ & $1.875(12)$ \\
\hline$B(85)-B(91)$ & $1.923(12)$ & $\mathrm{B}(86)-\mathrm{B}(91)$ & $1.651(13)$ \\
\hline $\mathrm{B}(86)-\mathrm{B}(87)$ & $1.717(12)$ & $\mathrm{B}(87)-\mathrm{B}(91)$ & $1.773(6)$ \\
\hline$B(87)-B(88)$ & $1.779(6)$ & $B(87)-B(92)$ & $1.783(6)$ \\
\hline$B(88)-B(89)$ & $1.779(6)$ & $B(88)-B(92)$ & $1.784(6)$ \\
\hline$B(89)-B(90)$ & $1.769(6)$ & $\mathrm{B}(89)-\mathrm{B}(92)$ & $1.786(6)$ \\
\hline$B(90)-B(92)$ & $1.778(6)$ & $\mathrm{B}(90)-\mathrm{B}(91)$ & $1.790(6)$ \\
\hline$B(91)-B(92)$ & $1.774(6)$ & $C(1 A)-C(3 A)$ & $1.540(11)$ \\
\hline$C(1 A)-C(2 A)$ & $1.55(3)$ & $C(4 A)-C(5 A)$ & $1.53(2)$ \\
\hline$C(4 A)-C(6 A)$ & $1.61(3)$ & $C(7 A)-C(8 A)$ & $1.33(2)$ \\
\hline$C(7 A)-C(9 A)$ & $1.73(4)$ & & \\
\hline $\mathrm{H}(108)-\mathrm{Rh}(1)-\mathrm{H}(101)$ & 162(3) & $\mathrm{H}(108)-\mathrm{Rh}(1)-\mathrm{H}(111)$ & 91(3) \\
\hline $\mathrm{H}(101)-\mathrm{Rh}(1)-\mathrm{H}(111)$ & $92(3)$ & $\mathrm{H}(108)-\mathrm{Rh}(1)-\mathrm{P}(1)$ & $88.3(17)$ \\
\hline
\end{tabular}




\begin{tabular}{|c|c|c|c|}
\hline $\mathrm{H}(101)-\mathrm{Rh}(1)-\mathrm{P}(1)$ & $109.4(17)$ & $\mathrm{H}(111)-\mathrm{Rh}(1)-\mathrm{P}(1)$ & $92.0(13)$ \\
\hline $\mathrm{H}(108)-\operatorname{Rh}(1)-\operatorname{Rh}(5)$ & $85(2)$ & $\mathrm{H}(101)-\mathrm{Rh}(1)-\mathrm{Rh}(5)$ & $86.5(18)$ \\
\hline $\mathrm{H}(111)-\operatorname{Rh}(1)-\operatorname{Rh}(5)$ & $40.9(13)$ & $\mathrm{P}(1)-\operatorname{Rh}(1)-\operatorname{Rh}(5)$ & $131.88(4)$ \\
\hline $\mathrm{H}(108)-\mathrm{Rh}(1)-\mathrm{Rh}(2)$ & $39.0(17)$ & $\mathrm{H}(101)-\mathrm{Rh}(1)-\mathrm{Rh}(2)$ & $123.2(17)$ \\
\hline $\mathrm{H}(111)-\operatorname{Rh}(1)-\operatorname{Rh}(2)$ & $88.5(14)$ & $\mathrm{P}(1)-\mathrm{Rh}(1)-\mathrm{Rh}(2)$ & $127.27(3)$ \\
\hline $\operatorname{Rh}(5)-\operatorname{Rh}(1)-\operatorname{Rh}(2)$ & $59.202(9)$ & $\mathrm{H}(108)-\mathrm{Rh}(1)-\mathrm{Rh}(4)$ & $124.3(17)$ \\
\hline $\mathrm{H}(101)-\mathrm{Rh}(1)-\mathrm{Rh}(4)$ & $38.5(17)$ & $\mathrm{H}(111)-\mathrm{Rh}(1)-\mathrm{Rh}(4)$ & $84.9(15)$ \\
\hline$P(1)-R h(1)-R h(4)$ & $147.26(3)$ & $\operatorname{Rh}(5)-\operatorname{Rh}(1)-\operatorname{Rh}(4)$ & $57.663(9)$ \\
\hline $\operatorname{Rh}(2)-\operatorname{Rh}(1)-\operatorname{Rh}(4)$ & $85.297(11)$ & $\mathrm{H}(108)-\mathrm{Rh}(1)-\operatorname{Rh}(3)$ & $85.6(19)$ \\
\hline $\mathrm{H}(101)-\mathrm{Rh}(1)-\mathrm{Rh}(3)$ & $78(2)$ & $\mathrm{H}(111)-\mathrm{Rh}(1)-\mathrm{Rh}(3)$ & $127.8(13)$ \\
\hline$P(1)-R h(1)-R h(3)$ & $139.75(4)$ & $\mathrm{Rh}(5)-\operatorname{Rh}(1)-\operatorname{Rh}(3)$ & $87.067(11)$ \\
\hline $\operatorname{Rh}(2)-\operatorname{Rh}(1)-\operatorname{Rh}(3)$ & $58.231(10)$ & $\operatorname{Rh}(4)-\operatorname{Rh}(1)-\operatorname{Rh}(3)$ & $56.061(9)$ \\
\hline $\mathrm{H}(108)-\mathrm{Rh}(2)-\mathrm{H}(106)$ & $174(2)$ & $\mathrm{H}(108)-\mathrm{Rh}(2)-\mathrm{H}(105)$ & $92(3)$ \\
\hline $\mathrm{H}(106)-\mathrm{Rh}(2)-\mathrm{H}(105)$ & $90(2)$ & $\mathrm{H}(108)-\mathrm{Rh}(2)-\mathrm{H}(107)$ & $84(3)$ \\
\hline $\mathrm{H}(106)-\mathrm{Rh}(2)-\mathrm{H}(107)$ & $94(2)$ & $\mathrm{H}(105)-\mathrm{Rh}(2)-\mathrm{H}(107)$ & $172(2)$ \\
\hline $\mathrm{H}(108)-\mathrm{Rh}(2)-\mathrm{P}(2)$ & $93.9(17)$ & $\mathrm{H}(106)-\mathrm{Rh}(2)-\mathrm{P}(2)$ & $91.7(12)$ \\
\hline $\mathrm{H}(105)-\mathrm{Rh}(2)-\mathrm{P}(2)$ & $84.8(14)$ & $\mathrm{H}(107)-\mathrm{Rh}(2)-\mathrm{P}(2)$ & $102.5(13)$ \\
\hline $\mathrm{H}(108)-\mathrm{Rh}(2)-\mathrm{Rh}(6)$ & $132.2(17)$ & $\mathrm{H}(106)-\mathrm{Rh}(2)-\mathrm{Rh}(6)$ & $42.3(12)$ \\
\hline $\mathrm{H}(105)-\mathrm{Rh}(2)-\mathrm{Rh}(6)$ & $88.3(16)$ & $\mathrm{H}(107)-\mathrm{Rh}(2)-\operatorname{Rh}(6)$ & $89.5(15)$ \\
\hline$P(2)-R h(2)-R h(6)$ & 133.56(3) & $\mathrm{H}(108)-\operatorname{Rh}(2)-\operatorname{Rh}(5)$ & $85.4(19)$ \\
\hline $\mathrm{H}(106)-\mathrm{Rh}(2)-\mathrm{Rh}(5)$ & $89.6(13)$ & $\mathrm{H}(105)-\operatorname{Rh}(2)-\operatorname{Rh}(5)$ & $130.9(14)$ \\
\hline $\mathrm{H}(107)-\operatorname{Rh}(2)-\operatorname{Rh}(5)$ & $41.9(13)$ & $\mathrm{P}(2)-\operatorname{Rh}(2)-\operatorname{Rh}(5)$ & $144.33(3)$ \\
\hline $\operatorname{Rh}(6)-\operatorname{Rh}(2)-\operatorname{Rh}(5)$ & $60.181(9)$ & $\mathrm{H}(108)-\mathrm{Rh}(2)-\mathrm{Rh}(1)$ & $38.9(17)$ \\
\hline $\mathrm{H}(106)-\mathrm{Rh}(2)-\mathrm{Rh}(1)$ & $135.7(12)$ & $\mathrm{H}(105)-\operatorname{Rh}(2)-\operatorname{Rh}(1)$ & $88.0(17)$ \\
\hline $\mathrm{H}(107)-\mathrm{Rh}(2)-\mathrm{Rh}(1)$ & $84.0(15)$ & $\mathrm{P}(2)-\mathrm{Rh}(2)-\mathrm{Rh}(1)$ & $132.04(3)$ \\
\hline $\operatorname{Rh}(6)-\operatorname{Rh}(2)-\operatorname{Rh}(1)$ & $93.377(11)$ & $\operatorname{Rh}(5)-\operatorname{Rh}(2)-\operatorname{Rh}(1)$ & $60.207(10)$ \\
\hline $\mathrm{H}(108)-\mathrm{Rh}(2)-\mathrm{Rh}(3)$ & $92(2)$ & $\mathrm{H}(106)-\operatorname{Rh}(2)-\operatorname{Rh}(3)$ & $85.5(14)$ \\
\hline $\mathrm{H}(105)-\operatorname{Rh}(2)-\operatorname{Rh}(3)$ & $39.2(14)$ & $\mathrm{H}(107)-\operatorname{Rh}(2)-\operatorname{Rh}(3)$ & $133.7(13)$ \\
\hline$P(2)-\operatorname{Rh}(2)-\operatorname{Rh}(3)$ & 123.81(3) & $\operatorname{Rh}(6)-\operatorname{Rh}(2)-\operatorname{Rh}(3)$ & $60.021(9)$ \\
\hline $\operatorname{Rh}(5)-\operatorname{Rh}(2)-\operatorname{Rh}(3)$ & $91.825(11)$ & $\operatorname{Rh}(1)-\operatorname{Rh}(2)-\operatorname{Rh}(3)$ & $65.676(11)$ \\
\hline $\mathrm{H}(104)-\mathrm{Rh}(3)-\mathrm{H}(105)$ & $163(2)$ & $\mathrm{H}(104)-\mathrm{Rh}(3)-\mathrm{H}(109)$ & $89(3)$ \\
\hline $\mathrm{H}(105)-\mathrm{Rh}(3)-\mathrm{H}(109)$ & $94(3)$ & $\mathrm{H}(104)-\mathrm{Rh}(3)-\mathrm{P}(3)$ & $88.3(13)$ \\
\hline $\mathrm{H}(105)-\mathrm{Rh}(3)-\mathrm{P}(3)$ & $108.2(14)$ & $\mathrm{H}(109)-\mathrm{Rh}(3)-\mathrm{P}(3)$ & $96.4(15)$ \\
\hline $\mathrm{H}(104)-\operatorname{Rh}(3)-\operatorname{Rh}(6)$ & $85.3(15)$ & $\mathrm{H}(105)-\mathrm{Rh}(3)-\mathrm{Rh}(6)$ & $85.7(16)$ \\
\hline $\mathrm{H}(109)-\operatorname{Rh}(3)-\operatorname{Rh}(6)$ & 40.6(15) & $\mathrm{P}(3)-\operatorname{Rh}(3)-\operatorname{Rh}(6)$ & $136.60(3)$ \\
\hline $\mathrm{H}(104)-\operatorname{Rh}(3)-\operatorname{Rh}(4)$ & $39.2(13)$ & $\mathrm{H}(105)-\mathrm{Rh}(3)-\mathrm{Rh}(4)$ & $124.1(14)$ \\
\hline $\mathrm{H}(109)-\mathrm{Rh}(3)-\mathrm{Rh}(4)$ & $85.1(17)$ & $\mathrm{P}(3)-\operatorname{Rh}(3)-\operatorname{Rh}(4)$ & $127.50(2)$ \\
\hline $\operatorname{Rh}(6)-\operatorname{Rh}(3)-\operatorname{Rh}(4)$ & $58.412(9)$ & $\mathrm{H}(104)-\operatorname{Rh}(3)-\operatorname{Rh}(2)$ & $124.6(13)$ \\
\hline $\mathrm{H}(105)-\mathrm{Rh}(3)-\mathrm{Rh}(2)$ & $39.0(14)$ & $\mathrm{H}(109)-\mathrm{Rh}(3)-\mathrm{Rh}(2)$ & $86.4(16)$ \\
\hline$P(3)-R h(3)-R h(2)$ & $147.09(3)$ & $\operatorname{Rh}(6)-\operatorname{Rh}(3)-\operatorname{Rh}(2)$ & $57.273(9)$ \\
\hline $\operatorname{Rh}(4)-\operatorname{Rh}(3)-\operatorname{Rh}(2)$ & $85.392(10)$ & $\mathrm{H}(104)-\mathrm{Rh}(3)-\mathrm{Rh}(1)$ & $84.9(15)$ \\
\hline $\mathrm{H}(105)-\mathrm{Rh}(3)-\mathrm{Rh}(1)$ & $79.9(17)$ & $H(109)-\operatorname{Rh}(3)-R h(1)$ & 126.8(15) \\
\hline$P(3)-R h(3)-R h(1)$ & $135.97(3)$ & $\operatorname{Rh}(6)-\operatorname{Rh}(3)-\operatorname{Rh}(1)$ & $86.134(10)$ \\
\hline $\operatorname{Rh}(4)-\operatorname{Rh}(3)-\operatorname{Rh}(1)$ & $58.354(9)$ & $\operatorname{Rh}(2)-\operatorname{Rh}(3)-\operatorname{Rh}(1)$ & $56.094(9)$ \\
\hline $\mathrm{H}(102)-\mathrm{Rh}(4)-\mathrm{H}(104)$ & $172.9(19)$ & $\mathrm{H}(102)-\mathrm{Rh}(4)-\mathrm{H}(101)$ & $93(3)$ \\
\hline $\mathrm{H}(104)-\mathrm{Rh}(4)-\mathrm{H}(101)$ & $88(3)$ & $\mathrm{H}(102)-\mathrm{Rh}(4)-\mathrm{H}(103)$ & $88(3)$ \\
\hline $\mathrm{H}(104)-\mathrm{Rh}(4)-\mathrm{H}(103)$ & $90(3)$ & $\mathrm{H}(101)-\mathrm{Rh}(4)-\mathrm{H}(103)$ & $174(2)$ \\
\hline $\mathrm{H}(102)-\mathrm{Rh}(4)-\mathrm{P}(4)$ & $95.0(12)$ & $\mathrm{H}(104)-\mathrm{Rh}(4)-\mathrm{P}(4)$ & $91.9(14)$ \\
\hline $\mathrm{H}(101)-\mathrm{Rh}(4)-\mathrm{P}(4)$ & $91.5(17)$ & $\mathrm{H}(103)-\mathrm{Rh}(4)-\mathrm{P}(4)$ & $94.2(15)$ \\
\hline $\mathrm{H}(102)-\mathrm{Rh}(4)-\mathrm{Rh}(6)$ & $86.5(13)$ & $\mathrm{H}(104)-\operatorname{Rh}(4)-\operatorname{Rh}(6)$ & $87.3(15)$ \\
\hline $\mathrm{H}(101)-\operatorname{Rh}(4)-\operatorname{Rh}(6)$ & $130.0(17)$ & $\mathrm{H}(103)-\mathrm{Rh}(4)-\operatorname{Rh}(6)$ & $44.3(15)$ \\
\hline $\mathrm{P}(4)-\mathrm{Rh}(4)-\mathrm{Rh}(6)$ & $138.42(2)$ & $\mathrm{H}(102)-\mathrm{Rh}(4)-\mathrm{Rh}(5)$ & $40.0(12)$ \\
\hline $\mathrm{H}(104)-\mathrm{Rh}(4)-\mathrm{Rh}(5)$ & 133.2(14) & $\mathrm{H}(101)-\operatorname{Rh}(4)-\operatorname{Rh}(5)$ & $88.6(18)$ \\
\hline $\mathrm{H}(103)-\mathrm{Rh}(4)-\mathrm{Rh}(5)$ & $88.3(18)$ & $\mathrm{P}(4)-\mathrm{Rh}(4)-\mathrm{Rh}(5)$ & $134.88(3)$ \\
\hline
\end{tabular}




\begin{tabular}{|c|c|c|c|}
\hline $\mathrm{Rh}(6)-\mathrm{Rh}(4)-\mathrm{Rh}(5)$ & $60.347(9)$ & $\mathrm{H}(102)-\mathrm{Rh}(4)-\mathrm{Rh}(3)$ & 133.9(12) \\
\hline $\mathrm{H}(104)-\mathrm{Rh}(4)-\mathrm{Rh}(3)$ & $39.3(14)$ & $\mathrm{H}(101)-\operatorname{Rh}(4)-\operatorname{Rh}(3)$ & $86(2)$ \\
\hline $\mathrm{H}(103)-\mathrm{Rh}(4)-\mathrm{Rh}(3)$ & 89.1(19) & $\mathrm{P}(4)-\operatorname{Rh}(4)-\operatorname{Rh}(3)$ & 131.12(3) \\
\hline $\mathrm{Rh}(6)-\mathrm{Rh}(4)-\mathrm{Rh}(3)$ & $60.699(9)$ & $\mathrm{Rh}(5)-\mathrm{Rh}(4)-\mathrm{Rh}(3)$ & $93.911(10)$ \\
\hline $\mathrm{H}(102)-\mathrm{Rh}(4)-\mathrm{Rh}(1)$ & $85.4(13)$ & $\mathrm{H}(104)-\operatorname{Rh}(4)-\operatorname{Rh}(1)$ & 91.2(15) \\
\hline $\mathrm{H}(101)-\mathrm{Rh}(4)-\mathrm{Rh}(1)$ & $38.9(17)$ & $\mathrm{H}(103)-\operatorname{Rh}(4)-\operatorname{Rh}(1)$ & $135.5(15)$ \\
\hline $\mathrm{P}(4)-\mathrm{Rh}(4)-\mathrm{Rh}(1)$ & $130.15(2)$ & $\mathrm{Rh}(6)-\mathrm{Rh}(4)-\mathrm{Rh}(1)$ & $91.422(10)$ \\
\hline $\operatorname{Rh}(5)-\operatorname{Rh}(4)-\operatorname{Rh}(1)$ & $59.611(10)$ & $\operatorname{Rh}(3)-\operatorname{Rh}(4)-\operatorname{Rh}(1)$ & $65.585(11)$ \\
\hline $\mathrm{H}(110)-\mathrm{Rh}(5)-\mathrm{H}(102)$ & $89(2)$ & $\mathrm{H}(110)-\mathrm{Rh}(5)-\mathrm{H}(111)$ & $173.4(18)$ \\
\hline $\mathrm{H}(102)-\mathrm{Rh}(5)-\mathrm{H}(111)$ & $90(2)$ & $\mathrm{H}(110)-\mathrm{Rh}(5)-\mathrm{H}(107)$ & 92(2) \\
\hline $\mathrm{H}(102)-\mathrm{Rh}(5)-\mathrm{H}(107)$ & $170.8(17)$ & $\mathrm{H}(111)-\mathrm{Rh}(5)-\mathrm{H}(107)$ & $88(2)$ \\
\hline$H(110)-R h(5)-P(5)$ & $95.1(13)$ & $\mathrm{H}(102)-\mathrm{Rh}(5)-\mathrm{P}(5)$ & $95.1(12)$ \\
\hline $\mathrm{H}(111)-\mathrm{Rh}(5)-\mathrm{P}(5)$ & $91.5(13)$ & $\mathrm{H}(107)-\mathrm{Rh}(5)-\mathrm{P}(5)$ & $94.0(13)$ \\
\hline $\mathrm{H}(110)-\mathrm{Rh}(5)-\mathrm{Rh}(4)$ & $85.9(15)$ & $\mathrm{H}(102)-\operatorname{Rh}(5)-\operatorname{Rh}(4)$ & $39.7(12)$ \\
\hline $\mathrm{H}(111)-\mathrm{Rh}(5)-\mathrm{Rh}(4)$ & $89.4(15)$ & $\mathrm{H}(107)-\operatorname{Rh}(5)-\operatorname{Rh}(4)$ & $131.2(13)$ \\
\hline$P(5)-R h(5)-R h(4)$ & 134.76(3) & $\mathrm{H}(110)-\operatorname{Rh}(5)-\operatorname{Rh}(6)$ & $39.2(13)$ \\
\hline $\mathrm{H}(102)-\mathrm{Rh}(5)-\mathrm{Rh}(6)$ & $85.8(13)$ & $\mathrm{H}(111)-\operatorname{Rh}(5)-\operatorname{Rh}(6)$ & 134.2(13) \\
\hline $\mathrm{H}(107)-\mathrm{Rh}(5)-\mathrm{Rh}(6)$ & $89.0(15)$ & $P(5)-\operatorname{Rh}(5)-\operatorname{Rh}(6)$ & 134.31(3) \\
\hline $\operatorname{Rh}(4)-\operatorname{Rh}(5)-\operatorname{Rh}(6)$ & $59.804(9)$ & $\mathrm{H}(110)-\operatorname{Rh}(5)-\operatorname{Rh}(2)$ & $85.3(15)$ \\
\hline $\mathrm{H}(102)-\mathrm{Rh}(5)-\mathrm{Rh}(2)$ & $128.5(12)$ & $\mathrm{H}(111)-\operatorname{Rh}(5)-\operatorname{Rh}(2)$ & $90.0(14)$ \\
\hline $\mathrm{H}(107)-\mathrm{Rh}(5)-\mathrm{Rh}(2)$ & $42.5(13)$ & $P(5)-R h(5)-R h(2)$ & 136.38(3) \\
\hline $\operatorname{Rh}(4)-\operatorname{Rh}(5)-\operatorname{Rh}(2)$ & $88.837(10)$ & $\operatorname{Rh}(6)-\operatorname{Rh}(5)-\operatorname{Rh}(2)$ & $59.084(9)$ \\
\hline $\mathrm{H}(110)-\mathrm{Rh}(5)-\mathrm{Rh}(1)$ & 132.1(13) & $\mathrm{H}(102)-\operatorname{Rh}(5)-\operatorname{Rh}(1)$ & $87.9(13)$ \\
\hline $\mathrm{H}(111)-\mathrm{Rh}(5)-\mathrm{Rh}(1)$ & $41.2(13)$ & $\mathrm{H}(107)-\operatorname{Rh}(5)-\operatorname{Rh}(1)$ & $84.7(15)$ \\
\hline$P(5)-R h(5)-R h(1)$ & $132.74(3)$ & $\operatorname{Rh}(4)-\operatorname{Rh}(5)-\operatorname{Rh}(1)$ & $62.726(10)$ \\
\hline $\operatorname{Rh}(6)-\operatorname{Rh}(5)-\operatorname{Rh}(1)$ & $92.949(12)$ & $\operatorname{Rh}(2)-\operatorname{Rh}(5)-\operatorname{Rh}(1)$ & $60.591(10)$ \\
\hline $\mathrm{H}(110)-\mathrm{Rh}(6)-\mathrm{H}(106)$ & $92(2)$ & $\mathrm{H}(110)-\mathrm{Rh}(6)-\mathrm{H}(109)$ & $173(2)$ \\
\hline $\mathrm{H}(106)-\mathrm{Rh}(6)-\mathrm{H}(109)$ & 91(2) & $\mathrm{H}(110)-\mathrm{Rh}(6)-\mathrm{H}(103)$ & $88(3)$ \\
\hline $\mathrm{H}(106)-\mathrm{Rh}(6)-\mathrm{H}(103)$ & $176(2)$ & $\mathrm{H}(109)-\mathrm{Rh}(6)-\mathrm{H}(103)$ & 89(3) \\
\hline$H(110)-R h(6)-P(6)$ & $93.9(13)$ & $\mathrm{H}(106)-\mathrm{Rh}(6)-\mathrm{P}(6)$ & $93.8(11)$ \\
\hline $\mathrm{H}(109)-\mathrm{Rh}(6)-\mathrm{P}(6)$ & 92.2(15) & $\mathrm{H}(103)-\mathrm{Rh}(6)-\mathrm{P}(6)$ & $90.0(15)$ \\
\hline $\mathrm{H}(110)-\mathrm{Rh}(6)-\mathrm{Rh}(2)$ & $86.7(15)$ & $\mathrm{H}(106)-\operatorname{Rh}(6)-\operatorname{Rh}(2)$ & $41.8(11)$ \\
\hline $\mathrm{H}(109)-\mathrm{Rh}(6)-\mathrm{Rh}(2)$ & 91.4(17) & $\mathrm{H}(103)-\mathrm{Rh}(6)-\mathrm{Rh}(2)$ & 134.4(15) \\
\hline$P(6)-R h(6)-R h(2)$ & $135.49(2)$ & $\mathrm{H}(110)-\mathrm{Rh}(6)-\mathrm{Rh}(4)$ & $85.9(15)$ \\
\hline $\mathrm{H}(106)-\mathrm{Rh}(6)-\mathrm{Rh}(4)$ & 131.5(11) & $\mathrm{H}(109)-\mathrm{Rh}(6)-\mathrm{Rh}(4)$ & $87.4(17)$ \\
\hline $\mathrm{H}(103)-\mathrm{Rh}(6)-\mathrm{Rh}(4)$ & $44.7(15)$ & $\mathrm{P}(6)-\mathrm{Rh}(6)-\mathrm{Rh}(4)$ & $134.67(2)$ \\
\hline $\operatorname{Rh}(2)-\operatorname{Rh}(6)-\operatorname{Rh}(4)$ & $89.810(10)$ & $\mathrm{H}(110)-\operatorname{Rh}(6)-\operatorname{Rh}(5)$ & $39.2(13)$ \\
\hline $\mathrm{H}(106)-\mathrm{Rh}(6)-\mathrm{Rh}(5)$ & $89.7(13)$ & $\mathrm{H}(109)-\operatorname{Rh}(6)-\operatorname{Rh}(5)$ & 134.6(15) \\
\hline $\mathrm{H}(103)-\mathrm{Rh}(6)-\mathrm{Rh}(5)$ & $88.2(19)$ & $\mathrm{P}(6)-\mathrm{Rh}(6)-\mathrm{Rh}(5)$ & $133.06(2)$ \\
\hline $\operatorname{Rh}(2)-\operatorname{Rh}(6)-\operatorname{Rh}(5)$ & $60.735(10)$ & $\operatorname{Rh}(4)-\operatorname{Rh}(6)-\operatorname{Rh}(5)$ & $59.849(9)$ \\
\hline $\mathrm{H}(110)-\mathrm{Rh}(6)-\mathrm{Rh}(3)$ & $132.9(13)$ & $\mathrm{H}(106)-\operatorname{Rh}(6)-\operatorname{Rh}(3)$ & $87.3(14)$ \\
\hline $\mathrm{H}(109)-\mathrm{Rh}(6)-\mathrm{Rh}(3)$ & $41.0(15)$ & $\mathrm{H}(103)-\operatorname{Rh}(6)-\operatorname{Rh}(3)$ & $89.6(19)$ \\
\hline$P(6)-\operatorname{Rh}(6)-\operatorname{Rh}(3)$ & $133.17(2)$ & $\operatorname{Rh}(2)-\operatorname{Rh}(6)-\operatorname{Rh}(3)$ & $62.706(9)$ \\
\hline $\operatorname{Rh}(4)-\operatorname{Rh}(6)-\operatorname{Rh}(3)$ & $60.889(9)$ & $\operatorname{Rh}(5)-\operatorname{Rh}(6)-\operatorname{Rh}(3)$ & $93.726(11)$ \\
\hline$C(7)-P(1)-C(4 A)$ & $130.5(5)$ & $C(7)-P(1)-C(4)$ & $110.3(6)$ \\
\hline$C(4 A)-P(1)-C(4)$ & $59.9(7)$ & $C(7)-P(1)-C(1 A)$ & 46.4(4) \\
\hline$C(4 A)-P(1)-C(1 A)$ & 101.9(5) & $C(4)-P(1)-C(1 A)$ & $133.4(4)$ \\
\hline$C(7)-P(1)-C(1)$ & $108.4(4)$ & $C(4 A)-P(1)-C(1)$ & $47.8(6)$ \\
\hline$C(4)-P(1)-C(1)$ & $107.0(5)$ & $C(1 A)-P(1)-C(1)$ & $63.3(4)$ \\
\hline$C(7)-P(1)-C(7 A)$ & $57.8(5)$ & $C(4 A)-P(1)-C(7 A)$ & 102.1(7) \\
\hline$C(4)-P(1)-C(7 A)$ & $53.5(6)$ & $C(1 A)-P(1)-C(7 A)$ & $96.6(4)$ \\
\hline$C(1)-P(1)-C(7 A)$ & $131.0(5)$ & $\mathrm{C}(7)-\mathrm{P}(1)-\mathrm{Rh}(1)$ & 108.0(3) \\
\hline$C(4 A)-P(1)-R h(1)$ & 121.1(4) & $\mathrm{C}(4)-\mathrm{P}(1)-\mathrm{Rh}(1)$ & 110.2(3) \\
\hline$C(1 A)-P(1)-R h(1)$ & $115.5(2)$ & $\mathrm{C}(1)-\mathrm{P}(1)-\mathrm{Rh}(1)$ & 113.0(3) \\
\hline
\end{tabular}




\begin{tabular}{|c|c|c|c|}
\hline$C(7 A)-P(1)-R h(1)$ & $116.0(4)$ & $\mathrm{C}(16)-\mathrm{P}(2)-\mathrm{C}(13)$ & $105.96(17)$ \\
\hline$C(16)-P(2)-C(10)$ & 105.32(19) & $\mathrm{C}(13)-\mathrm{P}(2)-\mathrm{C}(10)$ & $105.47(18)$ \\
\hline$C(16)-P(2)-R h(2)$ & $112.81(12)$ & $\mathrm{C}(13)-\mathrm{P}(2)-\mathrm{Rh}(2)$ & 115.92(13) \\
\hline$C(10)-P(2)-R h(2)$ & $110.56(13)$ & $\mathrm{C}(19)-\mathrm{P}(3)-\mathrm{C}(22)$ & $106.47(16)$ \\
\hline$C(19)-P(3)-C(25)$ & $104.37(18)$ & $C(22)-P(3)-C(25)$ & 105.78(18) \\
\hline$C(19)-P(3)-R h(3)$ & $114.72(14)$ & $\mathrm{C}(22)-\mathrm{P}(3)-\mathrm{Rh}(3)$ & $114.60(12)$ \\
\hline$C(25)-P(3)-\operatorname{Rh}(3)$ & $110.03(12)$ & $\mathrm{C}(34)-\mathrm{P}(4)-\mathrm{C}(28)$ & 104.44(17) \\
\hline$C(34)-P(4)-C(31)$ & $103.29(17)$ & $C(28)-P(4)-C(31)$ & $111.05(17)$ \\
\hline $\mathrm{C}(34)-\mathrm{P}(4)-\mathrm{Rh}(4)$ & $111.40(12)$ & $\mathrm{C}(28)-\mathrm{P}(4)-\mathrm{Rh}(4)$ & 113.33(12) \\
\hline $\mathrm{C}(31)-\mathrm{P}(4)-\mathrm{Rh}(4)$ & $112.56(12)$ & $C(43)-P(5)-C(37)$ & 104.47(18) \\
\hline$C(43)-P(5)-C(40)$ & $103.22(17)$ & $C(37)-P(5)-C(40)$ & $111.29(19)$ \\
\hline$C(43)-P(5)-R h(5)$ & $112.31(13)$ & $\mathrm{C}(37)-\mathrm{P}(5)-\mathrm{Rh}(5)$ & 111.71(12) \\
\hline $\mathrm{C}(40)-\mathrm{P}(5)-\operatorname{Rh}(5)$ & $113.21(13)$ & $C(52)-P(6)-C(49)$ & 104.12(17) \\
\hline$C(52)-P(6)-C(46)$ & $103.60(16)$ & $C(49)-P(6)-C(46)$ & $111.66(16)$ \\
\hline$C(52)-P(6)-R h(6)$ & $115.28(11)$ & $\mathrm{C}(49)-\mathrm{P}(6)-\mathrm{Rh}(6)$ & 109.67(11) \\
\hline $\mathrm{C}(46)-\mathrm{P}(6)-\operatorname{Rh}(6)$ & $112.17(11)$ & $C(2)-C(1)-C(3)$ & $110.9(8)$ \\
\hline$C(2)-C(1)-P(1)$ & $118.4(5)$ & $C(3)-C(1)-P(1)$ & $110.4(7)$ \\
\hline$C(5)-C(4)-C(6)$ & $115.9(14)$ & $C(5)-C(4)-P(1)$ & $117.7(11)$ \\
\hline $\mathrm{C}(6)-\mathrm{C}(4)-\mathrm{P}(1)$ & 111.1(9) & $\mathrm{B}(91)-\mathrm{C}(05)-\mathrm{B}(90)$ & $68.7(4)$ \\
\hline$B(91)-C(05)-B(86)$ & $60.7(5)$ & $\mathrm{B}(90)-\mathrm{C}(05)-\mathrm{B}(86)$ & $117.4(7)$ \\
\hline $\mathrm{B}(91)-\mathrm{C}(05)-\mathrm{B}(84)$ & $118.5(5)$ & $\mathrm{B}(90)-\mathrm{C}(05)-\mathrm{B}(84)$ & $63.0(4)$ \\
\hline $\mathrm{B}(86)-\mathrm{C}(05)-\mathrm{B}(84)$ & $113.0(6)$ & $\mathrm{B}(91)-\mathrm{C}(05)-\mathrm{B}(81)$ & $114.2(5)$ \\
\hline$B(90)-C(05)-B(81)$ & $112.3(5)$ & $\mathrm{B}(86)-\mathrm{C}(05)-\mathrm{B}(81)$ & $63.8(5)$ \\
\hline $\mathrm{B}(84)-\mathrm{C}(05)-\mathrm{B}(81)$ & $58.4(3)$ & $\mathrm{B}(81)-\mathrm{C}(06)-\mathrm{B}(82)$ & $65.8(5)$ \\
\hline $\mathrm{B}(81)-\mathrm{C}(06)-\mathrm{B}(85)$ & $57.6(5)$ & $\mathrm{B}(82)-\mathrm{C}(06)-\mathrm{B}(85)$ & $112.3(7)$ \\
\hline $\mathrm{B}(81)-\mathrm{C}(06)-\mathrm{B}(87)$ & $115.8(6)$ & $\mathrm{B}(82)-\mathrm{C}(06)-\mathrm{B}(87)$ & $62.9(4)$ \\
\hline $\mathrm{B}(85)-\mathrm{C}(06)-\mathrm{B}(87)$ & $112.6(7)$ & $\mathrm{B}(81)-\mathrm{C}(06)-\mathrm{B}(91)$ & $112.8(6)$ \\
\hline $\mathrm{B}(82)-\mathrm{C}(06)-\mathrm{B}(91)$ & $111.5(6)$ & $\mathrm{B}(85)-\mathrm{C}(06)-\mathrm{B}(91)$ & $65.4(5)$ \\
\hline $\mathrm{B}(87)-\mathrm{C}(06)-\mathrm{B}(91)$ & $58.4(4)$ & $\mathrm{C}(8)-\mathrm{C}(7)-\mathrm{C}(9)$ & 112.1(12) \\
\hline$C(8)-C(7)-P(1)$ & $114.3(13)$ & $\mathrm{C}(9)-\mathrm{C}(7)-\mathrm{P}(1)$ & $113.4(8)$ \\
\hline$C(12)-C(10)-C(11)$ & $110.3(4)$ & $\mathrm{C}(12)-\mathrm{C}(10)-\mathrm{P}(2)$ & $111.3(3)$ \\
\hline$C(11)-C(10)-P(2)$ & 115.9(3) & $\mathrm{C}(14)-\mathrm{C}(13)-\mathrm{C}(15)$ & 109.5(3) \\
\hline$C(14)-C(13)-P(2)$ & 114.1(3) & $C(15)-C(13)-P(2)$ & $114.9(3)$ \\
\hline $\mathrm{C}(17)-\mathrm{C}(16)-\mathrm{C}(18)$ & $111.1(3)$ & $\mathrm{C}(17)-\mathrm{C}(16)-\mathrm{P}(2)$ & 111.1(3) \\
\hline$C(18)-C(16)-P(2)$ & 111.1(3) & $\mathrm{C}(21)-\mathrm{C}(19)-\mathrm{C}(20)$ & $110.4(4)$ \\
\hline$C(21)-C(19)-P(3)$ & $112.9(3)$ & $C(20)-C(19)-P(3)$ & 111.3(3) \\
\hline$C(24)-C(22)-C(23)$ & 109.7(3) & $\mathrm{C}(24)-\mathrm{C}(22)-\mathrm{P}(3)$ & 114.7(3) \\
\hline$C(23)-C(22)-P(3)$ & $113.5(2)$ & $\mathrm{C}(26)-\mathrm{C}(25)-\mathrm{C}(27)$ & 109.5(4) \\
\hline$C(26)-C(25)-P(3)$ & $110.8(3)$ & $C(27)-C(25)-P(3)$ & $117.8(3)$ \\
\hline $\mathrm{C}(30)-\mathrm{C}(28)-\mathrm{C}(29)$ & $111.0(3)$ & $\mathrm{C}(30)-\mathrm{C}(28)-\mathrm{P}(4)$ & 113.4(3) \\
\hline$C(29)-C(28)-P(4)$ & $114.6(3)$ & $C(33)-C(31)-C(32)$ & 110.1(3) \\
\hline$C(33)-C(31)-P(4)$ & $112.7(3)$ & $\mathrm{C}(32)-\mathrm{C}(31)-\mathrm{P}(4)$ & 116.6(3) \\
\hline$C(36)-C(34)-C(35)$ & 110.6(3) & $\mathrm{C}(36)-\mathrm{C}(34)-\mathrm{P}(4)$ & 112.2(3) \\
\hline$C(35)-C(34)-P(4)$ & 110.9(3) & $\mathrm{C}(39)-\mathrm{C}(37)-\mathrm{C}(38)$ & 110.4(3) \\
\hline$C(39)-C(37)-P(5)$ & $112.7(3)$ & $C(38)-C(37)-P(5)$ & 115.7(3) \\
\hline $\mathrm{C}(41)-\mathrm{C}(40)-\mathrm{C}(42)$ & 109.8(4) & $\mathrm{C}(41)-\mathrm{C}(40)-\mathrm{P}(5)$ & $114.5(3)$ \\
\hline$C(42)-C(40)-P(5)$ & 115.2(3) & $C(45)-C(43)-C(44)$ & 111.1(3) \\
\hline$C(45)-C(43)-P(5)$ & $111.9(3)$ & $C(44)-C(43)-P(5)$ & $111.8(2)$ \\
\hline $\mathrm{C}(48)-\mathrm{C}(46)-\mathrm{C}(47)$ & 110.6(3) & $\mathrm{C}(48)-\mathrm{C}(46)-\mathrm{P}(6)$ & 114.1(2) \\
\hline $\mathrm{C}(47)-\mathrm{C}(46)-\mathrm{P}(6)$ & 115.5(3) & $C(51)-C(49)-C(50)$ & $110.5(3)$ \\
\hline$C(51)-C(49)-P(6)$ & $113.2(3)$ & $\mathrm{C}(50)-\mathrm{C}(49)-\mathrm{P}(6)$ & $115.4(3)$ \\
\hline$C(53)-C(52)-C(54)$ & $111.0(3)$ & $C(53)-C(52)-P(6)$ & $112.2(3)$ \\
\hline$C(54)-C(52)-P(6)$ & 111.9(3) & $\mathrm{B}(64)-\mathrm{C}(61)-\mathrm{B}(65)$ & $63.0(2)$ \\
\hline $\mathrm{B}(64)-\mathrm{C}(61)-\mathrm{B}(63)$ & $62.8(2)$ & $\mathrm{B}(65)-\mathrm{C}(61)-\mathrm{B}(63)$ & $115.1(3)$ \\
\hline
\end{tabular}




\begin{tabular}{|c|c|c|c|}
\hline $\mathrm{B}(64)-\mathrm{C}(61)-\mathrm{B}(62)$ & $115.3(3)$ & $\mathrm{B}(65)-\mathrm{C}(61)-\mathrm{B}(62)$ & $115.2(3)$ \\
\hline $\mathrm{B}(63)-\mathrm{C}(61)-\mathrm{B}(62)$ & $63.0(2)$ & $\mathrm{B}(64)-\mathrm{C}(61)-\mathrm{B}(66)$ & $115.6(3)$ \\
\hline $\mathrm{B}(65)-\mathrm{C}(61)-\mathrm{B}(66)$ & $63.1(3)$ & $\mathrm{B}(63)-\mathrm{C}(61)-\mathrm{B}(66)$ & $115.4(3)$ \\
\hline $\mathrm{B}(62)-\mathrm{C}(61)-\mathrm{B}(66)$ & $62.9(3)$ & $\mathrm{C}(902)-\mathrm{C}(901)-\mathrm{C}(903)$ & $43.7(10)$ \\
\hline $\mathrm{C}(903)-\mathrm{C}(902)-\mathrm{C}(901)$ & $89.3(15)$ & $\mathrm{C}(904)-\mathrm{C}(903)-\mathrm{C}(902)$ & $145.0(15)$ \\
\hline $\mathrm{C}(904)-\mathrm{C}(903)-\mathrm{C}(905)$ & $31.9(7)$ & $\mathrm{C}(902)-\mathrm{C}(903)-\mathrm{C}(905)$ & $125.4(14)$ \\
\hline $\mathrm{C}(904)-\mathrm{C}(903)-\mathrm{C}(901)$ & $120.0(13)$ & $\mathrm{C}(902)-\mathrm{C}(903)-\mathrm{C}(901)$ & $47.1(10)$ \\
\hline$C(905)-C(903)-C(901)$ & $88.5(12)$ & $C(905)-C(904)-C(903)$ & $89.3(17)$ \\
\hline $\mathrm{C}(904)-\mathrm{C}(905)-\mathrm{C}(903)$ & $58.8(14)$ & $C(906)-C(907)-C(909) \# 1$ & 102(2) \\
\hline $\mathrm{C}(908)-\mathrm{C}(909)-\mathrm{C}(907) \# 1$ & $116.6(17)$ & $C(62)-B(62)-C(61)$ & $120.7(3)$ \\
\hline$C(62)-B(62)-B(70)$ & $125.3(4)$ & $\mathrm{C}(61)-\mathrm{B}(62)-\mathrm{B}(70)$ & $104.4(3)$ \\
\hline$C(62)-B(62)-B(71)$ & $126.1(3)$ & $\mathrm{C}(61)-\mathrm{B}(62)-\mathrm{B}(71)$ & $104.4(3)$ \\
\hline $\mathrm{B}(70)-\mathrm{B}(62)-\mathrm{B}(71)$ & $60.9(2)$ & $\mathrm{C}(62)-\mathrm{B}(62)-\mathrm{B}(66)$ & $120.1(4)$ \\
\hline$C(61)-B(62)-B(66)$ & $58.6(2)$ & $\mathrm{B}(70)-\mathrm{B}(62)-\mathrm{B}(66)$ & $108.8(3)$ \\
\hline $\mathrm{B}(71)-\mathrm{B}(62)-\mathrm{B}(66)$ & $60.0(2)$ & $\mathrm{C}(62)-\mathrm{B}(62)-\mathrm{B}(63)$ & $119.5(3)$ \\
\hline $\mathrm{C}(61)-\mathrm{B}(62)-\mathrm{B}(63)$ & $58.5(2)$ & $\mathrm{B}(70)-\mathrm{B}(62)-\mathrm{B}(63)$ & $59.7(2)$ \\
\hline $\mathrm{B}(71)-\mathrm{B}(62)-\mathrm{B}(63)$ & 108.3(3) & $\mathrm{B}(66)-\mathrm{B}(62)-\mathrm{B}(63)$ & 108.1(3) \\
\hline$C(63)-B(63)-C(61)$ & $121.2(3)$ & $\mathrm{C}(63)-\mathrm{B}(63)-\mathrm{B}(69)$ & $125.3(3)$ \\
\hline$C(61)-B(63)-B(69)$ & 104.1(3) & $\mathrm{C}(63)-\mathrm{B}(63)-\mathrm{B}(70)$ & $125.4(3)$ \\
\hline$C(61)-B(63)-B(70)$ & 104.3(3) & $\mathrm{B}(69)-\mathrm{B}(63)-\mathrm{B}(70)$ & $61.0(2)$ \\
\hline$C(63)-B(63)-B(64)$ & $119.8(3)$ & $\mathrm{C}(61)-\mathrm{B}(63)-\mathrm{B}(64)$ & $58.2(2)$ \\
\hline $\mathrm{B}(69)-\mathrm{B}(63)-\mathrm{B}(64)$ & $60.0(2)$ & $\mathrm{B}(70)-\mathrm{B}(63)-\mathrm{B}(64)$ & $108.8(3)$ \\
\hline$C(63)-B(63)-B(62)$ & $120.5(3)$ & $\mathrm{C}(61)-\mathrm{B}(63)-\mathrm{B}(62)$ & $58.5(2)$ \\
\hline $\mathrm{B}(69)-\mathrm{B}(63)-\mathrm{B}(62)$ & $108.1(3)$ & $\mathrm{B}(70)-\mathrm{B}(63)-\mathrm{B}(62)$ & $59.5(2)$ \\
\hline $\mathrm{B}(64)-\mathrm{B}(63)-\mathrm{B}(62)$ & $107.8(3)$ & $\mathrm{C}(64)-\mathrm{B}(64)-\mathrm{C}(61)$ & $120.8(3)$ \\
\hline $\mathrm{C}(64)-\mathrm{B}(64)-\mathrm{B}(69)$ & 125.2(3) & $\mathrm{C}(61)-\mathrm{B}(64)-\mathrm{B}(69)$ & 104.5(3) \\
\hline $\mathrm{C}(64)-\mathrm{B}(64)-\mathrm{B}(63)$ & 119.2(3) & $\mathrm{C}(61)-\mathrm{B}(64)-\mathrm{B}(63)$ & $59.0(2)$ \\
\hline $\mathrm{B}(69)-\mathrm{B}(64)-\mathrm{B}(63)$ & $59.7(2)$ & $\mathrm{C}(64)-\mathrm{B}(64)-\mathrm{B}(65)$ & $120.7(3)$ \\
\hline $\mathrm{C}(61)-\mathrm{B}(64)-\mathrm{B}(65)$ & $58.7(2)$ & $\mathrm{B}(69)-\mathrm{B}(64)-\mathrm{B}(65)$ & 108.2(3) \\
\hline $\mathrm{B}(63)-\mathrm{B}(64)-\mathrm{B}(65)$ & 108.4(3) & $\mathrm{C}(64)-\mathrm{B}(64)-\mathrm{B}(68)$ & $125.8(3)$ \\
\hline $\mathrm{C}(61)-\mathrm{B}(64)-\mathrm{B}(68)$ & 104.6(3) & $\mathrm{B}(69)-\mathrm{B}(64)-\mathrm{B}(68)$ & $60.5(2)$ \\
\hline $\mathrm{B}(63)-\mathrm{B}(64)-\mathrm{B}(68)$ & $108.5(3)$ & $\mathrm{B}(65)-\mathrm{B}(64)-\mathrm{B}(68)$ & $59.9(2)$ \\
\hline $\mathrm{C}(65)-\mathrm{B}(65)-\mathrm{C}(61)$ & $120.7(3)$ & $\mathrm{C}(65)-\mathrm{B}(65)-\mathrm{B}(68)$ & $125.6(4)$ \\
\hline $\mathrm{C}(61)-\mathrm{B}(65)-\mathrm{B}(68)$ & 104.6(3) & $\mathrm{C}(65)-\mathrm{B}(65)-\mathrm{B}(67)$ & $125.5(3)$ \\
\hline $\mathrm{C}(61)-\mathrm{B}(65)-\mathrm{B}(67)$ & $104.5(3)$ & $\mathrm{B}(68)-\mathrm{B}(65)-\mathrm{B}(67)$ & $60.8(2)$ \\
\hline $\mathrm{C}(65)-\mathrm{B}(65)-\mathrm{B}(64)$ & 120.1(3) & $\mathrm{C}(61)-\mathrm{B}(65)-\mathrm{B}(64)$ & $58.4(2)$ \\
\hline $\mathrm{B}(68)-\mathrm{B}(65)-\mathrm{B}(64)$ & $60.1(2)$ & $\mathrm{B}(67)-\mathrm{B}(65)-\mathrm{B}(64)$ & 108.6(3) \\
\hline $\mathrm{C}(65)-\mathrm{B}(65)-\mathrm{B}(66)$ & 119.5(3) & $\mathrm{C}(61)-\mathrm{B}(65)-\mathrm{B}(66)$ & $58.8(2)$ \\
\hline $\mathrm{B}(68)-\mathrm{B}(65)-\mathrm{B}(66)$ & 108.7(3) & $\mathrm{B}(67)-\mathrm{B}(65)-\mathrm{B}(66)$ & $59.9(2)$ \\
\hline $\mathrm{B}(64)-\mathrm{B}(65)-\mathrm{B}(66)$ & 108.2(3) & $\mathrm{C}(66)-\mathrm{B}(66)-\mathrm{C}(61)$ & $121.6(3)$ \\
\hline $\mathrm{C}(66)-\mathrm{B}(66)-\mathrm{B}(67)$ & $125.4(4)$ & $\mathrm{C}(61)-\mathrm{B}(66)-\mathrm{B}(67)$ & $103.9(3)$ \\
\hline $\mathrm{C}(66)-\mathrm{B}(66)-\mathrm{B}(71)$ & $125.5(3)$ & $\mathrm{C}(61)-\mathrm{B}(66)-\mathrm{B}(71)$ & $103.9(3)$ \\
\hline $\mathrm{B}(67)-\mathrm{B}(66)-\mathrm{B}(71)$ & $60.5(2)$ & $\mathrm{C}(66)-\mathrm{B}(66)-\mathrm{B}(62)$ & $120.4(4)$ \\
\hline $\mathrm{C}(61)-\mathrm{B}(66)-\mathrm{B}(62)$ & $58.5(2)$ & $\mathrm{B}(67)-\mathrm{B}(66)-\mathrm{B}(62)$ & $107.9(3)$ \\
\hline $\mathrm{B}(71)-\mathrm{B}(66)-\mathrm{B}(62)$ & $59.5(2)$ & $\mathrm{C}(66)-\mathrm{B}(66)-\mathrm{B}(65)$ & $120.5(4)$ \\
\hline $\mathrm{C}(61)-\mathrm{B}(66)-\mathrm{B}(65)$ & $58.2(2)$ & $\mathrm{B}(67)-\mathrm{B}(66)-\mathrm{B}(65)$ & $59.7(2)$ \\
\hline $\mathrm{B}(71)-\mathrm{B}(66)-\mathrm{B}(65)$ & $107.9(3)$ & $\mathrm{B}(62)-\mathrm{B}(66)-\mathrm{B}(65)$ & 107.5(3) \\
\hline $\mathrm{C}(67)-\mathrm{B}(67)-\mathrm{B}(65)$ & $120.5(3)$ & $\mathrm{C}(67)-\mathrm{B}(67)-\mathrm{B}(66)$ & $120.8(3)$ \\
\hline $\mathrm{B}(65)-\mathrm{B}(67)-\mathrm{B}(66)$ & $60.4(2)$ & $\mathrm{C}(67)-\mathrm{B}(67)-\mathrm{B}(72)$ & $123.5(3)$ \\
\hline $\mathrm{B}(65)-\mathrm{B}(67)-\mathrm{B}(72)$ & 107.3(3) & $\mathrm{B}(66)-\mathrm{B}(67)-\mathrm{B}(72)$ & $107.7(3)$ \\
\hline $\mathrm{C}(67)-\mathrm{B}(67)-\mathrm{B}(71)$ & $122.8(3)$ & $\mathrm{B}(65)-\mathrm{B}(67)-\mathrm{B}(71)$ & $107.9(3)$ \\
\hline $\mathrm{B}(66)-\mathrm{B}(67)-\mathrm{B}(71)$ & $59.8(2)$ & $\mathrm{B}(72)-\mathrm{B}(67)-\mathrm{B}(71)$ & $59.9(2)$ \\
\hline $\mathrm{C}(67)-\mathrm{B}(67)-\mathrm{B}(68)$ & $121.6(4)$ & $\mathrm{B}(65)-\mathrm{B}(67)-\mathrm{B}(68)$ & $59.5(2)$ \\
\hline $\mathrm{B}(66)-\mathrm{B}(67)-\mathrm{B}(68)$ & 108.2(3) & $\mathrm{B}(72)-\mathrm{B}(67)-\mathrm{B}(68)$ & $60.0(2)$ \\
\hline
\end{tabular}




\begin{tabular}{|c|c|c|c|}
\hline $\mathrm{B}(71)-\mathrm{B}(67)-\mathrm{B}(68)$ & 108.0(3) & $\mathrm{C}(68)-\mathrm{B}(68)-\mathrm{B}(65)$ & 121.3(3) \\
\hline $\mathrm{C}(68)-\mathrm{B}(68)-\mathrm{B}(64)$ & 120.9(3) & $\mathrm{B}(65)-\mathrm{B}(68)-\mathrm{B}(64)$ & $60.0(2)$ \\
\hline $\mathrm{C}(68)-\mathrm{B}(68)-\mathrm{B}(69)$ & $122.0(3)$ & $\mathrm{B}(65)-\mathrm{B}(68)-\mathrm{B}(69)$ & 107.6(3) \\
\hline $\mathrm{B}(64)-\mathrm{B}(68)-\mathrm{B}(69)$ & $59.6(2)$ & $\mathrm{C}(68)-\mathrm{B}(68)-\mathrm{B}(72)$ & 123.3(3) \\
\hline $\mathrm{B}(65)-\mathrm{B}(68)-\mathrm{B}(72)$ & 107.3(3) & $\mathrm{B}(64)-\mathrm{B}(68)-\mathrm{B}(72)$ & $107.2(3)$ \\
\hline $\mathrm{B}(69)-\mathrm{B}(68)-\mathrm{B}(72)$ & $59.8(2)$ & $\mathrm{C}(68)-\mathrm{B}(68)-\mathrm{B}(67)$ & $122.6(3)$ \\
\hline $\mathrm{B}(65)-\mathrm{B}(68)-\mathrm{B}(67)$ & $59.6(2)$ & $\mathrm{B}(64)-\mathrm{B}(68)-\mathrm{B}(67)$ & $107.6(3)$ \\
\hline $\mathrm{B}(69)-\mathrm{B}(68)-\mathrm{B}(67)$ & $107.7(3)$ & $\mathrm{B}(72)-\mathrm{B}(68)-\mathrm{B}(67)$ & $59.9(2)$ \\
\hline $\mathrm{C}(69)-\mathrm{B}(69)-\mathrm{B}(63)$ & $121.2(3)$ & $\mathrm{C}(69)-\mathrm{B}(69)-\mathrm{B}(64)$ & $120.5(3)$ \\
\hline $\mathrm{B}(63)-\mathrm{B}(69)-\mathrm{B}(64)$ & $60.3(2)$ & $\mathrm{C}(69)-\mathrm{B}(69)-\mathrm{B}(72)$ & $122.8(3)$ \\
\hline $\mathrm{B}(63)-\mathrm{B}(69)-\mathrm{B}(72)$ & $107.9(3)$ & $\mathrm{B}(64)-\mathrm{B}(69)-\mathrm{B}(72)$ & $107.9(3)$ \\
\hline $\mathrm{C}(69)-\mathrm{B}(69)-\mathrm{B}(68)$ & $120.9(3)$ & $\mathrm{B}(63)-\mathrm{B}(69)-\mathrm{B}(68)$ & $108.4(3)$ \\
\hline $\mathrm{B}(64)-\mathrm{B}(69)-\mathrm{B}(68)$ & $59.9(2)$ & $\mathrm{B}(72)-\mathrm{B}(69)-\mathrm{B}(68)$ & $60.3(2)$ \\
\hline $\mathrm{C}(69)-\mathrm{B}(69)-\mathrm{B}(70)$ & $122.8(3)$ & $\mathrm{B}(63)-\mathrm{B}(69)-\mathrm{B}(70)$ & $59.6(2)$ \\
\hline $\mathrm{B}(64)-\mathrm{B}(69)-\mathrm{B}(70)$ & $107.8(3)$ & $\mathrm{B}(72)-\mathrm{B}(69)-\mathrm{B}(70)$ & $60.2(2)$ \\
\hline $\mathrm{B}(68)-\mathrm{B}(69)-\mathrm{B}(70)$ & $108.5(3)$ & $\mathrm{C}(70)-\mathrm{B}(70)-\mathrm{B}(62)$ & $120.6(3)$ \\
\hline $\mathrm{C}(70)-\mathrm{B}(70)-\mathrm{B}(63)$ & $120.9(3)$ & $\mathrm{B}(62)-\mathrm{B}(70)-\mathrm{B}(63)$ & $60.8(2)$ \\
\hline $\mathrm{C}(70)-\mathrm{B}(70)-\mathrm{B}(71)$ & $122.0(3)$ & $\mathrm{B}(62)-\mathrm{B}(70)-\mathrm{B}(71)$ & $59.7(2)$ \\
\hline $\mathrm{B}(63)-\mathrm{B}(70)-\mathrm{B}(71)$ & $108.2(3)$ & $\mathrm{C}(70)-\mathrm{B}(70)-\mathrm{B}(72)$ & $123.4(3)$ \\
\hline $\mathrm{B}(62)-\mathrm{B}(70)-\mathrm{B}(72)$ & $107.5(3)$ & $\mathrm{B}(63)-\mathrm{B}(70)-\mathrm{B}(72)$ & $107.3(3)$ \\
\hline $\mathrm{B}(71)-\mathrm{B}(70)-\mathrm{B}(72)$ & $59.8(2)$ & $\mathrm{C}(70)-\mathrm{B}(70)-\mathrm{B}(69)$ & $122.6(3)$ \\
\hline $\mathrm{B}(62)-\mathrm{B}(70)-\mathrm{B}(69)$ & $107.9(3)$ & $\mathrm{B}(63)-\mathrm{B}(70)-\mathrm{B}(69)$ & $59.4(2)$ \\
\hline $\mathrm{B}(71)-\mathrm{B}(70)-\mathrm{B}(69)$ & $107.6(3)$ & $\mathrm{B}(72)-\mathrm{B}(70)-\mathrm{B}(69)$ & $59.6(2)$ \\
\hline $\mathrm{C}(107)-\mathrm{B}(71)-\mathrm{B}(62)$ & $121.6(3)$ & $\mathrm{C}(107)-\mathrm{B}(71)-\mathrm{B}(66)$ & 122.1(3) \\
\hline $\mathrm{B}(62)-\mathrm{B}(71)-\mathrm{B}(66)$ & $60.5(3)$ & $\mathrm{C}(107)-\mathrm{B}(71)-\mathrm{B}(72)$ & $121.8(3)$ \\
\hline $\mathrm{B}(62)-\mathrm{B}(71)-\mathrm{B}(72)$ & 107.6(3) & $\mathrm{B}(66)-\mathrm{B}(71)-\mathrm{B}(72)$ & $107.7(3)$ \\
\hline $\mathrm{C}(107)-\mathrm{B}(71)-\mathrm{B}(70)$ & 121.1(3) & $\mathrm{B}(62)-\mathrm{B}(71)-\mathrm{B}(70)$ & $59.5(2)$ \\
\hline $\mathrm{B}(66)-\mathrm{B}(71)-\mathrm{B}(70)$ & 108.1(3) & $\mathrm{B}(72)-\mathrm{B}(71)-\mathrm{B}(70)$ & $60.2(2)$ \\
\hline $\mathrm{C}(107)-\mathrm{B}(71)-\mathrm{B}(67)$ & $122.2(3)$ & $\mathrm{B}(62)-\mathrm{B}(71)-\mathrm{B}(67)$ & $108.0(3)$ \\
\hline $\mathrm{B}(66)-\mathrm{B}(71)-\mathrm{B}(67)$ & $59.7(3)$ & $\mathrm{B}(72)-\mathrm{B}(71)-\mathrm{B}(67)$ & $60.0(2)$ \\
\hline $\mathrm{B}(70)-\mathrm{B}(71)-\mathrm{B}(67)$ & $108.2(3)$ & $\mathrm{C}(71)-\mathrm{B}(72)-\mathrm{B}(69)$ & $121.8(3)$ \\
\hline $\mathrm{C}(71)-\mathrm{B}(72)-\mathrm{B}(71)$ & $121.2(3)$ & $\mathrm{B}(69)-\mathrm{B}(72)-\mathrm{B}(71)$ & 108.2(3) \\
\hline $\mathrm{C}(71)-\mathrm{B}(72)-\mathrm{B}(67)$ & $121.8(3)$ & $\mathrm{B}(69)-\mathrm{B}(72)-\mathrm{B}(67)$ & $108.0(3)$ \\
\hline $\mathrm{B}(71)-\mathrm{B}(72)-\mathrm{B}(67)$ & $60.1(2)$ & $\mathrm{C}(71)-\mathrm{B}(72)-\mathrm{B}(68)$ & $122.0(3)$ \\
\hline $\mathrm{B}(69)-\mathrm{B}(72)-\mathrm{B}(68)$ & $60.0(2)$ & $\mathrm{B}(71)-\mathrm{B}(72)-\mathrm{B}(68)$ & 108.3(3) \\
\hline $\mathrm{B}(67)-\mathrm{B}(72)-\mathrm{B}(68)$ & $60.1(2)$ & $\mathrm{C}(71)-\mathrm{B}(72)-\mathrm{B}(70)$ & $121.3(3)$ \\
\hline $\mathrm{B}(69)-\mathrm{B}(72)-\mathrm{B}(70)$ & $60.2(2)$ & $\mathrm{B}(71)-\mathrm{B}(72)-\mathrm{B}(70)$ & $60.0(2)$ \\
\hline $\mathrm{B}(67)-\mathrm{B}(72)-\mathrm{B}(70)$ & 108.1(3) & $\mathrm{B}(68)-\mathrm{B}(72)-\mathrm{B}(70)$ & 108.3(3) \\
\hline $\mathrm{C}(81)-\mathrm{B}(81)-\mathrm{B}(85)$ & 115.4(6) & $\mathrm{C}(81)-\mathrm{B}(81)-\mathrm{C}(06)$ & $120.5(5)$ \\
\hline $\mathrm{B}(85)-\mathrm{B}(81)-\mathrm{C}(06)$ & $64.0(6)$ & $\mathrm{C}(81)-\mathrm{B}(81)-\mathrm{B}(82)$ & 124.1(4) \\
\hline $\mathrm{B}(85)-\mathrm{B}(81)-\mathrm{B}(82)$ & 110.9(5) & $\mathrm{C}(06)-\mathrm{B}(81)-\mathrm{B}(82)$ & $57.7(4)$ \\
\hline $\mathrm{C}(81)-\mathrm{B}(81)-\mathrm{B}(83)$ & $125.6(4)$ & $\mathrm{B}(85)-\mathrm{B}(81)-\mathrm{B}(83)$ & $108.9(5)$ \\
\hline $\mathrm{C}(06)-\mathrm{B}(81)-\mathrm{B}(83)$ & $106.3(5)$ & $\mathrm{B}(82)-\mathrm{B}(81)-\mathrm{B}(83)$ & $60.5(2)$ \\
\hline $\mathrm{C}(81)-\mathrm{B}(81)-\mathrm{B}(84)$ & 121.3(3) & $\mathrm{B}(85)-\mathrm{B}(81)-\mathrm{B}(84)$ & $59.4(4)$ \\
\hline $\mathrm{C}(06)-\mathrm{B}(81)-\mathrm{B}(84)$ & 108.3(5) & $\mathrm{B}(82)-\mathrm{B}(81)-\mathrm{B}(84)$ & $108.2(3)$ \\
\hline $\mathrm{B}(83)-\mathrm{B}(81)-\mathrm{B}(84)$ & $59.7(2)$ & $\mathrm{C}(81)-\mathrm{B}(81)-\mathrm{C}(05)$ & $124.5(5)$ \\
\hline$B(85)-B(81)-C(05)$ & $9.6(5)$ & $\mathrm{C}(06)-\mathrm{B}(81)-\mathrm{C}(05)$ & $58.0(5)$ \\
\hline $\mathrm{B}(82)-\mathrm{B}(81)-\mathrm{C}(05)$ & 101.6(4) & $\mathrm{B}(83)-\mathrm{B}(81)-\mathrm{C}(05)$ & $102.5(4)$ \\
\hline $\mathrm{B}(84)-\mathrm{B}(81)-\mathrm{C}(05)$ & $58.9(3)$ & $\mathrm{C}(81)-\mathrm{B}(81)-\mathrm{B}(86)$ & $121.6(5)$ \\
\hline $\mathrm{B}(85)-\mathrm{B}(81)-\mathrm{B}(86)$ & $59.6(5)$ & $\mathrm{C}(06)-\mathrm{B}(81)-\mathrm{B}(86)$ & $4.4(6)$ \\
\hline $\mathrm{B}(82)-\mathrm{B}(81)-\mathrm{B}(86)$ & $61.1(4)$ & $\mathrm{B}(83)-\mathrm{B}(81)-\mathrm{B}(86)$ & $107.2(4)$ \\
\hline $\mathrm{B}(84)-\mathrm{B}(81)-\mathrm{B}(86)$ & $105.0(4)$ & $\mathrm{C}(05)-\mathrm{B}(81)-\mathrm{B}(86)$ & $53.7(4)$ \\
\hline $\mathrm{C}(82)-\mathrm{B}(82)-\mathrm{C}(06)$ & $121.0(5)$ & $\mathrm{C}(82)-\mathrm{B}(82)-\mathrm{B}(81)$ & $121.0(3)$ \\
\hline $\mathrm{C}(06)-\mathrm{B}(82)-\mathrm{B}(81)$ & $56.5(4)$ & $\mathrm{C}(82)-\mathrm{B}(82)-\mathrm{B}(88)$ & $123.8(3)$ \\
\hline
\end{tabular}




\begin{tabular}{|c|c|c|c|}
\hline $\mathrm{C}(06)-\mathrm{B}(82)-\mathrm{B}(88)$ & $108.0(4)$ & $\mathrm{B}(81)-\mathrm{B}(82)-\mathrm{B}(88)$ & 107.8(3) \\
\hline $\mathrm{C}(82)-\mathrm{B}(82)-\mathrm{B}(83)$ & $123.6(3)$ & $\mathrm{C}(06)-\mathrm{B}(82)-\mathrm{B}(83)$ & 104.8(4) \\
\hline $\mathrm{B}(81)-\mathrm{B}(82)-\mathrm{B}(83)$ & $59.9(2)$ & $\mathrm{B}(88)-\mathrm{B}(82)-\mathrm{B}(83)$ & $60.0(2)$ \\
\hline $\mathrm{C}(82)-\mathrm{B}(82)-\mathrm{B}(87)$ & $121.0(3)$ & $\mathrm{C}(06)-\mathrm{B}(82)-\mathrm{B}(87)$ & $62.5(4)$ \\
\hline $\mathrm{B}(81)-\mathrm{B}(82)-\mathrm{B}(87)$ & $108.1(3)$ & $\mathrm{B}(88)-\mathrm{B}(82)-\mathrm{B}(87)$ & $59.8(2)$ \\
\hline $\mathrm{B}(83)-\mathrm{B}(82)-\mathrm{B}(87)$ & $108.0(3)$ & $\mathrm{C}(82)-\mathrm{B}(82)-\mathrm{B}(86)$ & $121.5(5)$ \\
\hline $\mathrm{C}(06)-\mathrm{B}(82)-\mathrm{B}(86)$ & $6.6(6)$ & $\mathrm{B}(81)-\mathrm{B}(82)-\mathrm{B}(86)$ & $62.5(4)$ \\
\hline $\mathrm{B}(88)-\mathrm{B}(82)-\mathrm{B}(86)$ & 104.1(4) & $\mathrm{B}(83)-\mathrm{B}(82)-\mathrm{B}(86)$ & 107.8(4) \\
\hline $\mathrm{B}(87)-\mathrm{B}(82)-\mathrm{B}(86)$ & $56.1(4)$ & $\mathrm{C}(83)-\mathrm{B}(83)-\mathrm{B}(84)$ & 121.5(3) \\
\hline$C(83)-B(83)-B(81)$ & $120.8(4)$ & $\mathrm{B}(84)-\mathrm{B}(83)-\mathrm{B}(81)$ & $60.5(2)$ \\
\hline $\mathrm{C}(83)-\mathrm{B}(83)-\mathrm{B}(89)$ & $122.8(3)$ & $\mathrm{B}(84)-\mathrm{B}(83)-\mathrm{B}(89)$ & $59.9(2)$ \\
\hline $\mathrm{B}(81)-\mathrm{B}(83)-\mathrm{B}(89)$ & $108.0(3)$ & $\mathrm{C}(83)-\mathrm{B}(83)-\mathrm{B}(88)$ & $122.6(4)$ \\
\hline $\mathrm{B}(84)-\mathrm{B}(83)-\mathrm{B}(88)$ & 107.9(3) & $\mathrm{B}(81)-\mathrm{B}(83)-\mathrm{B}(88)$ & 107.5(3) \\
\hline $\mathrm{B}(89)-\mathrm{B}(83)-\mathrm{B}(88)$ & $60.0(2)$ & $\mathrm{C}(83)-\mathrm{B}(83)-\mathrm{B}(82)$ & 121.1(3) \\
\hline $\mathrm{B}(84)-\mathrm{B}(83)-\mathrm{B}(82)$ & $108.3(3)$ & $\mathrm{B}(81)-\mathrm{B}(83)-\mathrm{B}(82)$ & $59.7(2)$ \\
\hline $\mathrm{B}(89)-\mathrm{B}(83)-\mathrm{B}(82)$ & $107.9(3)$ & $\mathrm{B}(88)-\mathrm{B}(83)-\mathrm{B}(82)$ & $59.8(2)$ \\
\hline $\mathrm{C}(84)-\mathrm{B}(84)-\mathrm{B}(85)$ & 117.3(5) & $\mathrm{C}(84)-\mathrm{B}(84)-\mathrm{B}(83)$ & 124.8(4) \\
\hline $\mathrm{B}(85)-\mathrm{B}(84)-\mathrm{B}(83)$ & $105.2(4)$ & $\mathrm{C}(84)-\mathrm{B}(84)-\mathrm{B}(89)$ & $125.0(3)$ \\
\hline $\mathrm{B}(85)-\mathrm{B}(84)-\mathrm{B}(89)$ & $110.9(5)$ & $\mathrm{B}(83)-\mathrm{B}(84)-\mathrm{B}(89)$ & $60.4(2)$ \\
\hline $\mathrm{C}(84)-\mathrm{B}(84)-\mathrm{B}(90)$ & $120.5(4)$ & $\mathrm{B}(85)-\mathrm{B}(84)-\mathrm{B}(90)$ & $65.3(4)$ \\
\hline $\mathrm{B}(83)-\mathrm{B}(84)-\mathrm{B}(90)$ & 108.1(3) & $\mathrm{B}(89)-\mathrm{B}(84)-\mathrm{B}(90)$ & $59.7(2)$ \\
\hline $\mathrm{C}(84)-\mathrm{B}(84)-\mathrm{B}(81)$ & $120.7(3)$ & $\mathrm{B}(85)-\mathrm{B}(84)-\mathrm{B}(81)$ & $54.8(4)$ \\
\hline $\mathrm{B}(83)-\mathrm{B}(84)-\mathrm{B}(81)$ & $59.8(2)$ & $\mathrm{B}(89)-\mathrm{B}(84)-\mathrm{B}(81)$ & 107.8(3) \\
\hline $\mathrm{B}(90)-\mathrm{B}(84)-\mathrm{B}(81)$ & $107.8(3)$ & $\mathrm{C}(84)-\mathrm{B}(84)-\mathrm{C}(05)$ & $123.6(4)$ \\
\hline $\mathrm{B}(85)-\mathrm{B}(84)-\mathrm{C}(05)$ & $12.3(4)$ & $\mathrm{B}(83)-\mathrm{B}(84)-\mathrm{C}(05)$ & $105.4(4)$ \\
\hline $\mathrm{B}(89)-\mathrm{B}(84)-\mathrm{C}(05)$ & $100.0(4)$ & $\mathrm{B}(90)-\mathrm{B}(84)-\mathrm{C}(05)$ & $53.3(3)$ \\
\hline $\mathrm{B}(81)-\mathrm{B}(84)-\mathrm{C}(05)$ & $62.7(4)$ & $\mathrm{B}(81)-\mathrm{B}(85)-\mathrm{C}(85)$ & 118.1(9) \\
\hline $\mathrm{B}(81)-\mathrm{B}(85)-\mathrm{B}(84)$ & $65.8(5)$ & $\mathrm{C}(85)-\mathrm{B}(85)-\mathrm{B}(84)$ & $124.5(8)$ \\
\hline $\mathrm{B}(81)-\mathrm{B}(85)-\mathrm{C}(06)$ & $58.4(5)$ & $\mathrm{C}(85)-\mathrm{B}(85)-\mathrm{C}(06)$ & $118.8(8)$ \\
\hline $\mathrm{B}(84)-\mathrm{B}(85)-\mathrm{C}(06)$ & $109.0(7)$ & $\mathrm{B}(81)-\mathrm{B}(85)-\mathrm{B}(90)$ & $111.9(6)$ \\
\hline $\mathrm{C}(85)-\mathrm{B}(85)-\mathrm{B}(90)$ & $125.2(8)$ & $\mathrm{B}(84)-\mathrm{B}(85)-\mathrm{B}(90)$ & $59.9(4)$ \\
\hline $\mathrm{C}(06)-\mathrm{B}(85)-\mathrm{B}(90)$ & 105.1(7) & $\mathrm{B}(81)-\mathrm{B}(85)-\mathrm{B}(91)$ & 109.7(6) \\
\hline $\mathrm{C}(85)-\mathrm{B}(85)-\mathrm{B}(91)$ & $120.0(8)$ & $\mathrm{B}(84)-\mathrm{B}(85)-\mathrm{B}(91)$ & 106.5(6) \\
\hline $\mathrm{C}(06)-\mathrm{B}(85)-\mathrm{B}(91)$ & $60.9(5)$ & $\mathrm{B}(90)-\mathrm{B}(85)-\mathrm{B}(91)$ & $56.2(4)$ \\
\hline $\mathrm{C}(86)-\mathrm{B}(86)-\mathrm{B}(91)$ & $121.3(8)$ & $\mathrm{C}(86)-\mathrm{B}(86)-\mathrm{C}(05)$ & $120.9(8)$ \\
\hline $\mathrm{B}(91)-\mathrm{B}(86)-\mathrm{C}(05)$ & $55.9(5)$ & $\mathrm{C}(86)-\mathrm{B}(86)-\mathrm{B}(87)$ & $126.6(8)$ \\
\hline $\mathrm{B}(91)-\mathrm{B}(86)-\mathrm{B}(87)$ & $63.5(5)$ & $\mathrm{C}(05)-\mathrm{B}(86)-\mathrm{B}(87)$ & $104.9(7)$ \\
\hline $\mathrm{C}(86)-\mathrm{B}(86)-\mathrm{B}(82)$ & $124.0(8)$ & $\mathrm{B}(91)-\mathrm{B}(86)-\mathrm{B}(82)$ & 110.2(6) \\
\hline $\mathrm{C}(05)-\mathrm{B}(86)-\mathrm{B}(82)$ & $104.8(7)$ & $\mathrm{B}(87)-\mathrm{B}(86)-\mathrm{B}(82)$ & $59.9(4)$ \\
\hline $\mathrm{C}(86)-\mathrm{B}(86)-\mathrm{B}(81)$ & $118.0(7)$ & $\mathrm{B}(91)-\mathrm{B}(86)-\mathrm{B}(81)$ & 109.3(6) \\
\hline $\mathrm{C}(05)-\mathrm{B}(86)-\mathrm{B}(81)$ & $62.5(5)$ & $\mathrm{B}(87)-\mathrm{B}(86)-\mathrm{B}(81)$ & 106.2(6) \\
\hline $\mathrm{B}(82)-\mathrm{B}(86)-\mathrm{B}(81)$ & $56.4(4)$ & $\mathrm{C}(87)-\mathrm{B}(87)-\mathrm{B}(86)$ & $119.4(5)$ \\
\hline $\mathrm{C}(87)-\mathrm{B}(87)-\mathrm{B}(91)$ & $120.7(3)$ & $\mathrm{B}(86)-\mathrm{B}(87)-\mathrm{B}(91)$ & $56.4(4)$ \\
\hline $\mathrm{C}(87)-\mathrm{B}(87)-\mathrm{B}(88)$ & $123.6(3)$ & $\mathrm{B}(86)-\mathrm{B}(87)-\mathrm{B}(88)$ & 110.2(5) \\
\hline $\mathrm{B}(91)-\mathrm{B}(87)-\mathrm{B}(88)$ & 107.8(3) & $\mathrm{C}(87)-\mathrm{B}(87)-\mathrm{B}(92)$ & $122.7(3)$ \\
\hline $\mathrm{B}(86)-\mathrm{B}(87)-\mathrm{B}(92)$ & $106.2(5)$ & $\mathrm{B}(91)-\mathrm{B}(87)-\mathrm{B}(92)$ & $59.8(2)$ \\
\hline $\mathrm{B}(88)-\mathrm{B}(87)-\mathrm{B}(92)$ & $60.1(2)$ & $\mathrm{C}(87)-\mathrm{B}(87)-\mathrm{C}(06)$ & 124.4(5) \\
\hline $\mathrm{B}(86)-\mathrm{B}(87)-\mathrm{C}(06)$ & $9.6(6)$ & $\mathrm{B}(91)-\mathrm{B}(87)-\mathrm{C}(06)$ & $62.5(4)$ \\
\hline $\mathrm{B}(88)-\mathrm{B}(87)-\mathrm{C}(06)$ & 101.8(4) & $\mathrm{B}(92)-\mathrm{B}(87)-\mathrm{C}(06)$ & 106.4(4) \\
\hline $\mathrm{C}(87)-\mathrm{B}(87)-\mathrm{B}(82)$ & $121.8(3)$ & $\mathrm{B}(86)-\mathrm{B}(87)-\mathrm{B}(82)$ & $64.0(5)$ \\
\hline $\mathrm{B}(91)-\mathrm{B}(87)-\mathrm{B}(82)$ & $108.0(3)$ & $\mathrm{B}(88)-\mathrm{B}(87)-\mathrm{B}(82)$ & $59.7(2)$ \\
\hline $\mathrm{B}(92)-\mathrm{B}(87)-\mathrm{B}(82)$ & $108.0(3)$ & $\mathrm{C}(06)-\mathrm{B}(87)-\mathrm{B}(82)$ & $54.6(4)$ \\
\hline $\mathrm{C}(88)-\mathrm{B}(88)-\mathrm{B}(82)$ & 120.6(3) & $\mathrm{C}(88)-\mathrm{B}(88)-\mathrm{B}(87)$ & $121.0(3)$ \\
\hline $\mathrm{B}(82)-\mathrm{B}(88)-\mathrm{B}(87)$ & $60.5(2)$ & $\mathrm{C}(88)-\mathrm{B}(88)-\mathrm{B}(89)$ & $122.4(3)$ \\
\hline
\end{tabular}




\begin{tabular}{|c|c|c|c|}
\hline $\mathrm{B}(82)-\mathrm{B}(88)-\mathrm{B}(89)$ & $108.2(3)$ & $\mathrm{B}(87)-\mathrm{B}(88)-\mathrm{B}(89)$ & $108.2(3)$ \\
\hline $\mathrm{C}(88)-\mathrm{B}(88)-\mathrm{B}(83)$ & $121.4(3)$ & $\mathrm{B}(82)-\mathrm{B}(88)-\mathrm{B}(83)$ & $60.1(2)$ \\
\hline $\mathrm{B}(87)-\mathrm{B}(88)-\mathrm{B}(83)$ & 108.6(3) & $\mathrm{B}(89)-\mathrm{B}(88)-\mathrm{B}(83)$ & $60.0(2)$ \\
\hline $\mathrm{C}(88)-\mathrm{B}(88)-\mathrm{B}(92)$ & $122.0(3)$ & $\mathrm{B}(82)-\mathrm{B}(88)-\mathrm{B}(92)$ & $108.5(3)$ \\
\hline $\mathrm{B}(87)-\mathrm{B}(88)-\mathrm{B}(92)$ & $60.0(2)$ & $\mathrm{B}(89)-\mathrm{B}(88)-\mathrm{B}(92)$ & $60.1(2)$ \\
\hline $\mathrm{B}(83)-\mathrm{B}(88)-\mathrm{B}(92)$ & $108.4(3)$ & $\mathrm{C}(89)-\mathrm{B}(89)-\mathrm{B}(90)$ & $120.8(3)$ \\
\hline $\mathrm{C}(89)-\mathrm{B}(89)-\mathrm{B}(84)$ & $121.3(3)$ & $\mathrm{B}(90)-\mathrm{B}(89)-\mathrm{B}(84)$ & $60.5(3)$ \\
\hline $\mathrm{C}(89)-\mathrm{B}(89)-\mathrm{B}(88)$ & $122.4(3)$ & $\mathrm{B}(90)-\mathrm{B}(89)-\mathrm{B}(88)$ & $108.0(3)$ \\
\hline $\mathrm{B}(84)-\mathrm{B}(89)-\mathrm{B}(88)$ & $107.9(3)$ & $\mathrm{C}(89)-\mathrm{B}(89)-\mathrm{B}(83)$ & $122.2(3)$ \\
\hline $\mathrm{B}(90)-\mathrm{B}(89)-\mathrm{B}(83)$ & $108.3(3)$ & $\mathrm{B}(84)-\mathrm{B}(89)-\mathrm{B}(83)$ & $59.8(2)$ \\
\hline $\mathrm{B}(88)-\mathrm{B}(89)-\mathrm{B}(83)$ & $60.0(2)$ & $\mathrm{C}(89)-\mathrm{B}(89)-\mathrm{B}(92)$ & $121.2(3)$ \\
\hline $\mathrm{B}(90)-\mathrm{B}(89)-\mathrm{B}(92)$ & $60.0(2)$ & $\mathrm{B}(84)-\mathrm{B}(89)-\mathrm{B}(92)$ & $108.6(3)$ \\
\hline $\mathrm{B}(88)-\mathrm{B}(89)-\mathrm{B}(92)$ & $60.1(2)$ & $\mathrm{B}(83)-\mathrm{B}(89)-\mathrm{B}(92)$ & $108.3(3)$ \\
\hline$C(90)-B(90)-C(05)$ & $121.2(4)$ & $\mathrm{C}(90)-\mathrm{B}(90)-\mathrm{B}(89)$ & $124.3(3)$ \\
\hline $\mathrm{C}(05)-\mathrm{B}(90)-\mathrm{B}(89)$ & $108.1(4)$ & $\mathrm{C}(90)-\mathrm{B}(90)-\mathrm{B}(92)$ & $123.7(4)$ \\
\hline $\mathrm{C}(05)-\mathrm{B}(90)-\mathrm{B}(92)$ & $103.2(4)$ & $\mathrm{B}(89)-\mathrm{B}(90)-\mathrm{B}(92)$ & $60.4(2)$ \\
\hline $\mathrm{C}(90)-\mathrm{B}(90)-\mathrm{B}(84)$ & $121.0(4)$ & $\mathrm{C}(05)-\mathrm{B}(90)-\mathrm{B}(84)$ & $63.7(4)$ \\
\hline $\mathrm{B}(89)-\mathrm{B}(90)-\mathrm{B}(84)$ & $59.8(2)$ & $\mathrm{B}(92)-\mathrm{B}(90)-\mathrm{B}(84)$ & $108.3(3)$ \\
\hline $\mathrm{C}(90)-\mathrm{B}(90)-\mathrm{B}(91)$ & $120.5(3)$ & $\mathrm{C}(05)-\mathrm{B}(90)-\mathrm{B}(91)$ & $54.7(4)$ \\
\hline $\mathrm{B}(89)-\mathrm{B}(90)-\mathrm{B}(91)$ & $107.9(3)$ & $\mathrm{B}(92)-\mathrm{B}(90)-\mathrm{B}(91)$ & $59.6(2)$ \\
\hline $\mathrm{B}(84)-\mathrm{B}(90)-\mathrm{B}(91)$ & 108.1(3) & $\mathrm{C}(90)-\mathrm{B}(90)-\mathrm{B}(85)$ & $122.0(5)$ \\
\hline $\mathrm{C}(05)-\mathrm{B}(90)-\mathrm{B}(85)$ & $9.3(5)$ & $\mathrm{B}(89)-\mathrm{B}(90)-\mathrm{B}(85)$ & $102.7(4)$ \\
\hline $\mathrm{B}(92)-\mathrm{B}(90)-\mathrm{B}(85)$ & $107.5(4)$ & $\mathrm{B}(84)-\mathrm{B}(90)-\mathrm{B}(85)$ & $54.8(4)$ \\
\hline $\mathrm{B}(91)-\mathrm{B}(90)-\mathrm{B}(85)$ & $63.2(4)$ & $\mathrm{C}(05)-\mathrm{B}(91)-\mathrm{C}(91)$ & $120.1(5)$ \\
\hline $\mathrm{C}(05)-\mathrm{B}(91)-\mathrm{B}(86)$ & $63.4(5)$ & $\mathrm{C}(91)-\mathrm{B}(91)-\mathrm{B}(86)$ & $116.6(5)$ \\
\hline $\mathrm{C}(05)-\mathrm{B}(91)-\mathrm{B}(87)$ & $107.9(4)$ & $\mathrm{C}(91)-\mathrm{B}(91)-\mathrm{B}(87)$ & $123.2(4)$ \\
\hline $\mathrm{B}(86)-\mathrm{B}(91)-\mathrm{B}(87)$ & $60.1(4)$ & $\mathrm{C}(05)-\mathrm{B}(91)-\mathrm{B}(92)$ & 104.9(4) \\
\hline $\mathrm{C}(91)-\mathrm{B}(91)-\mathrm{B}(92)$ & $125.7(4)$ & $\mathrm{B}(86)-\mathrm{B}(91)-\mathrm{B}(92)$ & $109.6(5)$ \\
\hline $\mathrm{B}(87)-\mathrm{B}(91)-\mathrm{B}(92)$ & $60.4(2)$ & $\mathrm{C}(05)-\mathrm{B}(91)-\mathrm{B}(90)$ & $56.6(4)$ \\
\hline $\mathrm{C}(91)-\mathrm{B}(91)-\mathrm{B}(90)$ & $122.7(3)$ & $\mathrm{B}(86)-\mathrm{B}(91)-\mathrm{B}(90)$ & $109.9(5)$ \\
\hline $\mathrm{B}(87)-\mathrm{B}(91)-\mathrm{B}(90)$ & $108.0(3)$ & $\mathrm{B}(92)-\mathrm{B}(91)-\mathrm{B}(90)$ & $59.9(2)$ \\
\hline $\mathrm{C}(05)-\mathrm{B}(91)-\mathrm{C}(06)$ & $59.1(5)$ & $\mathrm{C}(91)-\mathrm{B}(91)-\mathrm{C}(06)$ & $123.8(5)$ \\
\hline $\mathrm{B}(86)-\mathrm{B}(91)-\mathrm{C}(06)$ & $7.5(6)$ & $\mathrm{B}(87)-\mathrm{B}(91)-\mathrm{C}(06)$ & $59.0(4)$ \\
\hline $\mathrm{B}(92)-\mathrm{B}(91)-\mathrm{C}(06)$ & 104.1(4) & $\mathrm{B}(90)-\mathrm{B}(91)-\mathrm{C}(06)$ & $102.8(4)$ \\
\hline $\mathrm{C}(05)-\mathrm{B}(91)-\mathrm{B}(85)$ & $5.4(5)$ & $\mathrm{C}(91)-\mathrm{B}(91)-\mathrm{B}(85)$ & $122.1(5)$ \\
\hline $\mathrm{B}(86)-\mathrm{B}(91)-\mathrm{B}(85)$ & $58.0(5)$ & $\mathrm{B}(87)-\mathrm{B}(91)-\mathrm{B}(85)$ & 103.4(4) \\
\hline $\mathrm{B}(92)-\mathrm{B}(91)-\mathrm{B}(85)$ & $105.6(4)$ & $\mathrm{B}(90)-\mathrm{B}(91)-\mathrm{B}(85)$ & $60.5(4)$ \\
\hline $\mathrm{C}(06)-\mathrm{B}(91)-\mathrm{B}(85)$ & $53.7(5)$ & $\mathrm{C}(92)-\mathrm{B}(92)-\mathrm{B}(91)$ & $121.3(4)$ \\
\hline $\mathrm{C}(92)-\mathrm{B}(92)-\mathrm{B}(90)$ & $121.3(3)$ & $\mathrm{B}(91)-\mathrm{B}(92)-\mathrm{B}(90)$ & $60.5(2)$ \\
\hline $\mathrm{C}(92)-\mathrm{B}(92)-\mathrm{B}(87)$ & $121.9(3)$ & $\mathrm{B}(91)-\mathrm{B}(92)-\mathrm{B}(87)$ & $59.8(2)$ \\
\hline $\mathrm{B}(90)-\mathrm{B}(92)-\mathrm{B}(87)$ & 108.1(3) & $\mathrm{C}(92)-\mathrm{B}(92)-\mathrm{B}(88)$ & $122.7(4)$ \\
\hline $\mathrm{B}(91)-\mathrm{B}(92)-\mathrm{B}(88)$ & $107.5(3)$ & $\mathrm{B}(90)-\mathrm{B}(92)-\mathrm{B}(88)$ & 107.4(3) \\
\hline $\mathrm{B}(87)-\mathrm{B}(92)-\mathrm{B}(88)$ & $59.8(2)$ & $\mathrm{C}(92)-\mathrm{B}(92)-\mathrm{B}(89)$ & $122.1(3)$ \\
\hline $\mathrm{B}(91)-\mathrm{B}(92)-\mathrm{B}(89)$ & $107.9(3)$ & $\mathrm{B}(90)-\mathrm{B}(92)-\mathrm{B}(89)$ & $59.5(2)$ \\
\hline $\mathrm{B}(87)-\mathrm{B}(92)-\mathrm{B}(89)$ & $107.8(3)$ & $\mathrm{B}(88)-\mathrm{B}(92)-\mathrm{B}(89)$ & $59.8(2)$ \\
\hline$C(3 A)-C(1 A)-C(2 A)$ & $106.8(9)$ & $C(3 A)-C(1 A)-P(1)$ & $117.2(6)$ \\
\hline$C(2 A)-C(1 A)-P(1)$ & $120.4(8)$ & $C(5 A)-C(4 A)-C(6 A)$ & $109.9(15)$ \\
\hline$C(5 A)-C(4 A)-P(1)$ & $106.6(9)$ & $C(6 A)-C(4 A)-P(1)$ & $109.8(12)$ \\
\hline$C(8 A)-C(7 A)-C(9 A)$ & $130(2)$ & $C(8 A)-C(7 A)-P(1)$ & $122.4(11)$ \\
\hline$C(9 A)-C(7 A)-P(1)$ & $91.9(13)$ & & \\
\hline
\end{tabular}

Symmetry transformations used to generate equivalent atoms: \#1-x+1--y+1--z+1---- 
Table S4. Crystal data and structure refinement for $\mathbf{1 b}$.

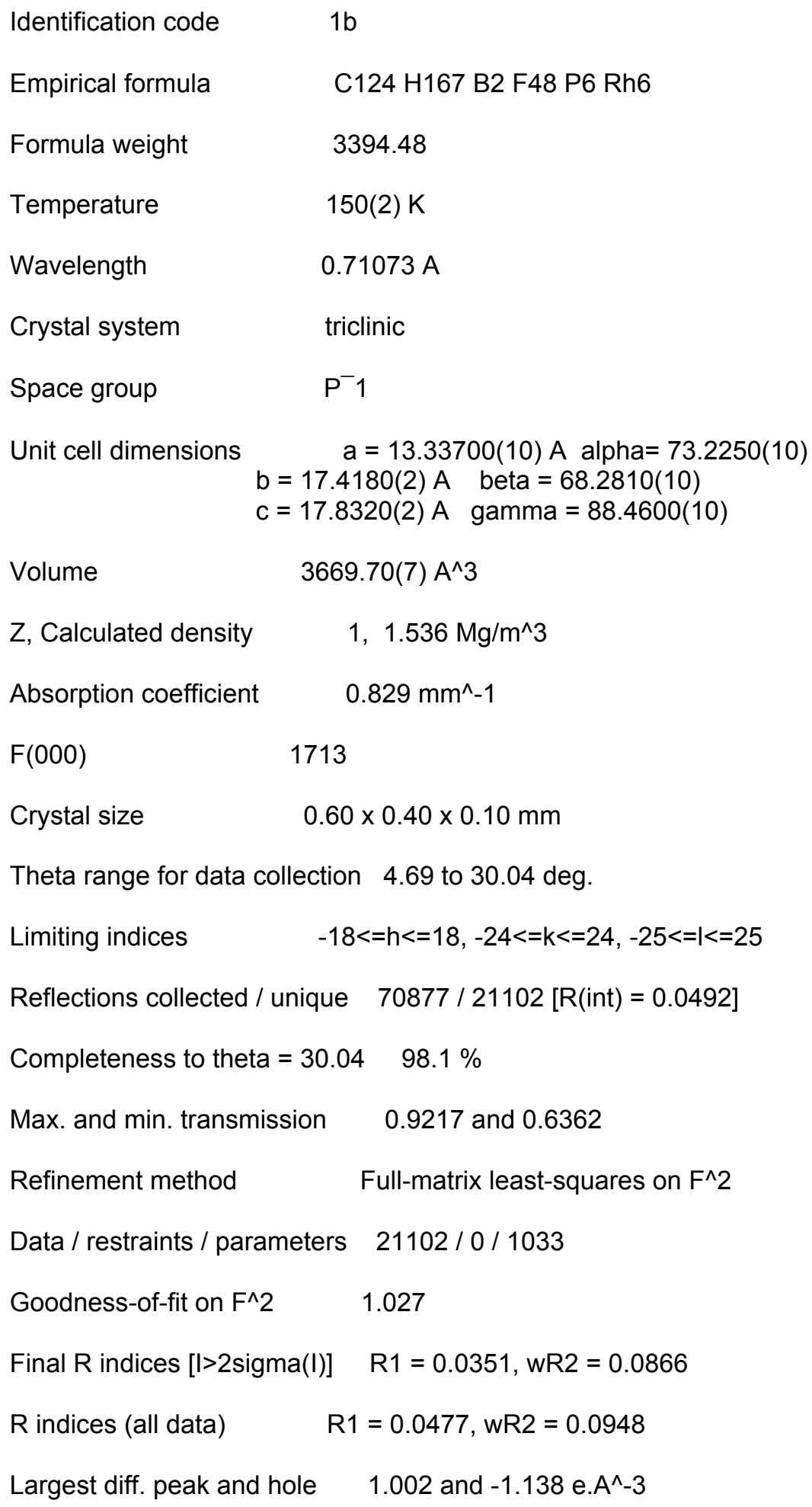


Table S5. Bond lengths $[\mathrm{A}]$ and angle $[\mathrm{deg}]$ for $1 \mathrm{~b}$.

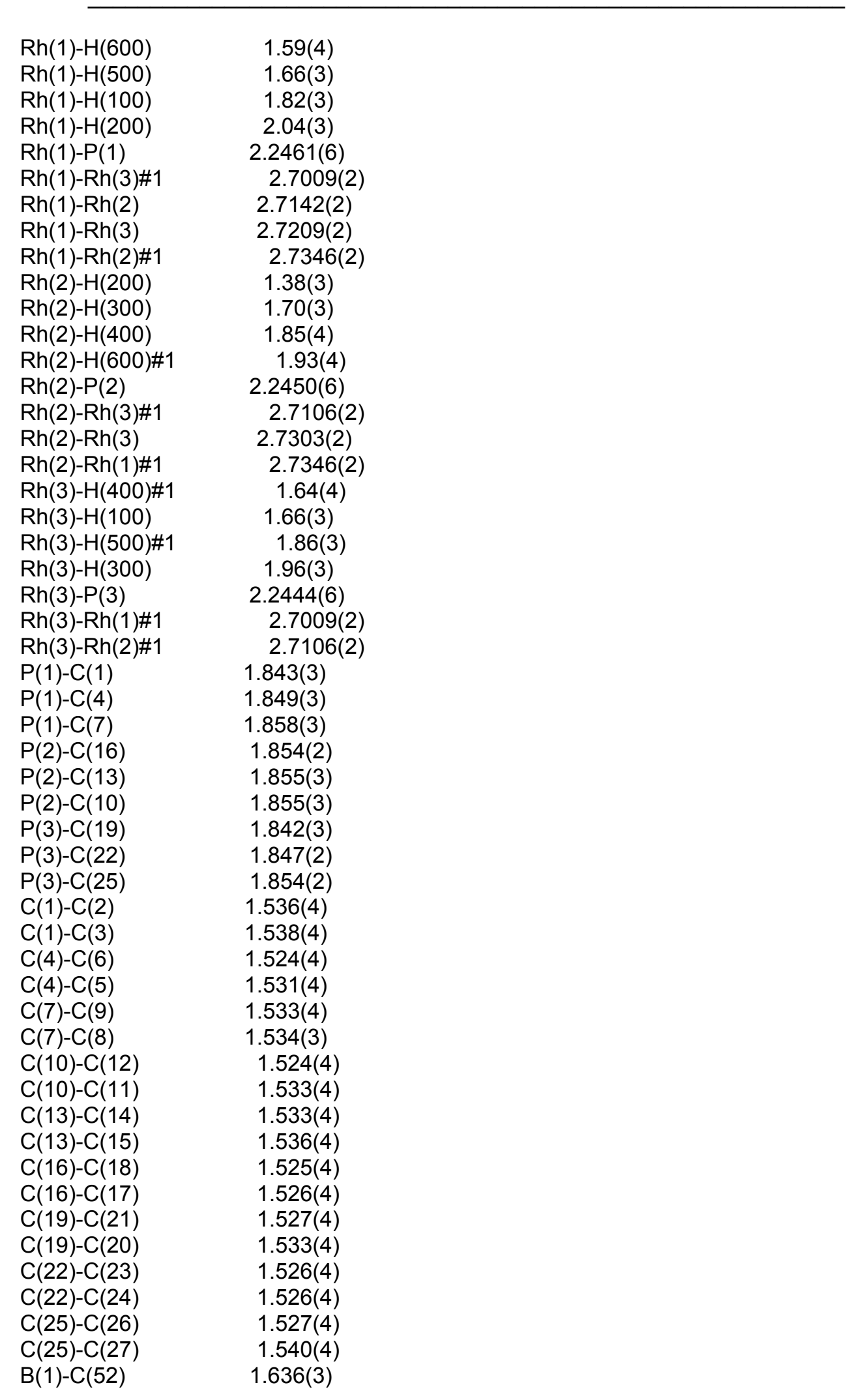




\begin{tabular}{|c|c|}
\hline$B(1)-C(36)$ & $1.640(4)$ \\
\hline$B(1)-C(44)$ & $1.643(4)$ \\
\hline$B(1)-C(28)$ & $1.645(3)$ \\
\hline$C(28)-C(33)$ & $1.395(3)$ \\
\hline$C(28)-C(29)$ & 1.404(3) \\
\hline$C(29)-C(30)$ & $1.381(3)$ \\
\hline$C(30)-C(31)$ & $1.386(3)$ \\
\hline$C(30)-C(34)$ & $1.499(3)$ \\
\hline$C(31)-C(32)$ & $1.388(3)$ \\
\hline$C(32)-C(33)$ & $1.391(3)$ \\
\hline$C(32)-C(35)$ & $1.501(3)$ \\
\hline$C(34)-F(3)$ & $1.313(4)$ \\
\hline$C(34)-F(1)$ & $1.320(3)$ \\
\hline$C(34)-F(2)$ & $1.338(3)$ \\
\hline$C(35)-F(4)$ & $1.322(3)$ \\
\hline$C(35)-F(5)$ & $1.333(3)$ \\
\hline$C(35)-F(6)$ & $1.343(3)$ \\
\hline$C(36)-C(37)$ & $1.394(3)$ \\
\hline$C(36)-C(41)$ & $1.408(4)$ \\
\hline$C(37)-C(38)$ & $1.394(4)$ \\
\hline$C(38)-C(39)$ & $1.378(4)$ \\
\hline$C(38)-C(42)$ & $1.510(4)$ \\
\hline$C(39)-C(40)$ & $1.392(4)$ \\
\hline$C(40)-C(41)$ & $1.385(4)$ \\
\hline$C(40)-C(43)$ & $1.504(4)$ \\
\hline$C(42)-F(7)$ & $1.173(12)$ \\
\hline$C(42)-F(8)$ & $1.286(11)$ \\
\hline$C(42)-F(9 A)$ & $1.289(12)$ \\
\hline$C(42)-F(9)$ & $1.324(9)$ \\
\hline$C(42)-F(8 A)$ & $1.352(10)$ \\
\hline$C(42)-F(7 A)$ & $1.444(10)$ \\
\hline$C(43)-F(12)$ & $1.308(5)$ \\
\hline$C(43)-F(10)$ & $1.320(4)$ \\
\hline$C(43)-F(11)$ & $1.331(6)$ \\
\hline $\mathrm{C}(44)-\mathrm{C}(45)$ & $1.401(3)$ \\
\hline$C(44)-C(49)$ & $1.402(3)$ \\
\hline$C(45)-C(46)$ & 1.392(3) \\
\hline$C(46)-C(47)$ & $1.384(4)$ \\
\hline$C(46)-C(50)$ & 1.495(4) \\
\hline$C(47)-C(48)$ & $1.383(4)$ \\
\hline $\mathrm{C}(48)-\mathrm{C}(49)$ & 1.392(3) \\
\hline$C(48)-C(51)$ & $1.500(4)$ \\
\hline$C(50)-F(14 A)$ & $1.259(8)$ \\
\hline$C(50)-F(13)$ & $1.282(11)$ \\
\hline$C(50)-F(15 A)$ & $1.290(12)$ \\
\hline$C(50)-F(15)$ & 1.335(11) \\
\hline$C(50)-F(13 A)$ & $1.350(13)$ \\
\hline$C(50)-F(14)$ & 1.396(9) \\
\hline$C(51)-F(17)$ & $1.274(13)$ \\
\hline$C(51)-F(18 A)$ & $1.281(18)$ \\
\hline$C(51)-F(16)$ & $1.301(9)$ \\
\hline$C(51)-F(17 A)$ & $1.338(14)$ \\
\hline$C(51)-F(18)$ & $1.36(2)$ \\
\hline$C(51)-F(16 A)$ & $1.387(9)$ \\
\hline$C(52)-C(57)$ & $1.397(4)$ \\
\hline$C(52)-C(53)$ & $1.398(3)$ \\
\hline
\end{tabular}




\begin{tabular}{|c|c|}
\hline$C(53)-C(54)$ & $1.392(4)$ \\
\hline$C(54)-C(55)$ & $1.382(4)$ \\
\hline$C(54)-C(58)$ & $1.498(5)$ \\
\hline$C(55)-C(56)$ & $1.368(4)$ \\
\hline$C(56)-C(57)$ & $1.392(4)$ \\
\hline$C(56)-C(59)$ & $1.514(5)$ \\
\hline$C(58)-F(19 A)$ & $1.16(2)$ \\
\hline$C(58)-F(21 A)$ & $1.231(8)$ \\
\hline$C(58)-F(20)$ & $1.292(15)$ \\
\hline$C(58)-F(20 A)$ & $1.327(18)$ \\
\hline$C(58)-F(19)$ & $1.360(18)$ \\
\hline$C(58)-F(21)$ & $1.392(10)$ \\
\hline$C(59)-F(23 A)$ & $1.222(15)$ \\
\hline$C(59)-F(22 A)$ & $1.256(15)$ \\
\hline$C(59)-F(24)$ & $1.257(9)$ \\
\hline $\mathrm{C}(59)-\mathrm{F}(22)$ & $1.33(2)$ \\
\hline$C(59)-F(23)$ & $1.357(16)$ \\
\hline$C(59)-F(24 A)$ & $1.378(14)$ \\
\hline$C(60)-C(61)$ & $1.254(11)$ \\
\hline$C(60)-F(30)$ & $1.337(16)$ \\
\hline$C(60)-C(62) \# 2$ & $1.467(13)$ \\
\hline$F(30 A)-C(61)$ & $1.39(2)$ \\
\hline$C(61)-C(62)$ & $1.331(15)$ \\
\hline $\mathrm{C}(62)-\mathrm{C}(60) \# 2$ & $1.467(13)$ \\
\hline$H(600)-R h(1)-H(500)$ & $88.1(18)$ \\
\hline$H(600)-R h(1)-H(100)$ & $90.6(17)$ \\
\hline$H(500)-R h(1)-H(100)$ & 169.1(16) \\
\hline$H(600)-R h(1)-H(200)$ & $160.5(16)$ \\
\hline$H(500)-R h(1)-H(200)$ & $82.0(14)$ \\
\hline$H(100)-R h(1)-H(200)$ & $96.0(14)$ \\
\hline $\mathrm{H}(600)-\mathrm{Rh}(1)-\mathrm{P}(1)$ & $89.4(13)$ \\
\hline$H(500)-R h(1)-P(1)$ & $89.9(11)$ \\
\hline$H(100)-R h(1)-P(1)$ & $100.9(11)$ \\
\hline$H(200)-R h(1)-P(1)$ & 107.3(8) \\
\hline $\mathrm{H}(600)-\mathrm{Rh}(1)-\mathrm{Rh}(3) \# 1$ & $88.5(13)$ \\
\hline $\mathrm{H}(500)-\mathrm{Rh}(1)-\mathrm{Rh}(3) \# 1$ & $42.7(11)$ \\
\hline $\mathrm{H}(100)-\mathrm{Rh}(1)-\mathrm{Rh}(3) \# 1$ & $126.4(11)$ \\
\hline $\mathrm{H}(200)-\mathrm{Rh}(1)-\mathrm{Rh}(3) \# 1$ & $72.8(8)$ \\
\hline$P(1)-R h(1)-R h(3) \# 1$ & $132.657(17)$ \\
\hline$H(600)-R h(1)-R h(2)$ & $134.1(13)$ \\
\hline$H(500)-R h(1)-R h(2)$ & $88.9(12)$ \\
\hline$H(100)-R h(1)-R h(2)$ & $84.2(11)$ \\
\hline$H(200)-\operatorname{Rh}(1)-\operatorname{Rh}(2)$ & $29.6(8)$ \\
\hline$P(1)-R h(1)-R h(2)$ & $136.395(17)$ \\
\hline $\mathrm{Rh}(3) \# 1-\mathrm{Rh}(1)-\mathrm{Rh}(2)$ & $60.074(6)$ \\
\hline$H(600)-R h(1)-R h(3)$ & $89.7(14)$ \\
\hline $\mathrm{H}(500)-\mathrm{Rh}(1)-\mathrm{Rh}(3)$ & $132.6(11)$ \\
\hline$H(100)-R h(1)-R h(3)$ & $36.6(11)$ \\
\hline $\mathrm{H}(200)-\mathrm{Rh}(1)-\mathrm{Rh}(3)$ & $84.8(8)$ \\
\hline$P(1)-R h(1)-R h(3)$ & $137.449(17)$ \\
\hline $\mathrm{Rh}(3) \# 1-\mathrm{Rh}(1)-\mathrm{Rh}(3)$ & $89.832(7)$ \\
\hline
\end{tabular}




$\begin{array}{lc} & \\ \text { Rh(2)-Rh(1)-Rh(3) } & 60.309(6) \\ \text { H(600)-Rh(1)-Rh(2)\#1 } & 43.8(13) \\ \text { H(500)-Rh(1)-Rh(2)\#1 } & 88.1(12) \\ \text { H(100)-Rh(1)-Rh(2)\#1 } & 83.5(11) \\ \text { H(200)-Rh(1)-Rh(2)\#1 } & 118.7(8) \\ \text { P(1)-Rh(1)-Rh(2)\#1 } & 133.121(17) \\ \text { Rh(3)\#1-Rh(1)-Rh(2)\#1 } & 60.301(6) \\ \text { Rh(2)-Rh(1)-Rh(2)\#1 } & 90.399(7) \\ \text { Rh(3)-Rh(1)-Rh(2)\#1 } & 59.585(6) \\ \text { H(200)-Rh(2)-H(300) } & 102.3(17) \\ \text { H(200)-Rh(2)-H(400) } & 76.8(17) \\ \text { H(300)-Rh(2)-H(400) } & 171.2(17) \\ \text { H(200)-Rh(2)-H(600)\#1 } & 165.5(17) \\ \text { H(300)-Rh(2)-H(600)\#1 } & 88.5(16) \\ \text { H(400)-Rh(2)-H(600)\#1 } & 91.0(16) \\ \text { H(200)-Rh(2)-P(2) } & 85.9(13) \\ \text { H(300)-Rh(2)-P(2) } & 88.7(12) \\ \text { H(400)-Rh(2)-P(2) } & 99.9(12) \\ \text { H(600)\#1-Rh(2)-P(2) } & 104.3(11) \\ \text { H(200)-Rh(2)-Rh(3)\#1 } & 82.0(12) \\ \text { H(300)-Rh(2)-Rh(3)\#1 } & 134.9(12) \\ \text { H(400)-Rh(2)-Rh(3)\#1 } & 36.4(12) \\ \text { H(600)\#1-Rh(2)-Rh(3)\#1 } & 83.5(11) \\ \text { P(2)-Rh(2)-Rh(3)\#1 } & 136.304(17) \\ \text { H(200)-Rh(2)-Rh(1) } & 47.1(13) \\ \text { H(300)-Rh(2)-Rh(1) } & 90.2(11) \\ \text { H(400)-Rh(2)-Rh(1) } & 82.7(12) \\ \text { H(600)\#1-Rh(2)-Rh(1) } & 124.3(11) \\ \text { P(2)-Rh(2)-Rh(1) } & 131.388(17) \\ \text { Rh(3)\#1-Rh(2)-Rh(1) } & 59.720(6) \\ \text { H(200)-Rh(2)-Rh(3) } & 99.2(13) \\ \text { H(300)-Rh(2)-Rh(3) } & 45.4(12) \\ \text { H(400)-Rh(2)-Rh(3) } & 125.8(12) \\ \text { H(600)\#1-Rh(2)-Rh(3) } & 81.3(11) \\ \text { P(2)-Rh(2)-Rh(3) } & 134.036(17) \\ \text { Rh(3)\#1-Rh(2)-Rh(3) } & 89.433(7) \\ \text { Rh(1)-Rh(2)-Rh(3) } & 59.967(6) \\ \text { H(200)-Rh(2)-Rh(1)\#1 } & 134.3(13) \\ \text { H(300)-Rh(2)-Rh(1)\#1 } & 89.7(12) \\ \text { H(400)-Rh(2)-Rh(1)\#1 } & 85.0(12) \\ \text { H(600)\#1-Rh(2)-Rh(1)\#1 } & 34.7(11) \\ \mathrm{P}(2)-R h(2)-R h(1) \# 1 & 138.978(17) \\ \text { Rh(3)\#1-Rh(2)-Rh(1)\#1 } & 59.957(6) \\ \text { Rh(1)-Rh(2)-Rh(1)\#1 } & 89.601(7) \\ \text { Rh(3)-Rh(2)-Rh(1)\#1 } & 59.239(6) \\ \text { H(400)\#1-Rh(3)-H(100) } & 91.5(18) \\ \text { H(400)\#1-Rh(3)-H(500)\#1 } & 88.8(17) \\ \text { H(100)-Rh(3)-H(500)\#1 } & 168.2(16) \\ \text { H(400)\#1-Rh(3)-H(300) } & 170.9(17) \\ \text { H(100)-Rh(3)-H(300) } & 89.2(16) \\ \text { H(500)\#1-Rh(3)-H(300) } & 88.7(14) \\ \text { H(400)\#1-Rh(3)-P(3) } & 89.9(13) \\ \text { H(100)-Rh(3)-P(3) } & 92.1(12) \\ \text { H(500)\#1-Rh(3)-P(3) } & 99.7(10) \\ \text { H(300)-Rh(3)-P(3) } & 99.2(10) \\ \text { H(400)\#1-Rh(3)-Rh(1)\#1 } & 87.0(13) \\ & \end{array}$




$\begin{array}{lc}\text { H(100)-Rh(3)-Rh(1)\#1 } & 130.9(12) \\ \mathrm{H}(500) \# 1-\mathrm{Rh}(3)-\mathrm{Rh}(1) \# 1 & 37.3(10) \\ \mathrm{H}(300)-\mathrm{Rh}(3)-\mathrm{Rh}(1) \# 1 & 85.7(10) \\ \mathrm{P}(3)-\mathrm{Rh}(3)-\mathrm{Rh}(1) \# 1 & 136.923(17) \\ \mathrm{H}(400) \# 1-\mathrm{Rh}(3)-\mathrm{Rh}(2) \# 1 & 42.1(13) \\ \mathrm{H}(100)-\mathrm{Rh}(3)-\mathrm{Rh}(2) \# 1 & 87.2(12) \\ \mathrm{H}(500) \# 1-\mathrm{Rh}(3)-\mathrm{Rh}(2) \# 1 & 85.1(10) \\ \mathrm{H}(300)-\mathrm{Rh}(3)-\mathrm{Rh}(2) \# 1 & 128.9(10) \\ \mathrm{P}(3)-\mathrm{Rh}(3)-\mathrm{Rh}(2) \# 1 & 131.845(17) \\ \mathrm{Rh}(1) \# 1-\mathrm{Rh}(3)-\mathrm{Rh}(2) \# 1 & 60.206(6) \\ \mathrm{H}(400) \# 1-\mathrm{Rh}(3)-\mathrm{Rh}(1) & 89.5(13) \\ \mathrm{H}(100)-\mathrm{Rh}(3)-\mathrm{Rh}(1) & 40.7(12) \\ \mathrm{H}(500) \# 1-\mathrm{Rh}(3)-\mathrm{Rh}(1) & 127.5(10) \\ \mathrm{H}(300)-\mathrm{Rh}(3)-\mathrm{Rh}(1) & 85.0(10) \\ \mathrm{P}(3)-\mathrm{Rh}(3)-\mathrm{Rh}(1) & 132.788(17) \\ \mathrm{Rh}(1) \# 1-\mathrm{Rh}(3)-\mathrm{Rh}(1) & 90.168(7) \\ \mathrm{Rh}(2) \# 1-\mathrm{Rh}(3)-\mathrm{Rh}(1) & 60.458(6) \\ \mathrm{H}(400) \# 1-\mathrm{Rh}(3)-\mathrm{Rh}(2) & 132.6(13) \\ \mathrm{H}(100)-\mathrm{Rh}(3)-\mathrm{Rh}(2) & 86.6(12) \\ \mathrm{H}(500) \# 1-\mathrm{Rh}(3)-\mathrm{Rh}(2) & 84.5(10) \\ \mathrm{H}(300)-\mathrm{Rh}(3)-\mathrm{Rh}(2) & 38.4(10) \\ \mathrm{P}(3)-\mathrm{Rh}(3)-\mathrm{Rh}(2) & 137.497(17) \\ \mathrm{Rh}(1) \# 1-\mathrm{Rh}(3)-\mathrm{Rh}(2) & 60.460(6) \\ \mathrm{Rh}(2) \# 1-\mathrm{Rh}(3)-\mathrm{Rh}(2) & 90.567(7) \\ \mathrm{Rh}(1)-\mathrm{Rh}(3)-\mathrm{Rh}(2) & 59.724(6) \\ \mathrm{C}(1)-\mathrm{P}(1)-\mathrm{C}(4) & 104.34(13) \\ \mathrm{C}(1)-\mathrm{P}(1)-\mathrm{C}(7) & 104.29(12) \\ \mathrm{C}(4)-\mathrm{P}(1)-\mathrm{C}(7) & 110.64(12) \\ \mathrm{C}(1)-\mathrm{P}(1)-\mathrm{Rh}(1) & 112.93(8) \\ \mathrm{C}(4)-\mathrm{P}(1)-\mathrm{Rh}(1) & 113.63(8) \\ \mathrm{C}(7)-\mathrm{P}(1)-\mathrm{Rh}(1) & 110.49(8) \\ \mathrm{C}(16)-\mathrm{P}(2)-\mathrm{C}(13) & 103.40(12) \\ \mathrm{C}(16)-\mathrm{P}(2)-\mathrm{C}(10) & 104.56(12) \\ \mathrm{C}(13)-\mathrm{P}(2)-\mathrm{C}(10) & 111.31(13) \\ \mathrm{C}(16)-\mathrm{P}(2)-\mathrm{Rh}(2) & 111.10(8) \\ \mathrm{C}(13)-\mathrm{P}(2)-\mathrm{Rh}(2) & 113.15(8) \\ \mathrm{C}(10)-\mathrm{P}(2)-\mathrm{Rh}(2) & 112.59(9) \\ \mathrm{C}(19)-\mathrm{P}(3)-\mathrm{C}(22) & 104.43(12) \\ \mathrm{C}(19)-\mathrm{P}(3)-\mathrm{C}(25) & 103.41(12) \\ \mathrm{C}(22)-\mathrm{P}(3)-\mathrm{C}(25) & 111.71(12) \\ \mathrm{C}(19)-\mathrm{P}(3)-\mathrm{Rh}(3) & 112.60(8) \\ \mathrm{C}(22)-\mathrm{P}(3)-\mathrm{Rh}(3) & 111.60(8) \\ \mathrm{C}(25)-\mathrm{P}(3)-\mathrm{Rh}(3) & 112.54(8) \\ \mathrm{C}(2)-\mathrm{C}(1)-\mathrm{C}(3) & 110.9(2) \\ \mathrm{C}(2)-\mathrm{C}(1)-\mathrm{P}(1) & 111.11(18) \\ \mathrm{C}(3)-\mathrm{C}(1)-\mathrm{P}(1) & 111.80(19) \\ \mathrm{C}(6)-\mathrm{C}(4)-\mathrm{C}(5) & 109.9(2) \\ \mathrm{C}(6)-\mathrm{C}(4)-\mathrm{P}(1) & 114.09(19) \\ \mathrm{C}(5)-\mathrm{C}(4)-\mathrm{P}(1) & 114.6(2) \\ \mathrm{C}(9)-\mathrm{C}(7)-\mathrm{C}(8) & 109.9(2) \\ \mathrm{C}(9)-\mathrm{C}(7)-\mathrm{P}(1) & 112.93(18) \\ \mathrm{C}(8)-\mathrm{C}(7)-\mathrm{P}(1) & 116.3(2) \\ \mathrm{C}(12)-\mathrm{C}(10)-\mathrm{C}(11) & 109.8(3) \\ \mathrm{C}(12)-\mathrm{C}(10)-\mathrm{P}(2) & 115.6(2) \\ \mathrm{C}(11)-\mathrm{C}(10)-\mathrm{P}(2) & 114.38(18) \\ \mathrm{C}(14)-\mathrm{C}(13)-\mathrm{C}(15) & 110.3(2) \\ & \end{array}$




$\begin{array}{lc}\mathrm{C}(14)-\mathrm{C}(13)-\mathrm{P}(2) & 113.0(2) \\ \mathrm{C}(15)-\mathrm{C}(13)-\mathrm{P}(2) & 116.4(2) \\ \mathrm{C}(18)-\mathrm{C}(16)-\mathrm{C}(17) & 110.7(2) \\ \mathrm{C}(18)-\mathrm{C}(16)-\mathrm{P}(2) & 111.96(19) \\ \mathrm{C}(17)-\mathrm{C}(16)-\mathrm{P}(2) & 110.96(17) \\ \mathrm{C}(21)-\mathrm{C}(19)-\mathrm{C}(20) & 110.5(3) \\ \mathrm{C}(21)-\mathrm{C}(19)-\mathrm{P}(3) & 111.41(18) \\ \mathrm{C}(20)-\mathrm{C}(19)-\mathrm{P}(3) & 112.17(18) \\ \mathrm{C}(23)-\mathrm{C}(22)-\mathrm{C}(24) & 109.4(3) \\ \mathrm{C}(23)-\mathrm{C}(22)-\mathrm{P}(3) & 114.07(19) \\ \mathrm{C}(24)-\mathrm{C}(22)-\mathrm{P}(3) & 115.75(19) \\ \mathrm{C}(26)-\mathrm{C}(25)-\mathrm{C}(27) & 110.8(2) \\ \mathrm{C}(26)-\mathrm{C}(25)-\mathrm{P}(3) & 113.06(18) \\ \mathrm{C}(27)-\mathrm{C}(25)-\mathrm{P}(3) & 115.77(19) \\ \mathrm{C}(52)-\mathrm{B}(1)-\mathrm{C}(36) & 105.05(19) \\ \mathrm{C}(52)-\mathrm{B}(1)-\mathrm{C}(44) & 112.87(19) \\ \mathrm{C}(36)-\mathrm{B}(1)-\mathrm{C}(44) & 113.70(19) \\ \mathrm{C}(52)-\mathrm{B}(1)-\mathrm{C}(28) & 112.97(19) \\ \mathrm{C}(36)-\mathrm{B}(1)-\mathrm{C}(28) & 109.38(19) \\ \mathrm{C}(44)-\mathrm{B}(1)-\mathrm{C}(28) & 103.05(18) \\ \mathrm{C}(33)-\mathrm{C}(28)-\mathrm{C}(29) & 115.7(2) \\ \mathrm{C}(33)-\mathrm{C}(28)-\mathrm{B}(1) & 124.3(2) \\ \mathrm{C}(29)-\mathrm{C}(28)-\mathrm{B}(1) & 119.9(2) \\ \mathrm{C}(30)-\mathrm{C}(29)-\mathrm{C}(28) & 122.4(2) \\ \mathrm{C}(29)-\mathrm{C}(30)-\mathrm{C}(31) & 121.2(2) \\ \mathrm{C}(29)-\mathrm{C}(30)-\mathrm{C}(34) & 118.4(2) \\ \mathrm{C}(31)-\mathrm{C}(30)-\mathrm{C}(34) & 120.4(2) \\ \mathrm{C}(30)-\mathrm{C}(31)-\mathrm{C}(32) & 117.4(2) \\ \mathrm{C}(31)-\mathrm{C}(32)-\mathrm{C}(33) & 121.3(2) \\ \mathrm{C}(31)-\mathrm{C}(32)-\mathrm{C}(35) & 120.3(2) \\ \mathrm{C}(33)-\mathrm{C}(32)-\mathrm{C}(35) & 118.4(2) \\ \mathrm{C}(32)-\mathrm{C}(33)-\mathrm{C}(28) & 121.9(2) \\ \mathrm{F}(3)-\mathrm{C}(34)-\mathrm{F}(1) & 106.7(3) \\ \mathrm{F}(3)-\mathrm{C}(34)-\mathrm{F}(2) & 106.3(3) \\ \mathrm{F}(1)-\mathrm{C}(34)-\mathrm{F}(2) & 106.3(2) \\ \mathrm{F}(3)-\mathrm{C}(34)-\mathrm{C}(30) & 112.4(2) \\ \mathrm{F}(1)-\mathrm{C}(34)-\mathrm{C}(30) & 113.1(2) \\ \mathrm{F}(2)-\mathrm{C}(34)-\mathrm{C}(30) & 111.6(2) \\ \mathrm{F}(4)-\mathrm{C}(35)-\mathrm{F}(5) & 106.8(2) \\ \mathrm{F}(4)-\mathrm{C}(35)-\mathrm{F}(6) & 107.5(2) \\ \mathrm{F}(5)-\mathrm{C}(35)-\mathrm{F}(6) & 104.1(2) \\ \mathrm{F}(4)-\mathrm{C}(35)-\mathrm{C}(32) & 113.5(2) \\ \mathrm{F}(5)-\mathrm{C}(35)-\mathrm{C}(32) & 112.6(2) \\ \mathrm{F}(6)-\mathrm{C}(35)-\mathrm{C}(32) & 111.8(2) \\ \mathrm{C}(37)-\mathrm{C}(36)-\mathrm{C}(41) & 115.9(2) \\ \mathrm{C}(37)-\mathrm{C}(36)-\mathrm{B}(1) & 124.1(2) \\ \mathrm{C}(41)-\mathrm{C}(36)-\mathrm{B}(1) & 119.9(2) \\ \mathrm{C}(36)-\mathrm{C}(37)-\mathrm{C}(38) & 122.1(2) \\ \mathrm{C}(39)-\mathrm{C}(38)-\mathrm{C}(37) & 121.2(2) \\ \mathrm{C}(39)-\mathrm{C}(38)-\mathrm{C}(42) & 119.7(3) \\ \mathrm{C}(37)-\mathrm{C}(38)-\mathrm{C}(42) & 119.1(3) \\ \mathrm{C}(38)-\mathrm{C}(39)-\mathrm{C}(40) & 117.9(3) \\ \mathrm{C}(41)-\mathrm{C}(40)-\mathrm{C}(40)-\mathrm{C}(43) & 121.0(3) \\ \mathrm{C}(39)-\mathrm{C}(40)-\mathrm{C}(43) & 118.9(3) \\ \mathrm{C}(40)-\mathrm{C}(41)-\mathrm{C}(36) & 120.0(3) \\ & 122.0(2) \\ & \end{array}$




\begin{tabular}{|c|c|}
\hline$(7)-C(42)-F(8)$ & \\
\hline$=(7)-C(42)-F(9 A)$ & $91.7(11)$ \\
\hline$F(8)-C(42)-F(9 A)$ & 118.2(9) \\
\hline$F(7)-C(42)-F(9)$ & $110.7(9)$ \\
\hline$F(8)-C(42)-F(9)$ & 104.6(8) \\
\hline$F(9 A)-C(42)-F(9)$ & \\
\hline$F(7)-C(42)-F(8 A)$ & 109. \\
\hline$(8)-C(42)-F(8 A)$ & $7.9(10)$ \\
\hline$(9 A)-C(42)-F(8 A)$ & $111.4(9)$ \\
\hline$=(9)-C(42)-F(8 A)$ & $96.9(8)$ \\
\hline$F(7)-C(42)-F(7 A)$ & $9.5(10)$ \\
\hline$F(8)-C(42)-F(7 A)$ & $97.9(6)$ \\
\hline$F(9 A)-C(42)-F(7 A)$ & \\
\hline$F(9)-C(42)-F(7 A)$ & \\
\hline$F(8 A)-C(42)-F(7 A)$ & \\
\hline$F(7)-C(42)-C(38)$ & 113 \\
\hline$F(8)-C(42)-C(38)$ & $112.4(6)$ \\
\hline$F(9 A)-C(42)-C(38)$ & $114.4(6)$ \\
\hline $\mathrm{F}(9)-\mathrm{C}(42)-\mathrm{C}(38)$ & $110.9(5)$ \\
\hline$F(8 A)-C(42)-C(38)$ & \\
\hline$F(7 A)-C(42)-C(3$ & \\
\hline 3$)-F(1$ & \\
\hline$F(12)-C($ & \\
\hline$F(10)-C(43)-F(1$ & 10 \\
\hline$F(12)-C(43)-C(40)$ & $.5(3)$ \\
\hline$F(10)-C(43)-C(40)$ & $113.5(3)$ \\
\hline$F(11)-C(43)-C(40)$ & $.1(3)$ \\
\hline (44)-C(49) & $3(2)$ \\
\hline$-B(1)$ & \\
\hline$-B(1)$ & \\
\hline $\mathrm{C}(46$ & (2) \\
\hline $\mathrm{C}(47)-\mathrm{C}(46)-\mathrm{C}(4$ & $.9(2)$ \\
\hline$C(47)-C(46)-C(50)$ & $.1(2)$ \\
\hline$C(45)-C(46)-C(50)$ & $9(2)$ \\
\hline$C(48)-C(47)-C(46)$ & $8(2)$ \\
\hline $\mathrm{C}(47)-\mathrm{C}(4$ & 1(2) \\
\hline$C(47)$ & \\
\hline $\mathrm{C}(4$ & \\
\hline $\mathrm{C}(48)-\mathrm{C}(4$ & 12 \\
\hline$F(14 A)-C(50)-F($ & (9) \\
\hline$F(14 A)-C(50)-F($ & $1.7(\varepsilon$ \\
\hline$F(13)-C(50)-F(1$ & $.5(8)$ \\
\hline D)-F(15) & $.0(7)$ \\
\hline & 2(8) \\
\hline & $5.2(10$ \\
\hline$F(1$ & 97 \\
\hline$-C(50)-F(13$ & $15.4(14$ \\
\hline$F(15 A)-C(50)-F($ & 1067 \\
\hline$F(15)-C(50)-F(13$ & $4(8)$ \\
\hline$F(14 A)-C(50)-F(14)$ & $0.4(6)$ \\
\hline$C(50)-F(14)$ & $111.2(10$ \\
\hline A) $-C(50)-F(14)$ & (9) \\
\hline$C(50)-F(14)$ & 104.9(8) \\
\hline (50) $ᄃ 4$ & $125.9(9)$ \\
\hline$F(14 A)-C(50)-C($ & $1150 / 1$ \\
\hline$F(13) \Gamma(50)-(16)$ & $114.3(6$ \\
\hline
\end{tabular}




\begin{tabular}{|c|c|}
\hline$F(15 A)-C(50)-C(46)$ & $114.3(6)$ \\
\hline$F(15)-C(50)-C(46)$ & 113.6(6) \\
\hline$F(13 A)-C(50)-C(46)$ & $108.9(7)$ \\
\hline$F(14)-C(50)-C(46)$ & $109.1(4)$ \\
\hline$F(17)-C(51)-F(18 A)$ & $114.9(9)$ \\
\hline$F(17)-C(51)-F(16)$ & $112.2(9)$ \\
\hline$F(18 A)-C(51)-F(16)$ & $80.6(10)$ \\
\hline$F(17)-C(51)-F(17 A)$ & $11.7(13)$ \\
\hline$F(18 A)-C(51)-F(17 A)$ & $108.6(10)$ \\
\hline$F(16)-C(51)-F(17 A)$ & $122.0(8)$ \\
\hline$F(17)-C(51)-F(18)$ & 102.1(10) \\
\hline$F(18 A)-C(51)-F(18)$ & $21.8(15)$ \\
\hline$F(16)-C(51)-F(18)$ & $102.0(8)$ \\
\hline$F(17 A)-C(51)-F(18)$ & $93.2(10)$ \\
\hline$F(17)-C(51)-F(16 A)$ & $88.7(8)$ \\
\hline$F(18 A)-C(51)-F(16 A)$ & 109.5(9) \\
\hline$F(16)-C(51)-F(16 A)$ & $31.3(5)$ \\
\hline$F(17 A)-C(51)-F(16 A)$ & $100.2(8)$ \\
\hline$F(18)-C(51)-F(16 A)$ & $129.0(8)$ \\
\hline$F(17)-C(51)-C(48)$ & $115.0(6)$ \\
\hline$F(18 A)-C(51)-C(48)$ & $115.2(7)$ \\
\hline$F(16)-C(51)-C(48)$ & $114.5(5)$ \\
\hline$F(17 A)-C(51)-C(48)$ & $112.0(6)$ \\
\hline$F(18)-C(51)-C(48)$ & $109.4(8)$ \\
\hline$F(16 A)-C(51)-C(48)$ & $110.3(4)$ \\
\hline$C(57)-C(52)-C(53)$ & $114.8(2)$ \\
\hline$C(57)-C(52)-B(1)$ & $122.3(2)$ \\
\hline$C(53)-C(52)-B(1)$ & $122.5(2)$ \\
\hline$C(54)-C(53)-C(52)$ & $122.5(2)$ \\
\hline$C(55)-C(54)-C(53)$ & $121.0(3)$ \\
\hline$C(55)-C(54)-C(58)$ & $119.1(3)$ \\
\hline C(53)-C(54)-C(58) & $120.0(3)$ \\
\hline$C(56)-C(55)-C(54)$ & $117.9(3)$ \\
\hline$C(55)-C(56)-C(57)$ & 121.1(3) \\
\hline$C(55)-C(56)-C(59)$ & 119.7(3) \\
\hline$C(57)-C(56)-C(59)$ & 119.1(3) \\
\hline$C(56)-C(57)-C(52)$ & $122.7(3)$ \\
\hline$F(19 A)-C(58)-F(21 A)$ & $115.2(12)$ \\
\hline$F(19 A)-C(58)-F(20)$ & $115.2(13)$ \\
\hline$F(21 A)-C(58)-F(20)$ & $74.1(12)$ \\
\hline$F(19 A)-C(58)-F(20 A)$ & $101.3(13)$ \\
\hline$F(21 A)-C(58)-F(20 A)$ & $97.5(13)$ \\
\hline$F(20)-C(58)-F(20 A)$ & $23.8(18)$ \\
\hline$F(19 A)-C(58)-F(19)$ & $12.7(16)$ \\
\hline$F(21 A)-C(58)-F(19)$ & $123.5(9)$ \\
\hline$F(20)-C(58)-F(19)$ & $106.7(12)$ \\
\hline$F(20 A)-C(58)-F(19)$ & $90.3(12)$ \\
\hline$F(19 A)-C(58)-F(21)$ & $86.3(12)$ \\
\hline$F(21 A)-C(58)-F(21)$ & $41.2(9)$ \\
\hline$F(20)-C(58)-F(21)$ & 113.1(13) \\
\hline$F(20 A)-C(58)-F(21)$ & $134.0(11)$ \\
\hline$F(19)-C(58)-F(21)$ & $98.5(10)$ \\
\hline$F(19 A)-C(58)-C(54)$ & $115.9(10)$ \\
\hline$F(21 A)-C(58)-C(54)$ & $116.0(5)$ \\
\hline$F(20)-C(58)-C(54)$ & 113.8(8) \\
\hline$F(20 A)-C(58)-C(54)$ & $107.5(10)$ \\
\hline
\end{tabular}




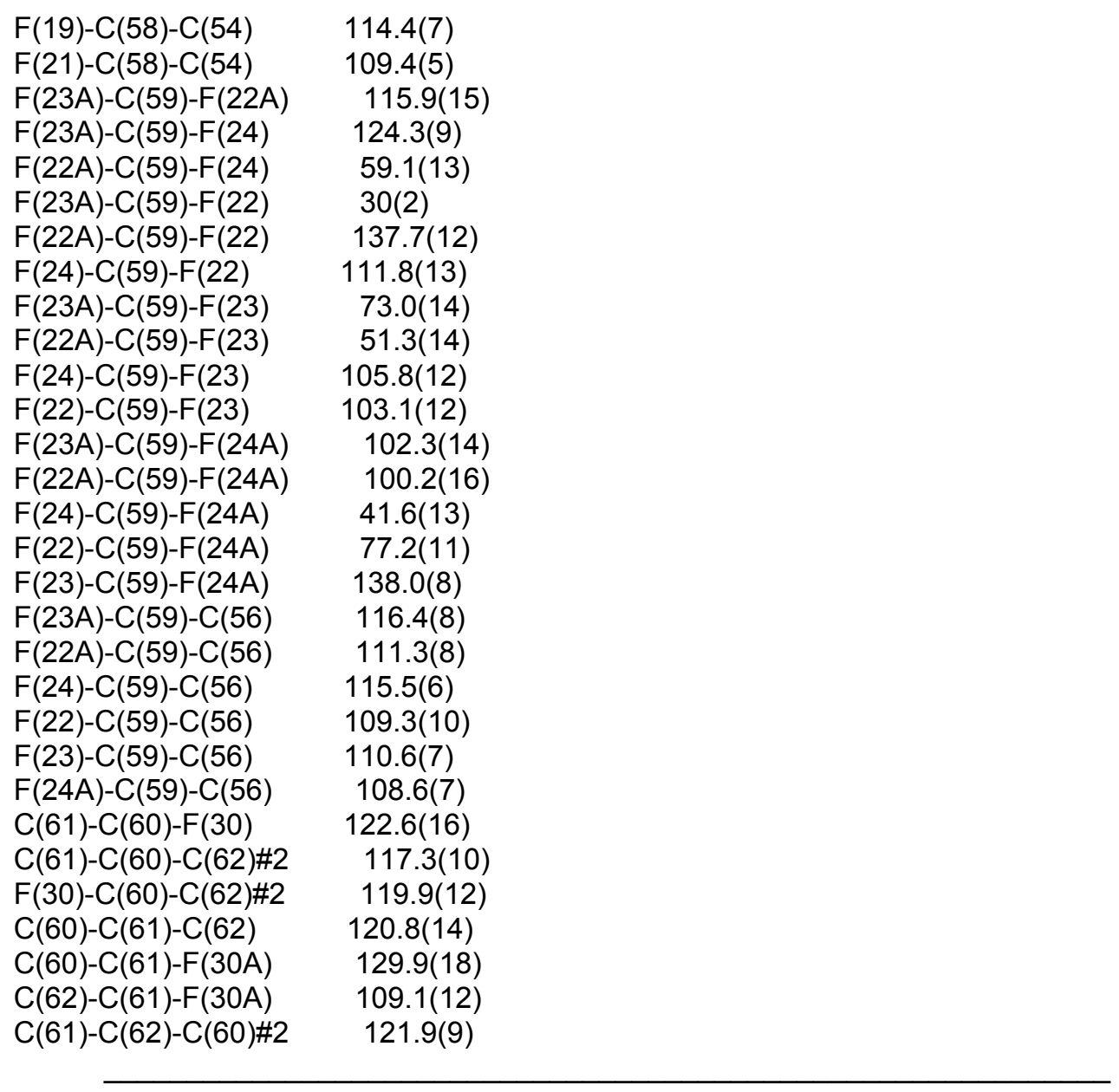

Symmetry transformations used to generate equivalent atoms:

$\# 1-x,-y,-z+2 \quad \# 2-x+1,-y+1,-z+1$ 


\section{Analysis of hydride positions in $1 \mathrm{a}$ and $1 \mathrm{~b}$ using HYDEX:}

Using HYDEX ${ }^{(5)}$ the best fit for the 12 hydrides confirmed from ${ }^{1} \mathrm{H}$ NMR experiments in $\mathbf{1 a}$ and $\mathbf{1 b}$ is with 12 edge bridging hydrides. This gives a low potential energy minimum and there are no short H...H contacts. For 1a one of the hydrides $(\mathrm{H} 12 / \mathrm{H} 13)$ has been assigned as terminal split with 50\% occupancy over two sites, from HYDEX it models happily as a single edge bridging hydride. Figure S4 shows the structure with the 12 hydrides in calculated positions. Notable is that it is very similar to both the experimentally observed X-ray structures apart from the two half occupancy terminal hydrides in 1a. Attempts to fit 8 face capping hydrides and then the other four hydrides as terminal did not work. Placing the hydrides as 12 terminal hydrides results in the experimentally observed structure with 12 edge bridging hydrides.

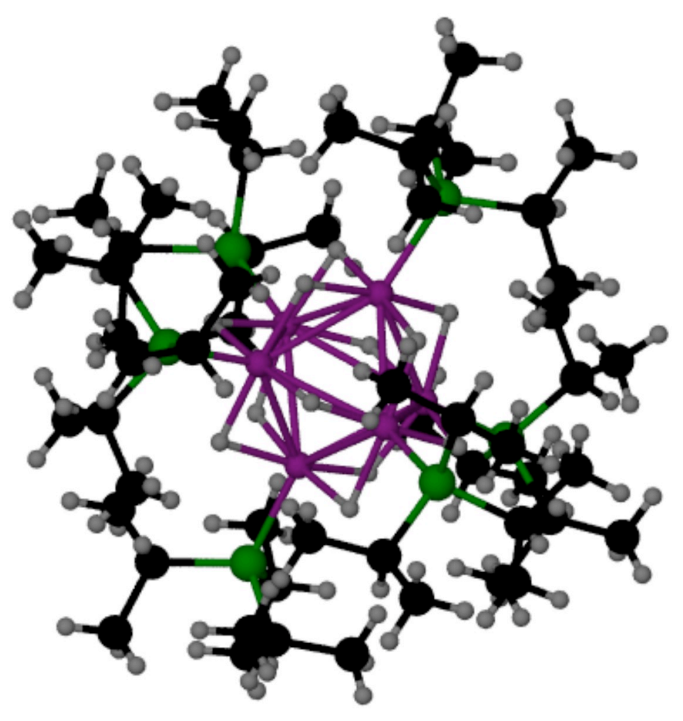

Figure S4. Result of HYDEX analysis of the twelve hydrides on clusters $\mathbf{1 a}$ and $\mathbf{1} \mathbf{b}$ showing the (experimentally also observed) 12 bridging hydride positions. 